\title{
Kinetic theory for financial Brownian motion from microscopic dynamics
}

\author{
Kiyoshi Kanazawa, ${ }^{1,2}$ Takumi Sueshige, ${ }^{2}$ Hideki Takayasu, ${ }^{1,3}$ and Misako Takayasu ${ }^{1,2}$ \\ ${ }^{1}$ Institute of Innovative Research, Tokyo Institute of Technology, 4259 Nagatsuta-cho, Midori-ku, Yokohama 226-8502, Japan \\ ${ }^{2}$ Department of Mathematical and Computing Science, School of Computing, Tokyo Institute of Technology, \\ 4259 Nagatsuta-cho, Midori-ku, Yokohama 226-8502, Japan \\ ${ }^{3}$ Sony Computer Science Laboratories, 3-14-13 Higashi-Gotanda, Shinagawa-ku, Tokyo 141-0022, Japan
}

(Received 21 March 2018; published 29 November 2018)

\begin{abstract}
Recent technological development has enabled researchers to study social phenomena in detail, and financial markets have attracted the attention of physicists particularly since key concepts in Brownian motion are applicable to the description of financial systems. In our previous Letter [Kanazawa et al., Phys. Rev. Lett. 120, 138301 (2018)], we presented a microscopic model of high-frequency traders (HFTs) through direct data analyses of individual trajectories of HFTs and revealed its theoretical dynamics by introducing the Boltzmann and Langevin equations for finance. However, the formulation therein was rather heuristic and a more mathematically exact derivation is necessary from the microscopic dynamics of the HFT model. We hereby establish the mathematical foundation of kinetic theory for financial Brownian motion in a manner parallel to traditional statistical physics. We first derive the exact time-evolution equation for the phase-space distribution for the HFT model, corresponding to the Liouville equation in analytical mechanics. By a systematic reduction of the Liouville equation for finance, the Bogoliubov-Born-Green-Kirkwood-Yvon hierarchal equations are generalized for financial Brownian motion. We then derive the Boltzmann and Langevin equations for the order book and the price dynamics by assuming molecular chaos. The asymptotic solutions to these equations are presented for a large number of HFTs, which qualitatively reveal how the strategies of traders at the microscopic level impact the macroscopic dynamics of market price. Our theoretical prediction was numerically examined via Monte Carlo simulations. Our kinetic description highlights the parallel mathematical structure between the financial and physical Brownian motions by a straightforward extension of statistical mechanics.
\end{abstract}

DOI: 10.1103/PhysRevE.98.052317

\section{INTRODUCTION}

The goal of statistical physics is to reveal macroscopic behavior of physical systems from their microscopic setups, and this has been partially achieved in equilibrium and nonequilibrium statistical mechanics [1]. For example, kinetic theory has provided a mathematically rigid foundation for various nonequilibrium systems such as dilute molecular gas, Brownian motion, granular gas, active matter, traffic flows, neural networks, and social dynamics [2-12]. The fundamental equations of kinetic theory (i.e., the Boltzmann and Langevin equations) were historically introduced on the basis of phenomenological arguments within the frameworks of nonlinear master equations and stochastic processes [13,14]. Furthermore, their systematic derivations were mathematically developed from analytical mechanics by Bogoliubov-Born-Green-KirkwoodYvon (BBGKY) and van Kampen [14-17].

Inspired by these successes, physicists have attempted to apply statistical physic approaches beyond material science to the social sciences. In particular, financial markets have

Published by the American Physical Society under the terms of the Creative Commons Attribution 4.0 International license. Further distribution of this work must maintain attribution to the author(s) and the published article's title, journal citation, and DOI. attracted the attention of physicists as an interdisciplinary field $[18,19]$ since they exhibit quite similar phenomena which can be described by key concepts in physics, such as Brownian motion. It is noteworthy that the concept of Brownian motion was historically first formulated for financial systems by Bachelier [20] before the famous work by Einstein in field physics [21]. Subsequent to Bachelier's studies, several characteristics of Brownian motions in finance and their differences from physical Brownian motions have been discovered by both theoretical and data analyses. On the level of price time series, the power-law behavior of price movements has been reported empirically [22-26]. Such universal characters have been summarized as the stylized facts [19] and have been theoretically investigated using time-series models [19,27-29] and agent-based models [30-37]. In addition, the characteristics of order books (i.e., current distribution of quoted prices) have been studied using both empirical analysis and orderbook models [19,38-44]. For example, the zero-intelligence order-book models [38-44] have been investigated from several perspectives, such as power-law price movement statistics [38], order-book profile [41], and market impact by large meta orders $[43,44]$. The collective motion of the full order book was further found by analyzing the layered structure of the order book $[45,46]$, which was a key to the generalization of the fluctuation-dissipation relation to financial Brownian motion. To date, however, the modeling of individual traders dynamics based on direct microscopic evidence has not been 
fully studied, which is a crucial obstacle in the application of statistical mechanics from microscopic dynamics. To fully apply statistical mechanics to financial systems, it is necessary to establish a microscopic dynamical model of traders based on microscopic evidence, and to develop a nonequilibrium statistical mechanics for such non-Hamiltonian many-body systems.

Recently, an extension of the kinetic framework for financial Brownian motion was proposed by studying highfrequency data including traders identifiers (IDs) [46]. The dynamics of high-frequency traders (HFTs) were directly analyzed by tracking the trajectories of individuals, and a microscopic model of trend-following HFTs was established showing agreement with empirical analyses of microscopic trajectories. On the basis of the "equation of motions" for the HFTs, Boltzmann-like and Langevin-like equations were finally derived for mesoscopic and macroscopic dynamics, respectively. This framework is shown to be consistent with empirical findings, such as HFTs' trend following, average order-book profile, price movement, and layered order-book structure. However, the mathematical argument therein was rather heuristic, similarly to the original derivation of the conventional Boltzmann and Langevin equations. Considering the traditional stream of kinetic theory, a mathematical derivation beyond heuristics is necessary for the financial Brownian motion parallel to the works by BBGKY and van Kampen.

In this paper, we examine the mathematical foundation for financial Brownian motion in the parallel mathematics of kinetic theory. For the trend-following HFT model [46], we first define the phase space and the corresponding phasespace distribution (PSD) according to analytical mechanics $[15,47]$. We then derive the exact time-evolution equation for the PSD, which corresponds to the Liouville equation in analytical mechanics. The many-body dynamics for the PSD are reduced into few-body dynamics for reduced PSD according to the reduction method by BBGKY. By assuming molecular chaos, we obtain the nonlinear Boltzmann equation for the order-book profile and the master Boltzmann equation for the market price dynamics. We also present their perturbative solutions for a large number of HFTs to study the dynamic behavior of this system for all hierarchies. The validity of our framework is finally examined by Monte Carlo simulation.

This paper is organized as follows: In Sec. II, we briefly review the mathematical structure of the standard kinetic theory and the double-auction systems for price formulation before proceeding to our work. In Sec. III, we describe the details of the trend-following HFTs model as the microscopic setup. In Sec. IV, the microscopic dynamics of the model are exactly formulated in terms of the Liouville equation and the corresponding BBGKY hierarchal equations. In Sec. V, the financial Boltzmann equation is derived as the mesoscopic description of the financial system. In Sec. VI, the macroscopic behavior is analyzed by deriving the financial Langevin equation. In Sec. VII, implications of our theory are discussed for several related topics. We conclude this paper in Sec. VIII with some remarks. In this paper, the presentation of the main results is written as self-contained, followed by the detailed derivation of the results; readers interested only in the main results may skip their theoretical derivation without referring to the detailed calculation.

\section{BRIEF REVIEW OF CONVENTIONAL KINETIC THEORY FOR BROWNIAN MOTION AND FINANCIAL MARKET MICROSTRUCTURE}

Before proceeding to the core part of our work, we briefly review (i) the formulation of the kinetic theory for physical Brownian motion and (ii) the price formulation mechanism in financial markets via the double-auction systems, to convey our essential idea for generalization toward financial systems. The aim of this section is to provide sufficient background knowledge for nonexperts in kinetic theory or financial market microstructure. Since our work is based on interdisciplinary techniques in statistical physics and financial market microstructure, we believe that these reviews will help familiarize readers to these fields, although the main results in this paper are self-contained.

\section{A. Review of kinetic theories from the equation of motions}

We first start with a review of kinetic theory from microscopic Newton dynamics. Let us consider the Hamiltonian dynamics of $N$ gas particles of mass $m$ and a tracer particle of mass $M$ with the hard-core interaction in a hard-core box of volume $V$ [see Fig. 1(a) for a schematic]. The momentum and position of the $i$ th gas particle are denoted by $\boldsymbol{p}_{i} \equiv$ $\left(p_{i ; x}, p_{i ; y}, p_{i ; z}\right)$ and $\boldsymbol{q}_{i} \equiv\left(q_{i ; x}, q_{i ; y}, q_{i ; z}\right)$ for $1 \leqslant i \leqslant N$, and those of the tracer are denoted by $\boldsymbol{P}=\boldsymbol{p}_{0}$ and $\boldsymbol{Q}=\boldsymbol{q}_{0}$. The dynamics of this system are described by the equation of motions,

$$
\frac{d \boldsymbol{q}_{i}}{d t}=\frac{\boldsymbol{p}_{i}}{m_{i}}, \quad \frac{d \boldsymbol{p}_{i}}{d t}=\sum_{j \neq i} \boldsymbol{F}_{i j},
$$

with interaction force $\boldsymbol{F}_{i j}$ between particles $i$ and $j$ for $0 \leqslant$ $i, j \leqslant N\left(m_{i}=M\right.$ for $i=0$ and $m_{i}=m$ otherwise $)$.

\section{B. Liouville equation}

In analytical mechanics, the phase space is defined as $\mathcal{S} \equiv$ $\prod_{i=0}^{N}(-\infty, \infty)^{6}$. The state of a system can be designated as the phase point defined by $\boldsymbol{\Gamma} \equiv\left(\boldsymbol{P}, \boldsymbol{Q} ; \boldsymbol{p}_{1}, \boldsymbol{q}_{1} ; \ldots ; \boldsymbol{p}_{N}, \boldsymbol{q}_{N}\right) \in$ $\mathcal{S}$, and the corresponding PSD is denoted by $P_{t}(\boldsymbol{\Gamma})$. The time evolution of the PSD is described by the Liouville equation,

$$
\frac{\partial P_{t}(\boldsymbol{\Gamma})}{\partial t}=\mathcal{L} P_{t}(\boldsymbol{\Gamma})
$$

with the Liouville operator $\mathcal{L}^{1}$ (see Refs. [14,15,47-50] for the details). This equation is mathematically exactly equivalent to the equation of motions (1), and is the fundamental equation for the microscopic description [Fig. 1(a)]. However, the equation is not analytically solvable since it fully addresses the original many-body dynamics without any approximation.

\footnotetext{
${ }^{1}$ In the presence of the hard-core interaction, the Liouville operator $\mathcal{L}$ is nonlocal and is technically called the pseudo-Liouville operator $[14,48-50]$.
} 


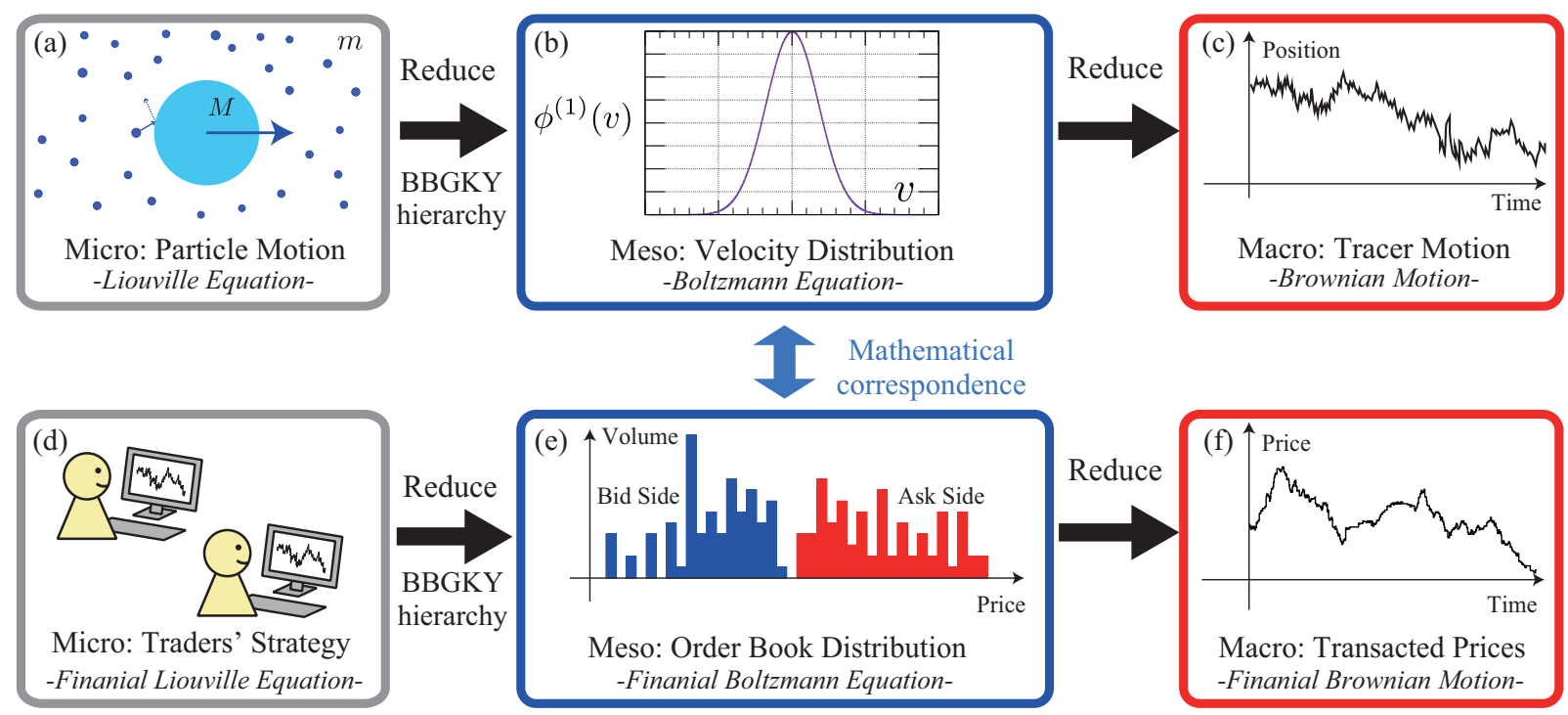

FIG. 1. Hierarchal kinetic description of the conventional Brownian motion (a)-(c) and the financial Brownian motion (d)-(f). In the microscopic hierarchy of physical Brownian motion (a), gas particles and a massive tracer interact with each other, where the dynamics are described by the Liouville equation (2). As the mesoscopic description (b), the full dynamics are reduced to the one-body distribution $\phi^{(1)}$ for gas particles, which are governed by the Boltzmann equation (6). The macroscopic dynamics of the tracer (c) are described by the master Boltzmann equation (8), or the Langevin equation (9) asymptotically for large system size $M \gg m$. Here we focus on the parallel hierarchal structure of financial markets (d)-(f) to molecular kinetic theory. In the microscopic hierarchy (d), each trader makes decisions to submit or cancel orders. As such, the dynamics of the traders correspond to those of molecules in kinetic theory. In the mesoscopic hierarchy (e), the information on trader identifiers is lost by coarse graining and we obtain the dynamics of the order book (i.e., the quoted price distribution). The order-book profile corresponds to the velocity distribution in the conventional kinetic theory. In the macroscopic hierarchy (f), the dynamics of the market price movement are finally deduced by the coarse graining, which exhibits anomalous random walks. The market price dynamics correspond to those of the Brownian motion in kinetic theory.

\section{BBGKY hierarchy and Boltzmann equation}

To focus on the one-body dynamics of a gas particle or the tracer, let us introduce the reduced PSDs,

$$
\begin{aligned}
\phi_{t}^{(1)}\left(\boldsymbol{p}_{1}, \boldsymbol{q}_{1}\right) & \equiv \int P_{t}(\boldsymbol{\Gamma}) \prod_{i=0, i \geqslant 2} d \boldsymbol{p}_{i} d \boldsymbol{q}_{i}, \\
\phi_{t}^{(2)}\left(\boldsymbol{p}_{1}, \boldsymbol{q}_{1}, \boldsymbol{p}_{2}, \boldsymbol{q}_{2}\right) & \equiv \int P_{t}(\boldsymbol{\Gamma}) \prod_{i=0, i \geqslant 3} d \boldsymbol{p}_{i} d \boldsymbol{q}_{i}, \\
P_{t}^{(\mathrm{T})}(\boldsymbol{P}, \boldsymbol{Q}) & \equiv \int P_{t}(\boldsymbol{\Gamma}) \prod_{i \geqslant 1} d \boldsymbol{p}_{i} d \boldsymbol{q}_{i}, \\
P_{t}^{(\mathrm{TG})}\left(\boldsymbol{P}, \boldsymbol{Q}, \boldsymbol{p}_{1}, \boldsymbol{q}_{1}\right) & \equiv \int P_{t}(\boldsymbol{\Gamma}) \prod_{i \geqslant 2} d \boldsymbol{p}_{i} d \boldsymbol{q}_{i} .
\end{aligned}
$$

On the assumption of binary interaction, we can exactly derive hierarchies of the PSDs from the Liouville equation (2), such that

$$
\begin{aligned}
\frac{\partial \phi_{t}^{(1)}}{\partial t} & =\mathcal{L}^{(1)} \phi_{t}^{(1)}+\mathcal{L}^{(2)} \phi_{t}^{(2)}+\frac{1}{N} \mathcal{L}^{(\mathrm{TG})} P_{t}^{(\mathrm{TG})}, \\
\frac{\partial P_{t}^{(\mathrm{T})}}{\partial t} & =\mathcal{L}^{(\mathrm{T})} P_{t}^{(\mathrm{T})}+\mathcal{L}^{(\mathrm{TG})} P_{t}^{(\mathrm{TG})}
\end{aligned}
$$

with one-body Liouville operators $\mathcal{L}^{(1)}, \mathcal{L}^{(\mathrm{T})}$ and two-body collision operators $\mathcal{L}^{(2)}, \mathcal{L}^{(\mathrm{TG})}$. These equations are exact but not closed in terms of $\phi_{t}^{(1)}$ and $P_{t}^{(\mathrm{T})}$.
To obtain analytical solutions, a further approximation is necessary. The standard approximation in kinetic theory is a mean-field approximation, called molecular chaos,

$$
\phi^{(2)}\left(\boldsymbol{p}_{1}, \boldsymbol{q}_{1}, \boldsymbol{p}_{2}, \boldsymbol{q}_{2}\right) \approx \phi^{(1)}\left(\boldsymbol{p}_{1}, \boldsymbol{q}_{1}\right) \phi^{(1)}\left(\boldsymbol{p}_{2}, \boldsymbol{q}_{2}\right),
$$

which is mathematically shown to be asymptotically exact for dilute gas in the thermodynamic limit $N, V \rightarrow \infty$ (called the Boltzmann-Grad limit [51]). We then obtain the closed dynamical equation for $\phi^{(1)}$ as

$$
\frac{\partial \phi^{(1)}}{\partial t} \approx \mathcal{L}^{(1)} \phi^{(1)}+\mathcal{L}^{(2)}\left(\phi^{(1)} \phi^{(1)}\right)
$$

which is the fundamental equation for the mesoscopic description [Fig. 1(b)]. The steady solution for $\phi^{(1)}$ of the nonlinear Boltzmann equation (6) is then given by the celebrated Maxwell-Boltzmann distribution.

\section{Langevin equation}

The stochastic dynamics for the macroscopic variables $(\boldsymbol{P}, \boldsymbol{Q})$ can also be obtained within kinetic theory. By applying molecular chaos for $P^{(\mathrm{TG})}\left(\boldsymbol{P}, \boldsymbol{Q}, \boldsymbol{p}_{1}, \boldsymbol{q}_{1}\right)$ as

$$
P^{(\mathrm{TG})}\left(\boldsymbol{P}, \boldsymbol{Q}, \boldsymbol{p}_{1}, \boldsymbol{q}_{1}\right) \approx P^{(\mathrm{T})}(\boldsymbol{P}, \boldsymbol{Q}) \phi^{(1)}\left(\boldsymbol{p}_{1}, \boldsymbol{q}_{1}\right),
$$

we obtain the master Boltzmann equation (or the linear Boltzmann equation)

$$
\frac{\partial P^{(\mathrm{T})}}{\partial t} \approx \mathcal{L}^{(\mathrm{T})} P^{(\mathrm{T})}+\mathcal{L}^{(\mathrm{TG})}\left(P^{(\mathrm{T})} \phi^{(1)}\right),
$$


(a)



(b) Volume

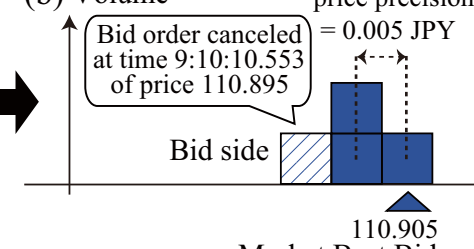

Market Best Bid
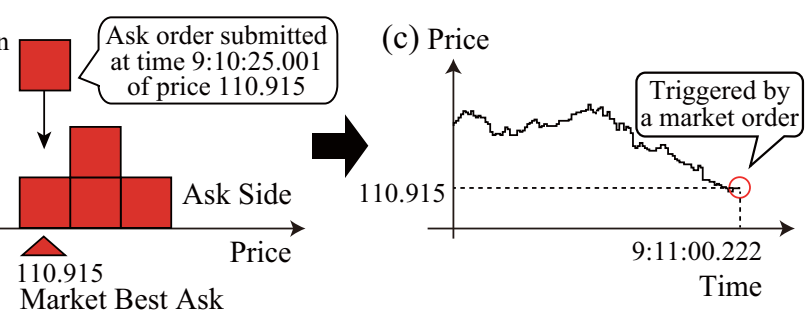

FIG. 2. Schematic of the price formulation via the double-auction systems. (a) Individual traders make decisions on limit, market, and cancellation orders. In this schematic, the first trader canceled his/her bid quote of price 110.895 JPY at 9:10:10:10.553; the second trader submitted his/her ask limit order of price 110.915 JPY at 9:10:25.001; the third trader submitted a buy market order at 9:11:00.222. (b) Cancellation and limit orders are reflected in the order book as depletion and injection of "blocks." Here the blocks represent orders of the unit volume (i.e., one million dollars) and are allocated on the lattice with minimum interval 0.005 JPY. (c) A new market price is generated when prices match, as an interaction between limit and market orders.

which belongs to the linear-master equations in the Markov process and describes the dynamics of the tracer particle. Equation (8) can be further approximated as the FokkerPlanck equation within the system size expansion, which was developed by van Kampen [16]. For the macroscopic limit $M \gg m$, one can asymptotically deduce the Langevin equation for the tracer as the macroscopic description of Brownian motion [Fig. 1(c)],

$$
\frac{d \boldsymbol{P}}{d t} \approx-\frac{\gamma}{M} \boldsymbol{P}+\sqrt{2 \gamma T} \boldsymbol{\xi}^{\mathrm{G}},
$$

with viscous coefficient $\gamma$, temperature of the gas $T$, and white Gaussian noise $\xi^{\mathrm{G}}$ with unit variance.

The above formulation shows the systematic connection from the microscopic Newtonian dynamics to the mesoscopic dynamics and macroscopic dynamics. This methodology is shown to be valid even for nonequilibrium systems when the gas is sufficiently dilute (see Refs. [3-9,12] for its application to various nonequilibrium systems), and is one of the most successful formulations in statistical physics.

\section{E. Review of price formation mechanism in the double-auction financial markets}

The aforementioned kinetic theory is generalized for financial markets in this paper. Let us then review the price formation mechanism in financial markets via the doubleauction systems, which is a popular framework adopted in many financial markets, such as foreign exchange (FX) and stock markets. This subsection is aimed to provide sufficient background knowledge to statistical physicists who are not familiar with financial markets.

Here we introduce five technical terms related to the price formulation in the double-auction systems: limit order, order book, market order, cancellation order, and liquidity.

(1) Limit order. In the double-auction markets, traders submit limit orders to show their quoted prices, the prices at which they are willing to transact [Fig. 2(a)]. There are two types of quoted prices: bid and ask. A bid (ask) quoted price means the price at which they are willing to buy (sell) the currency. A limit order also accompanies volume, the total amount of currencies that the trader is willing to transact. We note that both price and volume are typically discretized; for example, the minimum price precision was 0.005 yen and the minimum volume unit was one million dollars in our interbank market data provided by Electronic Broking Services (EBS) on the exchange between the US dollar (USD) and Japanese yen (JPY) (see Sec. III B for the detailed data description). In the following, we measure volume using the volume minimum unit. For example, a bid (ask) limit order of price 110.905 JPY with a unit volume implies that the trader is willing to buy (sell) one million USD by paying (receiving) $110.905 \times 10^{6}$ JPY.

(2) Order book. Quoted prices reside in the order book, the current distribution of bid and ask quoted orders [see Fig. 2(b) for a schematic]. When a trader submits an ask limit order, for example, the order will enter and reside in the ask order book until it is transacted or canceled. Here the horizontal axis represents the price and the vertical axis represents the total volumes of orders at the price in Fig. 2(b). We note that the price axis is discretized by the minimum price precision like a one-dimensional lattice system. The order book is the key element to the double-auction systems since it provides the current information on the demands and supplies for the currency. The highest bid and lowest ask quoted prices are called the market best bid and ask prices, respectively.

(3) Market order. A transaction occurs when bid and ask prices match between different traders: traders exchange their currency pair at the market transacted price (or the market price for short). Transactions can be triggered by market orders, ${ }^{2}$ orders to immediately buy (sell) the currency at the market best ask (bid) prices, and the market price is reflected in the price time series [Fig. 2(c)]. A market order has a gender, buy or sell.

(4) Cancelation order. Traders can cancel their limit orders by cancellation orders [Fig. 2(b)]. In particular, HFTs frequently cancel their limit orders and update their prices, in order to take advantage of their response speed. Not-canceled limit orders are called live orders to stress their difference from canceled limit orders.

(5) Liquidity. The market liquidity implies the degree to which the currency can be quickly bought or sold without any

\footnotetext{
${ }^{2}$ Technically, an ask (bid) limit order of lower (higher) price than the market best bid (ask) price can immediately trigger a transaction, akin to a market order. The difference between limit orders and market orders is subtle in this sense.
} 
price impact. The price impact of a market order tends to be significant in the cases of a lack of bid or ask limit orders and the double-auction markets of plenty bid and ask orders are regarded as liquid markets. Maintenance of sufficient numbers of orders for both bid and ask sides is highly appreciated and there are specialists that provide such market liquidity, called liquidity providers, as discussed later.

The aforementioned mechanisms are implemented in the double-auction systems for price formulation, where the price time series is not generated by pure random numbers but is formed as part of the psychological battles and implicit negotiations among traders through both order book and price time series.

To capture such complex structures, there are several models for price formulation dynamics that focus on various viewpoints. For example, one of the popular approaches is to ignore the details of the order-book dynamics, and to focus on economical perspectives (e.g., the information asymmetry among traders in Kyle's classical model [30]). While this approach highlights fundamental economical properties, various properties of the order book are missed, such as the book profile and its relaxation dynamics. Another approach is to directly model the order-book dynamics based on only simple assumptions, to avoid introduction of ad hoc parameters. For example, the zero-intelligence models are based on simple Poisson processes for the order submission, cancellation, and execution [38-44]. This approach also highlights the basic characteristics of the order-book dynamics under minimal assumptions. In addition, these approaches have the advantages that the model calibration is easy under coarse-grained data sets; they require only the price time series or order book for calibration. Indeed, the availability of precise microscopic data sets was limited, which had made it difficult to scientifically model the trader dynamics on the level of individuals.

Recently, however, ultimately microscopic data including trader IDs have become available, which has made it scientifically possible to directly model the details of individual trader dynamics. In Ref. [46], a microscopic model of individual trader dynamics was introduced as a stochastic multiagent model by utilizing the trading log data on the level of traders. The authors found a novel empirical law on the trend-following behavior of traders, which characterizes the collective motion of individual traders and was missing in the previous order-book models. We here stress that the microscopic data were critically helpful in constructing the microscopic model without introduction of ad hoc parameters. The developed model was finally shown to be consistent with the mesoscopic and macroscopic findings from the data set.

Since the microscopic model has been established by a real data analysis, it is necessary to reveal the model dynamics by developing a corresponding statistical mechanical theory. The aim of this paper is to develop a statistical mechanical theory for such a financial Brownian motion model based on molecular kinetic theory.

\section{F. Idea to generalize kinetic theory toward finance}

Herein, we will present our ideas with regard to generalizing the framework toward a financial Brownian motion. Financial markets have a quite similar hierarchal structure to conventional Brownian motion [see Figs. 1(d)-1(f) for a schematic]: In the microscopic hierarchy, individual traders make decisions to buy or sell currencies at a certain price [Fig. 1(d)]. In the mesoscopic hierarchy, the dynamics are coarse-grained into the order-book dynamics with the removal of traders' IDs [Fig. 1(e)]. In the macroscopic hierarchy, the dynamics are reduced to the price dynamics [Fig. 1(f)]. One can notice that these hierarchies directly correspond to those in kinetic theory; traders, order book, and price correspond to molecules, velocity distribution, and Brownian particle, respectively. In this sense, the financial markets have a similar hierarchal structure to that in kinetic theory. From the next section, we present a parallel mathematical framework for the description of financial markets from microscopic dynamics.

\section{MICROSCOPIC SETUP}

In this section, the dynamics of the trend-following HFT model in Ref. [46] is mathematically formulated within the many-body stochastic processes with collisions on the basis of microscopic empirical evidence.

\section{A. Notation}

We here briefly explain the notation used in this paper. Any stochastic variable accompanies the hat symbol such as $\hat{A}$ to stress its difference from nonstochastic real numbers such as $A$. For example, the probability distribution function (PDF) of a stochastic variable $\hat{A}(t)$ at real time $t$ is denoted by $P(A, t) \equiv P(\hat{A}(t)=A)$ with a nonstochastic real number $A$ [i.e., the probability of $\hat{A}(t) \in[A, A+$ $d A)$ is given by $P(A, t) d A]$. The complementary cumulative distribution function (CDF) is also defined as $P(\geqslant$ $A, t) \equiv \int_{A}^{\infty} P\left(A^{\prime}, t\right) d A^{\prime}$. Arguments in functions are abbreviated without mention sometimes to simplify the notation. The ensemble average of any stochastic quantity $\hat{A}(t)$ is denoted by $\langle\hat{A}(t)\rangle \equiv \int_{-\infty}^{\infty} A P(A, t) d A$.

We next explain the terminology for the order book for the whole market [Fig. 3(a)]. The highest bid (lowest ask) quoted price among all the traders is called the market best bid (ask) price $\hat{b}_{M}\left(\hat{a}_{M}\right)$. The average of the market best bid and ask prices is called the market midprice $\hat{z}_{M} \equiv\left(\hat{b}_{M}+\hat{a}_{M}\right) / 2$. The difference between the market best bid and ask prices $\hat{L}_{M} \equiv$ $\hat{a}_{M}-\hat{b}_{M}$ is called the market spread. The market transacted price refers to the price at which a transaction occurs in the market. In this paper, the market price (mathematically denoted by $\hat{p}$ ) indicates the market transacted price for short.

For a single trader, the highest bid (lowest ask) quoted price by a single trader is called the best bid (ask) price of the trader in this paper, which is denoted by $\hat{b}_{i}\left(\hat{a}_{i}\right)$ for the $i$ th trader [see Fig. 3(b)]. The average of the best bid and ask prices of the trader is called the midprice of the trader (denoted by $\hat{z}_{i}$ ). Also, the difference between the best bid and ask prices of the trader is called the buy-sell spread of the trader (denoted by $\hat{L}_{i} \equiv \hat{a}_{i}-\hat{b}_{i}$ ), which is conceptually different from the market spread $\hat{L}_{M}$.

There are two types of time used in this analysis. One is the real time $t$ and the other is the tick time $T$ [Fig. 3(c)]. The tick time $T$ is defined as a discrete time incremented by every market transaction and corresponds to the real time as a stochastic variable, such as $t=\hat{t}[T]$. Here the square 



FIG. 3. Notation of the state variables for our market model. (a) Market best bid $\hat{b}_{M}$ and ask $\hat{a}_{M}$ prices. The market midprice and market spread are defined by $\hat{z}_{M} \equiv\left(\hat{b}_{M}+\hat{a}_{M}\right) / 2$ and $\hat{L}_{M} \equiv \hat{a}_{M}-\hat{b}_{M}$, respectively. (b) Best bid $\hat{b}_{i}$ and ask $\hat{a}_{i}$ prices for a single trader $i$. Here the light blocks represent the orders submitted by the $i$ th trader. The midprice and buy-sell spread of the $i$ th trader are defined by $\hat{z}_{i} \equiv$ $\left(\hat{b}_{i}+\hat{a}_{i}\right) / 2$ and $\hat{L}_{i} \equiv \hat{a}_{i}-\hat{b}_{i}$, respectively. (c) Schematic of the tick time $T$, incremented every transaction. For the trend-following analysis of individual traders [46], the correlation was studied between future movement of HFT's quoted midprice $\Delta \hat{z}_{i}[T]$ and historical price movement $\Delta \hat{p}[T-1]$.

brackets for the function's argument (e.g., $\hat{A}[T])$ imply that the stochastic variable $\hat{A}(t)$ is measured according to the tick time $T$ [i.e., $\hat{A}[T] \equiv \hat{A}(\hat{t}[T])]$, highlighting the difference from that measured according to the real time $t$ [e.g., $\hat{A}(t)$ with round brackets].

\section{B. Data description and characters of real HFTs}

Here we describe the characters of real HFTs on the basis of high-frequency data analysis of an interbank FX market. We analyzed the order-book data including anonymized trader IDs and anonymized bank codes on EBS from the 5th 18:00 to the 10th 22:00 GMT June 2016. ${ }^{3}$ EBS is an interbank FX market and is one of the biggest financial platforms in the world. The minimum volume unit for transaction was one million USD for the FX market between the USD and the JPY. Since the EBS market is an interbank platform, most of the market participants are professional institutional investors; they are typically associated with banks (or hedge funds via the EBS prime dealing service). Our FX data set is therefore essentially the trading log data of institutional investors but not of retail investors; most of the traders can be assumed to have professional skills of FX trading.

For this study, we focus particularly on HFTs, who frequently submit or cancel their orders according to algorithms. As reported in our previous work [46], HFTs have several characteristics that are quite different from those of lowfrequency traders (LFTs). In this paper, an HFT is defined as a trader who submitted limit orders more than 2500 times during the week (i.e., at least approximately one submission every minute on average, assuming that their working time is eight hours per day). Here, we acknowledge that there are a variety of definitions of HFTs in both econophysics and finance but we use the simplest definition based on the number of order submissions during the week, similarly to previous research [52]. With this definition, the number of HFTs was 135 during the week, while the total number of traders submitting limit orders was $922,{ }^{4}$ and $89.6 \%$ of all the orders in this market were submitted by the HFTs. Here we

\footnotetext{
${ }^{3}$ We purchased this data set for academic use only from NEX Group plc, a third-party for-profit company.

${ }^{4}$ In Ref. [46] 134 traders were defined as HFTs with one trader excluded whose order lifetime is extremely short.
}

summarize the reported characteristics with several additional pieces of evidence:

( $\alpha 1)$ Small number of live orders and volume. HFTs typically maintain a few live orders, less than ten [see Figs. 4(a) and 4(b)]. Furthermore, a single order submitted by HFTs typically implies one unit volume of the currency. These characteristics are in contrast to those of LFTs, who sometimes submit large volumes in a single order [see Figs. 4(a) and 4 (c) for the fat-tailed distributions of the number of orders or volumes for LFTs].

$(\alpha 2)$ Liquidity providers. Typical HFTs play the role of key liquidity providers and have the obligation to maintain continuous two-way quotes during their liquidity hours according to the EBS rulebook [53] [see Fig. 4(d) for a typical trajectory of the top HFT]. Here the two-way quotes implies that the trader quotes both bid and ask prices to provide liquidity for the market. The balance between the ask and bid order book is kept statistically symmetric to some extent, seemingly thanks to the liquidity providers.

$(\alpha 3)$ Frequent price modification. Typical HFTs frequently modify their quoted prices by successive submission and cancellation of orders [see Fig. 4(d) for a typical trajectory of the top HFT]. The lifetime of orders was typically within seconds for the top HFT, while the typical transaction interval was 9.3 seconds in our data set. In addition, $94.4 \%$ of the submissions by all the HFTs were canceled without transactions.

$(\alpha 4)$ Trend-following property. HFTs tend to follow the market trends. We here denote the best bid and ask quoted price of the $i$ th trader and the market price at the $T$ tick time by $\hat{b}_{i}(\hat{t}[T]) \equiv \hat{b}_{i}[T], \hat{a}_{i}(\hat{t}[T]) \equiv \hat{a}_{i}[T]$, and $\hat{p}(\hat{t}[T]) \equiv$ $\hat{p}[T]$, respectively [see Fig. 3(c)]. We also denote the mid quoted price of the $i$ the trader by $\hat{z}_{i}[T] \equiv\left(\hat{b}_{i}[T]+\hat{a}_{i}[T]\right) / 2$. According to Ref. [46], the future price movement of the $i$ th HFT $\Delta \hat{z}_{i}[T] \equiv \hat{z}[T+1]-\hat{z}[T]$ statistically obeys

$$
\left\langle\Delta \hat{z}_{i}[T]\right\rangle_{\Delta \hat{p}[T-1]=\Delta p} \approx c_{i} \tanh \frac{\Delta p}{\Delta p_{i}^{*}}
$$

conditionally on the historical price movement $\Delta \hat{p}[T-1] \equiv$ $\hat{p}[T]-\hat{p}[T-1]=\Delta p$ with the characteristic constants $c_{i}$ and $\Delta p_{i}^{*}$. The constant $c_{i}$ characterizes the strength of trend following for the $i$ th trader, whereas $\Delta p_{i}^{*}$ characterizes the saturation threshold for the trader's reaction to market trends. Here the ensemble average $\langle\ldots\rangle_{\Delta \hat{p}[T-1]=\Delta p}$ is taken for active traders $\Delta \hat{z}_{i}[T] \neq 0$ on the condition that the previous price movement is given by $\Delta \hat{p}[T-1]=\Delta p$ with a 


$$
\text { (a) HFTs }
$$

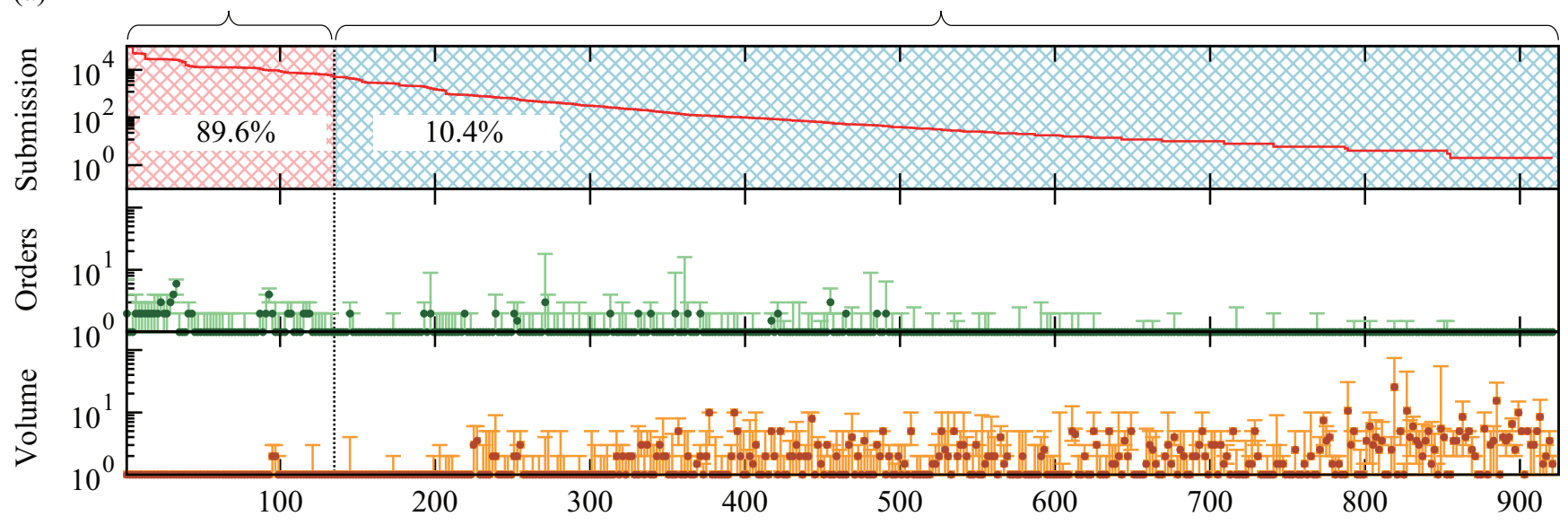

Trader ranking sorted by the number of the total submissions
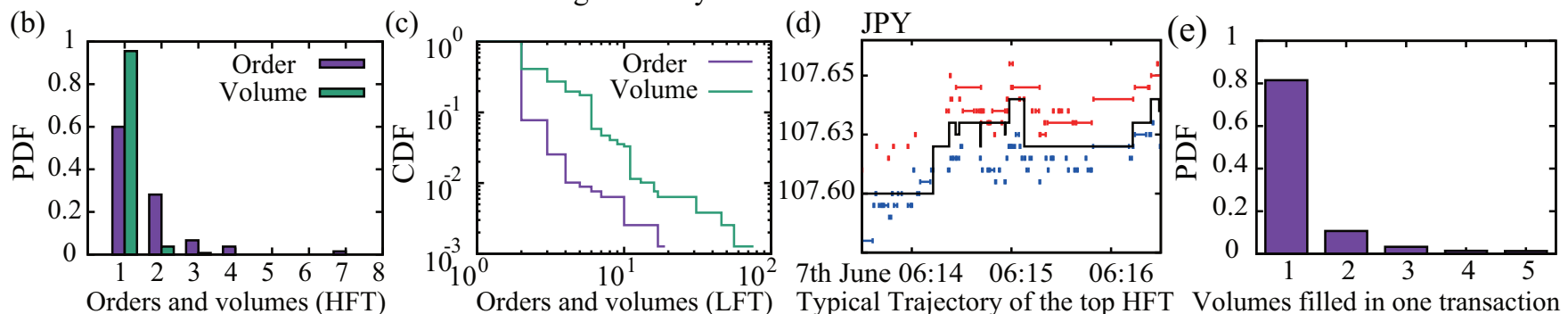

FIG. 4. Summary of the empirical characters of real HFTs based on our data set. (a) The number of submissions, typical number of orders, typical volumes designated in one order, depending on the ranking of the trader. For this figure, we analyzed representative numbers for every two traders for anonymization. Top: We sorted the traders by their total number of submissions to define their rankings. The top 135 traders were defined as HFTs, while the remaining 788 traders were defined as LFTs in this paper. We plotted the average of their total submissions for every two traders. Center: We investigated the number of total orders in the bid (ask) side at every bid (ask) order submission and take its median, first, and third quartiles every two traders. Bottom: We studied the volumes designated in one order at every order submission and take its median, first, and third quartiles for every two traders. (b) PDF for the numbers of orders maintained by a single HFT for one side (purple) and volumes designated in a single order of HFTs (green). For this figure, we studied medians as representative numbers for every single HFT. (c) CDF for the numbers of orders maintained for one side (purple) by a single LFT and the volumes designated in a single order of LFTs (green). For this figure, we studied medians as representative numbers for every single LFT. (d) Typical trajectories of the top HFT, continuously maintaining both sides as key liquidity providers. (e) PDF for volumes filled in a single transaction. The percentage of one-to-one transaction is $81.5 \%$ of all transactions. Transactions within 5 volumes occupy $98.2 \%$.

nonstochastic real number $\Delta p$. In the following, we introduce shorthand notation for the conditional ensemble average as $\langle\ldots\rangle_{\Delta \hat{p}[T-1]=\Delta p}=\langle\ldots\rangle_{\Delta p}$. In addition, the variance of the HFT's future price movement is independent of historical market trends as

$$
V_{\Delta p}\left(\Delta \hat{z}_{i}[T]\right) \approx \sigma_{i}^{2}
$$

with variance $V_{\Delta p}\left(\Delta \hat{z}_{i}[T]\right) \equiv\left\langle\left(\Delta \hat{z}_{i}[T]-\left\langle\Delta \hat{z}_{i}[T]\right\rangle_{\Delta p}\right)^{2}\right\rangle_{\Delta p}$ and constant $\sigma_{i}^{2}$ independent of $\Delta p$.

We also note that the one-to-one transaction is the basic interaction among traders in this market. The percentage of one-to-one transactions was $81.5 \%$ in our data set [see Fig. 4(e) for more detailed evidence]. On the basis of the previous empirical results, the trend-following HFT model was proposed in Ref. [46] as the corresponding minimal microscopic model as reviewed in the next section.

\section{Theoretical model}

On the basis of the previously outlined characteristics of HFTs, let us consider a microscopic model according to
Ref. [46], within the framework of many-body stochastic systems with collisions.

\section{State variables}

Let us consider a market composed of $N$ HFTs quoting both bid and ask prices $\left\{\hat{b}_{i}\right\}_{i}$ and $\left\{\hat{a}_{i}\right\}_{i}$ at all time with the unit volume, where the index $i$ identifies the individual trader $(1 \leqslant i \leqslant N)$. This assumption is consistent with the empirical HFT's characteristics $(\alpha 1)$ and $(\alpha 2)$. For simplicity, the difference between the best bid and ask prices of a single trader (called buy-sell spread $L_{i}$ ) is assumed to be a time constant unique to the trader [see Fig. 5(a)]:

$$
L_{i} \equiv \hat{b}_{i}-\hat{a}_{i}=\text { const. }
$$

On this assumption, the dynamics of individual HFTs are uniquely characterized by their midprice as $\hat{z}_{i} \equiv\left(\hat{b}_{i}+\hat{a}_{i}\right) / 2$. The maximum and minimum values of the buy-sell spread among traders are denoted by $L_{\max }$ and $L_{\min }$, respectively. According to Ref. [46], the buy-sell distribution $\rho_{L}$ is directly 
(a) Volume

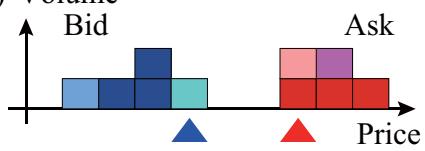

Market best bid Market best ask (b)



Market price (updated as $\hat{p}[T]$ )



$\left(L_{i}+L_{j}\right) / 2$

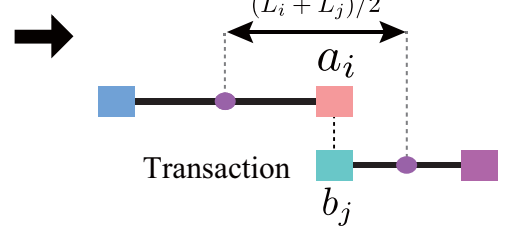

(c)
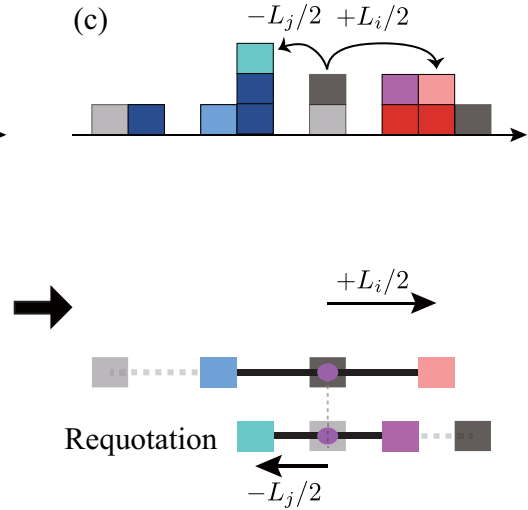

FIG. 5. Schematics of the dynamics of the trend-following HFT model. (a) Traders maintain two-sided quotes with constant buy-sell spreads $L_{i}$ and $L_{j}$ for traders $i$ and $j$. Their midprices move according to a deterministic trend following and random movement. (b) When bid and ask prices coincide, transaction occurs at that price. (c) Both traders requote their bid and ask prices at a distance from the market price after transaction.

measured to obey the $\gamma$ distribution, such that

$$
\rho_{L} \equiv \frac{1}{N} \sum_{i=1}^{N} \delta\left(L-L_{i}\right) \approx \frac{L^{\alpha}}{\alpha ! L^{*(\alpha+1)}} e^{-L / L^{*}}
$$

with decay length $L^{*}$ and empirical exponent $\alpha \approx 3$.

\section{Trend-following random walks}

HFTs have a tendency to maintain continuous two-sided quotes by frequently modifying their prices (i.e., successive cancellation and submission of limit orders), as required by the market rule [53]. This implies that the midprice trajectory of an HFT can be modeled as a continuous random trajectory [i.e., the characteristics $(\alpha 2)$ and ( $\alpha 3)$ ]. Remarkably, there is a mathematical theorem which guarantees that the Itô processes [i.e., stochastic differential equations (SDEs) driven by the white Gaussian noise] are the only Markov processes with a continuous sample trajectory [13]. As a minimal model satisfying all the characters of real HFTs $(\alpha 1)-(\alpha 4)$, the dynamics of the HFTs are modeled within the Itô processes as

$$
\frac{d \hat{z}_{i}}{d t}=c \tanh \frac{\Delta \hat{p}}{\Delta p^{*}}+\sigma \hat{\eta}_{i}^{\mathrm{R}}
$$

in the absence of transactions [Fig. 5(a)]. Here $c$ and $\Delta p^{*}$ are constants that characterize the strength and threshold of the trend-following effect and $\hat{\eta}_{i}^{\mathrm{R}}$ is the white Gaussian noise with unit variance. The presence of the trend-following effect in Eq. (14) is the characteristic of our HFT model, which induces the collective translational motion of full order book [46] and is crucial in reproducing the order-book layered structure reported in Ref. [45] (see Sec. III F).

\section{Transaction rule}

When the best bid and ask prices coincide, a transaction occurs [see Fig. 5(b)]. The transaction condition (i.e., the condition of price matching) is mathematically given by

$$
\hat{b}_{j}=\hat{a}_{i}
$$

for $i \neq j$. In the following, we assume that the index $i$ is an integer different from another integer $j$. At the instance of the transaction $\hat{b}_{j}=\hat{a}_{i}$, let us assume that the traders requote their prices simultaneously [see Fig. 5(c)] such that

$$
\hat{b}_{j}^{\mathrm{pst}}=\hat{b}_{j}-\frac{L_{j}}{2}, \quad \hat{a}_{i}^{\mathrm{pst}}=\hat{a}_{i}+\frac{L_{i}}{2},
$$

where $\hat{b}_{j}^{\mathrm{pst}}$ and $\hat{a}_{i}^{\mathrm{pst}}$ are post-transactional bid and ask prices, respectively. By introducing the midprice of the individual traders as $\hat{z}_{i} \equiv\left(\hat{b}_{i}+\hat{a}_{i}\right) / 2$, the transaction rule is rewritten as

$$
\hat{z}_{i}-\hat{z}_{j}=\frac{L_{i}+L_{j}}{2} \Longrightarrow \hat{z}_{i}^{\mathrm{pst}}=\hat{z}_{i}-\frac{L_{i}}{2}, \quad \hat{z}_{j}^{\mathrm{pst}}=\hat{z}_{j}+\frac{L_{j}}{2} .
$$

We hereby define the market price $\hat{p}(t)$ and the previous price movement $\Delta \hat{p}(t)$ at time $t . \hat{p}(t)$ is the market price at the previous transaction; $\Delta \hat{p}(t)$ is the price movement by the previous transaction. They are updated after transactions under the following post-transaction rule [Figs. 5(b) and 5(c)]:

$$
\begin{aligned}
\left|\hat{z}_{i}-\hat{z}_{j}\right| & =\frac{L_{i}+L_{j}}{2} \Longrightarrow \hat{p}^{\mathrm{pst}}=\hat{z}_{i}-\frac{L_{i}}{2} \operatorname{sgn}\left(\hat{z}_{i}-\hat{z}_{j}\right), \\
\Delta \hat{p}^{\mathrm{pst}} & =\hat{z}_{i}-\frac{L_{i}}{2} \operatorname{sgn}\left(\hat{z}_{i}-\hat{z}_{j}\right)-\hat{p},
\end{aligned}
$$

with signature function $\operatorname{sgn}(x)$ defined by $\operatorname{sgn}(x)=x /|x|$ for $x \neq 0$ and $\operatorname{sgn}(0)=0$.

\section{Complete model dynamics}

We hereby specify the complete dynamics of the quoted prices $\left\{\hat{z}_{i}(t)\right\}_{i}$ within the framework of stochastic processes with collisions. When the previous price movement is $\Delta \hat{p}$, we assume that the traders' quoted prices are described by trend-following random walks:

$$
\begin{aligned}
\frac{d \hat{z}_{i}}{d t} & =c \tanh \frac{\Delta \hat{p}}{\Delta p^{*}}+\sigma \hat{\eta}_{i}^{\mathrm{R}}+\hat{\eta}_{i}^{\mathrm{T}}, \\
\hat{\eta}_{i}^{\mathrm{T}} & \equiv \sum_{k=1}^{\infty} \sum_{j} \Delta \hat{z}_{i j} \delta\left(t-\hat{\tau}_{k ; i j}\right),
\end{aligned}
$$


TABLE I. Summary of the model parameters and their dimensions.

\begin{tabular}{lll}
\hline \hline Parameter & \multicolumn{1}{c}{ Meaning } & \multicolumn{1}{c}{ Dimension } \\
\hline$N$ & Number of traders & dimensionless \\
$\left\{L_{i}\right\}_{1 \leqslant i \leqslant N}$ & Buy-sell spreads of traders & price \\
$c$ & Strength of trend following & price/time \\
$\Delta p^{*}$ & Saturation for trend following & price \\
$\sigma^{2}$ & Variance of random noise & price $^{2} /$ time \\
\hline \hline
\end{tabular}

where $\hat{\eta}_{i}^{\mathrm{T}}$ is a requotation jump term and $\hat{\tau}_{k ; i j}$ is the $k$ th transaction time between traders $i$ and $j$ satisfying

$$
\begin{aligned}
\left|\hat{z}_{i}\left(\hat{\tau}_{k ; i j}\right)-\hat{z}_{j}\left(\hat{\tau}_{k ; i j}\right)\right| & =\frac{L_{i}+L_{j}}{2}, \\
\Delta \hat{z}_{i j} & \equiv-\frac{L_{i}}{2} \operatorname{sgn}\left(\hat{z}_{i}-\hat{z}_{j}\right) .
\end{aligned}
$$

The requotation jump $\hat{\eta}_{i}^{\mathrm{T}}$ corresponds to collisions in molecular kinetic theory. The price-matching condition and the requotation rule (17) correspond to the contact condition and the momentum exchange rule in standard kinetic theory for hard-sphere gases, respectively. The summary of the model parameters is presented in Table I with their dimensions. A sample trajectory of this model is depicted in Fig. 6(a). This model is a generalization of the previous theoretical model in Refs. [31,34-37] on the basis of the aforementioned empirical facts $(\alpha 1)-(\alpha 4)$ on HFTs.

The dynamics of the price $\hat{p}$ and the previous price movement $\Delta \hat{p}$ can be specified within the framework of stochastic processes. Since $\hat{p}$ and $\Delta \hat{p}$ are updated at the instance of the transactions, their dynamics synchronize with the collision time $\hat{\tau}_{k ; i j}$. Considering the transaction rule for prices (18), their concrete dynamical equations are thus given by

$$
\begin{gathered}
\frac{d \hat{p}}{d t}=\sum_{k=1}^{\infty} \sum_{i, j}^{i<j}\left(\hat{p}_{i j}^{\mathrm{pst}}-\hat{p}\right) \delta\left(t-\hat{\tau}_{k ; i j}\right), \\
\frac{d \Delta \hat{p}}{d t}=\sum_{k=1}^{\infty} \sum_{i, j}^{i<j}\left(\Delta \hat{p}_{i j}^{\mathrm{pst}}-\Delta \hat{p}\right) \delta\left(t-\hat{\tau}_{k ; i j}\right),
\end{gathered}
$$

with the price after collision $\hat{p}_{i j}^{\mathrm{pst}} \equiv \hat{z}_{i}-\left(L_{i} / 2\right) \operatorname{sgn}\left(\hat{z}_{i}-\hat{z}_{j}\right)$ and the price movement after collision $\Delta \hat{p}_{i j}^{\mathrm{pst}} \equiv \hat{p}_{i j}^{\mathrm{pst}}-\hat{p}$. In this paper, the Itô convention is used for the multiplication to $\delta$ functions.

\section{E. Slow variable}

Introduction of slow variables is key to reduction of complex dynamics in general (e.g., the center of mass (c.m.) of the Brownian particle [16] and the slaving principles in synergetics [54]). Here we introduce the c.m. of the quoted prices as the slow variable of this system [Fig. 6(a)]. The definition of the c.m. and its dynamics are given by

$$
\hat{z}_{\mathrm{c} . \mathrm{m} .} \equiv \frac{1}{N} \sum_{i=1}^{N} \hat{z}_{i}, \quad \frac{d \hat{z}_{\mathrm{c} . \mathrm{m} .}}{d t}=c \tanh \frac{\Delta \hat{p}}{\Delta p^{*}}+\bar{\eta},
$$

with $\bar{\eta} \equiv(\sigma / N) \sum_{i=1}^{N} \hat{\eta}_{i}^{\mathrm{R}}+(1 / N) \sum_{i=1}^{N} \hat{\eta}_{i}^{\mathrm{T}}$. The c.m. $\hat{z}_{\mathrm{c} . \mathrm{m}}$. characterizes the macroscopic dynamics of this system. As will be shown in Sec. VIC 1, the diffusion coefficient of the c.m. is proportional to $N^{-1}$ for the weak trend-following case, which implies that the selection of $\hat{z}_{\text {c.m. }}$ is reasonable as a slow variable.

Another motivation to introduce the c.m. is to define the relative price from the c.m. such that

$$
\hat{r}_{i} \equiv \hat{z}_{i}-\hat{z}_{\mathrm{c} . \mathrm{m} .},
$$

since the relative price $\hat{r}_{i}$ has better mathematical characteristics than $\hat{z}_{i}$. For example, the relative price $\hat{r}_{i}$ fluctuates around zero [see Fig. 6(b) for the dynamics in the comoving frame of c.m.] and has the stationary distribution, while the original variable $\hat{z}_{i}$ diffuses to infinity for a long time and has no stationary distribution.

\section{F. Difference from other order-book models}

One of the unique characters of the HFT model is the collective motion of the order book due to trend following. As in Ref. [45], the order book has the layered structure in the sense that the difference in volumes of bid (ask) order book near best price has positive (negative) correlation with price movements. This implies that the order book exhibits translational motion like inertia in physics [Fig. 6(c)], and thus movements of HFTs are not independent of each other like herding behavior. This collective motion has not been implemented in conventional zero-intelligence order-book models [38-44],
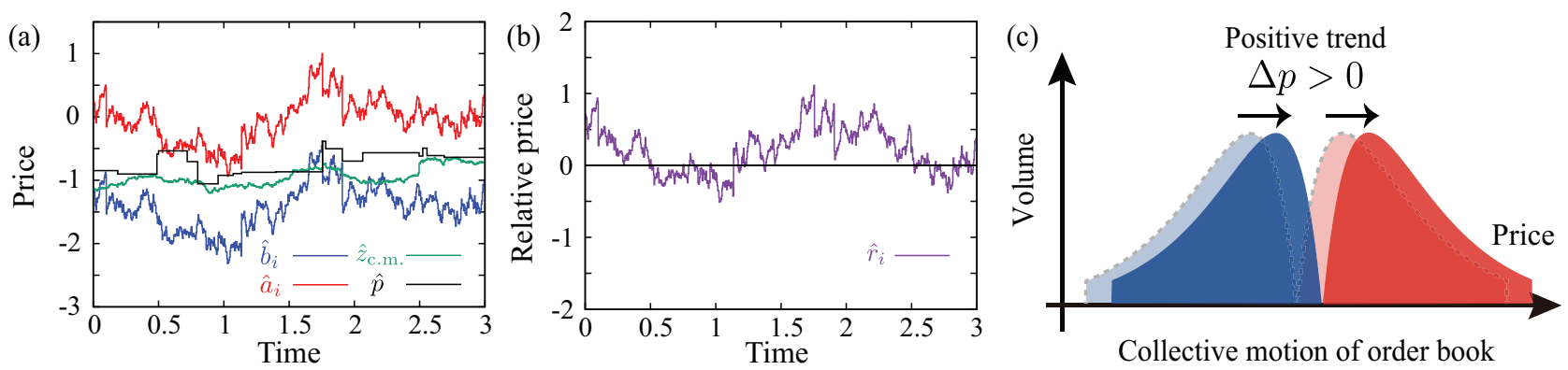

FIG. 6. Schematics of the microscopic and mesoscopic dynamics of the HFT model. (a) Sample trajectory of an HFT, showing the bid $\hat{b}_{i}$ and ask $\hat{a}_{i}$ quoted prices of the $i$ th trader, the c.m. $\hat{z}_{\text {c.m. }}$, and the market transaction price $\hat{p}$. (b) Sample trajectory of the relative price $\hat{r}_{i}$ from the c.m. $\hat{z}_{\text {c.m. }}$, showing that $\hat{r}_{i}$ stationarily fluctuates around zero. (c) Collective motion of the order book, showing herding behavior of traders. This collective motion is minimally implemented as trend following. 

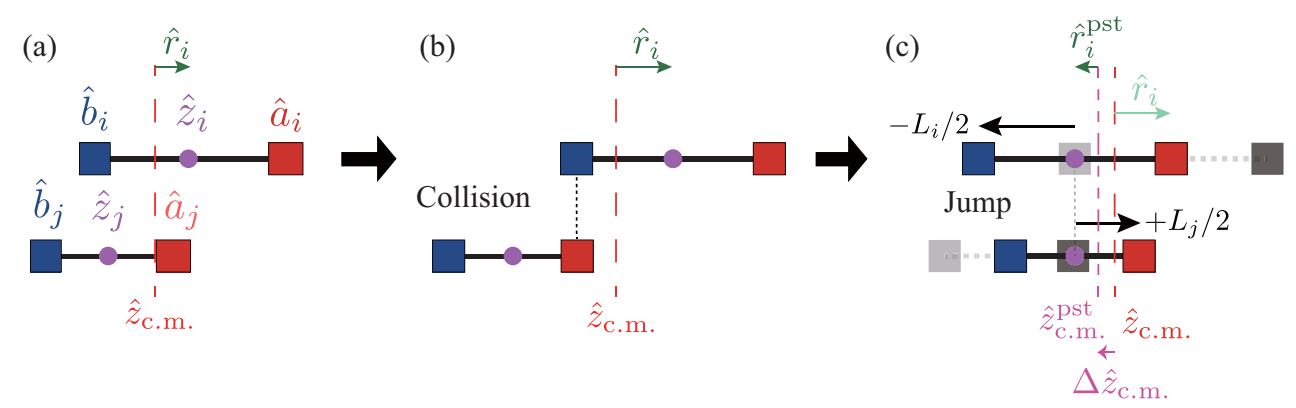

FIG. 7. Schematic of the two-body collision. When the prices match between the traders $i$ and $j$, they requote their prices far from the market price. Note that the c.m. also moves through a distance of $\Delta \hat{z}_{\mathrm{c} . \mathrm{m} .}=-\left(L_{i}-L_{j}\right) / 2 N$ during this requotation.

which are based on independent Poisson processes for order submission and cancellation, and is minimally implemented in our trend-following HFT model for consistency with the layered order-book structure [46].

\section{MAIN RESULT 1: MICROSCOPIC DESCRIPTION}

As the main results of this paper, the analytical solutions of the trend-following HFT model are presented by developing the mathematical techniques of kinetic theory. We first introduce the phase space for the HFT model in the standard manner of analytical mechanics, and derive the dynamical equation for the PSD, which we call the financial Liouville equation. We next derive the hierarchy for the reduced distributions similarly to the BBGKY hierarchy in molecular kinetic theory, which is the theoretical key to systematic understanding of the financial system as shown in Secs. V and VI.

\section{A. Phase space and phase-space distribution}

Here we first introduce the phase space for the HFT model according to the standard manner of analytical mechan- ics. Let us introduce a vector $\hat{\boldsymbol{\Gamma}} \equiv\left(\hat{z}_{1}, \ldots, \hat{z}_{N} ; \hat{z}_{\mathrm{c} . \mathrm{m} .}, \hat{p}, \Delta \hat{p}\right)$, which corresponds to a phase point in the phase space $\mathcal{S} \equiv \prod_{i=1}^{N+3}(-\infty, \infty)$ as $\hat{\boldsymbol{\Gamma}} \in \mathcal{S}$. Equations (19), (21), and (22) are the complete set of dynamical equations for the phase point, corresponding to the Newtonian equations of motions in conventional mechanics. Also, let us define the PSD function $P_{t}(\boldsymbol{\Gamma})$. Using the PSD, the probability where the phase point $\Gamma$ exists at the time $t$ in the volume element $d \boldsymbol{\Gamma} \equiv \prod_{i=1}^{N}\left[z_{i}, z_{i}+d z_{i}\right) \times\left[z_{\mathrm{c} . \mathrm{m} .}, z_{\mathrm{c} . \mathrm{m} .}+\right.$ $\left.d z_{\text {c.m. }}\right) \times[p, p+d p) \times[\Delta p, \Delta p+d \Delta p)$ as $\hat{\boldsymbol{\Gamma}} \in d \boldsymbol{\Gamma}$ is given by $P_{t}(\boldsymbol{\Gamma}) d \boldsymbol{\Gamma}$.

\section{B. Financial Liouville equation}

As the first main result in this paper, we present the Liouville equation for the trend-following trader model (19)(22) as the dynamical equation for the PSD. The dynamical equation for the PSD is given by

$$
\frac{\partial P_{t}(\boldsymbol{\Gamma})}{\partial t}=\mathcal{L}^{\mathrm{a}} P_{t}(\boldsymbol{\Gamma})+\mathcal{L}^{\mathrm{c}} P_{t}(\boldsymbol{\Gamma})
$$

where the advective and diffusive Liouville operator $\mathcal{L}^{\mathrm{a}}$ and the binary collision Liouville operator $\mathcal{L}^{\mathrm{c}}$ are defined by

$$
\begin{aligned}
\mathcal{L}^{\mathrm{a}} P_{t} & \equiv \sum_{i=1}^{N}\left[-c \tanh \frac{\Delta p}{\Delta p^{*}}\left\{\partial_{i}+\frac{1}{N} \partial_{\mathrm{c} . \mathrm{m} .}\right\}+\frac{\sigma^{2}}{2}\left\{\partial_{i}+\frac{1}{N} \partial_{\mathrm{c} . \mathrm{m} .}\right\}^{2}\right] P_{t}(\boldsymbol{\Gamma}), \\
\mathcal{L}^{\mathrm{c}} P_{t} & \equiv \sum_{i, j} \frac{\sigma^{2}}{2}\left\{\delta\left(z_{i}-z_{j}\right) \delta\left(p-z_{i}\right) \int d \Delta p^{\prime}\left|\tilde{\partial}_{i j}\right| P_{t}\left(\boldsymbol{\Gamma}-\Delta \boldsymbol{\Gamma}_{i j}^{\prime}\right)-\delta\left(z_{i}-z_{j}-\frac{L_{i}+L_{j}}{2}\right)\left|\tilde{\partial}_{i j}\right| P_{t}(\boldsymbol{\Gamma})\right\} .
\end{aligned}
$$

Here we have introduced the symmetric absolute derivative $\left|\tilde{\partial}_{i j}\right| f \equiv\left|\partial_{i} f\right|+\left|\partial_{j} f\right|$ for an arbitrary function $f\left(z_{i}, z_{j}\right)$ and abbreviated derivatives $\partial_{i} \equiv \partial / \partial z_{i}$ and $\partial_{\text {c.m. }} \equiv \partial / \partial z_{\text {c.m. }}$ (see the detailed derivation below). We have also introduced a difference vector representing the two-body collision (see Fig. 7),

$$
\Delta \boldsymbol{\Gamma}_{i j}^{\prime} \equiv\left(0, \ldots,-\frac{L_{i}}{2}, \ldots,+\frac{L_{j}}{2}, \ldots, 0 ; \Delta z_{\mathrm{c} . \mathrm{m} .}, \Delta p, \Delta p-\Delta p^{\prime}\right),
$$

with movement of the c.m. $\Delta z_{\text {c.m. }} \equiv-\left(L_{i}-L_{j}\right) / 2 N$. This is the first main result of this paper. The advective and diffusive Liouville operator $\mathcal{L}^{\text {a }}$ describes the continuous dynamics of the system in the absence of transactions, while the binary collision Liouville operator $\mathcal{L}^{c}$ describes the discontinuous dynamics in the presence of transactions. Equation (24) formally corresponds to the Liouville equation (2) in molecular kinetic theory, and is called the financial Liouville equation in this paper. The financial Liouville equation completely characterizes the microscopic dynamics of all traders [Fig. 1(d)]. 


\section{Derivation}

The financial Liouville equation for the trader model (19) is derived as follows. Let us formulate our model by

$$
\frac{d \hat{z}_{i}}{d t}=c \tanh \frac{\Delta p}{\Delta p^{*}}+\sigma \hat{\eta}_{i ; \varepsilon}^{\mathrm{R}}+\hat{\eta}_{i}^{\mathrm{T}}
$$

with a colored Gaussian noise $\hat{\eta}_{i ; \varepsilon}^{\mathrm{R}}$ satisfying $\left\langle\hat{\eta}_{i ; \varepsilon}^{\mathrm{R}}\right\rangle=0$ and $\left\langle\hat{\eta}_{i ; \varepsilon}^{\mathrm{R}}(t) \hat{\eta}_{i ; \varepsilon}^{\mathrm{R}}(s)\right\rangle=e^{-|t-s| / \varepsilon} / 2 \varepsilon$. For mathematical convenience, we finally take the white noise limit $\varepsilon \rightarrow+0: \lim _{\varepsilon \rightarrow 0} \hat{\eta}_{i ; \varepsilon}^{\mathrm{R}}=\hat{\eta}_{i}^{\mathrm{R}}$. The dynamics of the c.m. $\hat{z}_{\mathrm{c} . \mathrm{m} .}$ are given by

$$
\frac{d \hat{z}_{\mathrm{c} . \mathrm{m} .}}{d t}=c \tanh \frac{\Delta p}{\Delta p^{*}}+\bar{\eta}, \quad \bar{\eta} \equiv \frac{\sigma}{N} \sum_{i=1}^{N} \hat{\eta}_{i ; \varepsilon}^{\mathrm{R}}+\frac{1}{N} \sum_{i, j}^{i<j} \sum_{k=1}^{\infty}\left(\Delta \hat{z}_{i j}+\Delta \hat{z}_{j i}\right) \delta\left(t-\hat{\tau}_{k ; i j}\right) .
$$

We also consider the dynamics of an arbitrary function $f(\hat{\boldsymbol{\Gamma}})$ for $\hat{\boldsymbol{\Gamma}} \equiv\left(\hat{z}_{1}, \ldots, \hat{z}_{N} ; \hat{z}_{\mathrm{c} . \mathrm{m} .}, \hat{p}, \Delta \hat{p}\right) \in \mathcal{S}$. The time evolution of $f(\hat{\boldsymbol{\Gamma}})$ is governed by the continuous movement by the continuous noise term $\hat{\eta}_{i ; \varepsilon}^{\mathrm{R}}$ and the discontinuous jumps by the deterministic transaction term $\hat{\eta}_{i}^{\mathrm{T}}$. We then obtain

$$
\begin{aligned}
\frac{d f(\hat{\boldsymbol{\Gamma}})}{d t}= & \sum_{i=1}^{N} \frac{\partial f}{\partial \hat{r}_{i}}\left\{c \tanh \frac{\Delta \hat{p}}{\Delta p^{*}}+\sigma \hat{\eta}_{i ; \varepsilon}^{\mathrm{R}}\right\}+\frac{\partial f}{\partial \hat{z}_{\mathrm{c} . \mathrm{m} .}}\left\{c \tanh \frac{\Delta \hat{p}}{\Delta p^{*}}+\frac{\sigma}{N} \sum_{i=1}^{N} \hat{\eta}_{i ; \varepsilon}^{\mathrm{R}}\right\} \\
& +\sum_{k=1}^{\infty} \sum_{i, j}^{i<j}\left[f\left(\hat{\boldsymbol{\Gamma}}+\Delta \hat{\boldsymbol{\Gamma}}_{i j}\right)-f(\hat{\boldsymbol{\Gamma}})\right] \delta\left(t-\hat{\tau}_{k ; i j}\right),
\end{aligned}
$$

where we have introduced the difference vector $\Delta \hat{\boldsymbol{\Gamma}}_{i j}$ induced by transactions defined by

$$
\Delta \hat{\boldsymbol{\Gamma}}_{i j} \equiv(0, \ldots, 0, \overbrace{-\frac{L_{i}}{2} \operatorname{sgn}\left(\hat{z}_{i}-\hat{z}_{j}\right)}^{i \text { th }}, 0, \ldots, 0, \overbrace{-\frac{L_{j}}{2} \operatorname{sgn}\left(\hat{z}_{j}-\hat{z}_{i}\right)}^{j \text { th }}, 0, \ldots, 0 ;-\frac{L_{i}-L_{j}}{2 N} \operatorname{sgn}\left(\hat{z}_{i}-\hat{z}_{j}\right), \hat{p}_{i j}^{\mathrm{pst}}-\hat{p}, \Delta \hat{p}_{i j}^{\mathrm{pst}}-\Delta \hat{p})
$$

with $\hat{p}_{i j}^{\mathrm{pst}} \equiv \hat{z}_{i}-\left(L_{i} / 2\right) \operatorname{sgn}\left(\hat{z}_{i}-\hat{z}_{j}\right)$ and $\Delta p_{i j}^{\mathrm{pst}} \equiv \hat{p}_{i j}^{\mathrm{pst}}-\hat{p}$. Let us decompose the sum of $\delta$ functions as

$$
\begin{aligned}
\sum_{i, j}^{i<j} \sum_{k=1}^{\infty} \delta\left(t-\hat{\tau}_{k ; i j}\right) & =\sum_{i, j}^{i<j}\left[\sigma \delta\left(\hat{z}_{i}-\hat{z}_{j}-\frac{L_{i}+L_{j}}{2}\right)\left(\hat{\eta}_{i ; \varepsilon}^{\mathrm{R}}-\hat{\eta}_{j ; \varepsilon}^{\mathrm{R}}\right)-\sigma \delta\left(\hat{z}_{i}-\hat{z}_{j}+\frac{L_{i}+L_{j}}{2}\right)\left(\hat{\eta}_{i ; \varepsilon}^{\mathrm{R}}-\hat{\eta}_{j ; \varepsilon}^{\mathrm{R}}\right)\right] \\
& =\sum_{i, j} \sigma \delta\left(\hat{z}_{i}-\hat{z}_{j}-\frac{L_{i}+L_{j}}{2}\right)\left(\hat{\eta}_{i ; \varepsilon}^{\mathrm{R}}-\hat{\eta}_{j ; \varepsilon}^{\mathrm{R}}\right)
\end{aligned}
$$

where we have used $\hat{\eta}_{i ; \varepsilon}^{\mathrm{R}}-\hat{\eta}_{j ; \varepsilon}^{\mathrm{R}}>0$ just before $\hat{z}_{i}-\hat{z}_{j}-\left(L_{i}+L_{j}\right) / 2=0$ [or equivalently, $\hat{\eta}_{i ; \varepsilon}^{\mathrm{R}}-\hat{\eta}_{j ; \varepsilon}^{\mathrm{R}}<0$ just before $\hat{z}_{i}-\hat{z}_{j}+$ $\left(L_{i}+L_{j}\right) / 2=0$ ] by taking collision directions into account. We then take the ensemble average of both sides of Eq. (29) with the aid of Novikov's theorem [59] for an arbitrary functional $g\left[\hat{\eta}_{i ; \varepsilon}^{\mathrm{R}}\right]$,

$$
\left\langle\hat{\eta}_{i ; \varepsilon}^{\mathrm{R}}(t) g\left[\hat{\eta}_{i ; \varepsilon}^{\mathrm{R}}\right]\right\rangle=\int_{0}^{t} d s\left\langle\hat{\eta}_{i ; \varepsilon}^{\mathrm{R}}(t) \hat{\eta}_{i ; \varepsilon}^{\mathrm{R}}(s)\right\rangle\left\langle\frac{\delta g\left[\eta_{i ; \varepsilon}^{\mathrm{R}}\right]}{\delta \eta_{i ; \varepsilon}^{\mathrm{R}}(s)}\right\rangle
$$

for the colored Gaussian noise $\hat{\eta}_{i ; \varepsilon}^{\mathrm{R}}$. Here we note the following two important relations for the $\delta$ function for the phase space $\delta(\hat{\boldsymbol{\Gamma}}-\boldsymbol{\Gamma}) \equiv \delta\left(\hat{z}_{\mathrm{c.m} .}-z_{\mathrm{c} . \mathrm{m} .}\right) \delta(\hat{p}-p) \delta(\Delta \hat{p}-\Delta p) \prod_{i=1}^{N} \delta\left(\hat{z}_{i}-z_{i}\right)$ :

$$
\begin{aligned}
\lim _{\varepsilon \rightarrow 0}\left\langle\delta(\hat{\boldsymbol{\Gamma}}-\boldsymbol{\Gamma}) \delta\left(\hat{z}_{i}-\hat{z}_{j}-\left(L_{i}+L_{j}\right) / 2\right) \hat{\eta}_{i ; \varepsilon}^{\mathrm{R}}\right\rangle & =\delta\left(z_{i}-z_{j}-\left(L_{i}+L_{j}\right) / 2\right) \lim _{\varepsilon \rightarrow 0}\left\langle\delta(\hat{\boldsymbol{\Gamma}}-\boldsymbol{\Gamma}) \hat{\eta}_{i ; \varepsilon}^{\mathrm{R}}\right\rangle \\
& =\frac{\sigma}{2} \delta\left(z_{i}-z_{j}-\left(L_{i}+L_{j}\right) / 2\right)\left\langle\left[\frac{\partial}{\partial \hat{z}_{i}}+\frac{1}{N} \frac{\partial}{\partial \hat{z}_{\mathrm{c} . \mathrm{m} .}}\right] \delta(\hat{\boldsymbol{\Gamma}}-\boldsymbol{\Gamma})\right\rangle \\
& =-\frac{\sigma}{2} \delta\left(z_{i}-z_{j}-\left(L_{i}+L_{j}\right) / 2\right)\left[\partial_{i}+\frac{1}{N} \partial_{\mathrm{c} . \mathrm{m} .}\right] P_{t}(\boldsymbol{\Gamma})
\end{aligned}
$$


and

$$
\begin{aligned}
\lim _{\varepsilon \rightarrow 0}\left\langle\delta\left(\hat{\boldsymbol{\Gamma}}+\Delta \hat{\boldsymbol{\Gamma}}_{i j}-\boldsymbol{\Gamma}\right) \delta\left(\hat{z}_{i}-\hat{z}_{j}-\left(L_{i}+L_{j}\right) / 2\right) \hat{\eta}_{i ; \varepsilon}^{\mathrm{R}}\right\rangle & =\delta\left(z_{i}-z_{j}\right) \lim _{\varepsilon \rightarrow 0}\left\langle\delta\left(\hat{\boldsymbol{\Gamma}}+\Delta \hat{\boldsymbol{\Gamma}}_{i j}-\boldsymbol{\Gamma}\right) \hat{\eta}_{i ; \varepsilon}^{\mathrm{R}}\right\rangle \\
& =-\frac{\sigma}{2} \delta\left(z_{i}-z_{j}\right) \delta\left(p-z_{i}\right)\left[\partial_{i}+\frac{1}{N} \partial_{\mathrm{c} . \mathrm{m} .}\right] \int d \Delta p^{\prime} P_{t}\left(\boldsymbol{\Gamma}-\Delta \boldsymbol{\Gamma}_{i j}^{\prime}\right)
\end{aligned}
$$

with the dummy variable

$$
\Delta \boldsymbol{\Gamma}_{i j}^{\prime} \equiv\left(0, \ldots,-\frac{L_{i}}{2}, \ldots,+\frac{L_{j}}{2}, \ldots, 0 ;-\frac{L_{i}-L_{j}}{2 N}, \Delta p, \Delta p-\Delta p^{\prime}\right) .
$$

By substituting $f(\hat{\boldsymbol{\Gamma}})=\delta(\hat{\boldsymbol{\Gamma}}-\boldsymbol{\Gamma})$, we take the ensemble average for both sides of Eq. (29) in the $\varepsilon \rightarrow 0$ limit. We then obtain

$$
\begin{aligned}
\frac{\partial P_{t}(\boldsymbol{\Gamma})}{\partial t}= & \sum_{i=1}^{N}\left[-c \tanh \frac{\Delta p}{\Delta p^{*}}\left\{\partial_{i}+\frac{1}{N} \partial_{\text {c.m. }}\right\}+\frac{\sigma^{2}}{2}\left\{\partial_{i}+\frac{1}{N} \partial_{\text {c.m. }}\right\}^{2}\right] P_{t}(\boldsymbol{\Gamma}) \\
& +\sum_{i, j} \frac{\sigma^{2}}{2}\left\{-\delta\left(z_{i}-z_{j}\right) \delta\left(p-z_{i}\right) \int d \Delta p^{\prime} \tilde{\partial}_{i j} P_{t}\left(\boldsymbol{\Gamma}-\Delta \boldsymbol{\Gamma}_{i j}^{\prime}\right)+\delta\left(z_{i}-z_{j}-\frac{L_{i}+L_{j}}{2}\right) \tilde{\partial}_{i j} P_{t}(\boldsymbol{\Gamma})\right\}
\end{aligned}
$$

with an abbreviation symbol $\tilde{\partial}_{i j} \equiv \partial_{i}-\partial_{j}$. Here, we pay attention to the signature of the derivatives. Considering $P(\boldsymbol{\Gamma}) \geqslant 0$ for all $\boldsymbol{\Gamma}$ and $P(\boldsymbol{\Gamma})=0$ for $z_{i}-z_{j}>\left(L_{i}+L_{j}\right) / 2$, we obtain the signature of the derivatives

$$
\left.\partial_{i} P_{t}(\boldsymbol{\Gamma})\right|_{z_{i}-z_{j}=\left(L_{i}+L_{j}\right) / 2} \leqslant 0,\left.\quad \partial_{j} P_{t}(\boldsymbol{\Gamma})\right|_{z_{i}-z_{j}=\left(L_{i}+L_{j}\right) / 2} \geqslant 0 .
$$

Equation (36) can be simplified into Eq. (24) in terms of signatures by introducing the symmetric absolute derivative

$$
\left|\tilde{\partial}_{i j}\right| P_{t}(\boldsymbol{\Gamma}) \equiv\left|\partial_{i} P_{t}(\boldsymbol{\Gamma})\right|+\left|\partial_{j} P_{t}(\boldsymbol{\Gamma})\right| .
$$

Note that Eq. (24) is a partial integro-differential equation because of the transaction jumps. ${ }^{5}$

\section{Financial BBGKY hierarchy}

The financial Liouville equation (24) is exact but cannot be solved analytically. We therefore reduce Eq. (24) to a simplified dynamical equation for a one-body distribution in the parallel method to molecular kinetic theory. According to the standard method in kinetic theory, the Boltzmann equation, a closed dynamical equation for the one-body distribution, can be derived by systematically reducing the Liouville equation using the BBGKY hierarchy (see Sec. II C). We here present the lowest-order equation of reduced distributions for the trend-following HFT model in the parallel calculation to kinetic theory. We define the one-body, two-body, and three-body reduced distribution functions for the relative price $r_{i} \equiv z_{i}-z_{\text {c.m. }}$ :

$$
\begin{aligned}
P_{t}^{i}\left(r_{i}\right) & \equiv \int P_{t}(\boldsymbol{\Gamma}) d z_{\mathrm{c} . \mathrm{m} .} d p d \Delta p \prod_{l \neq i}^{N} d r_{l}, \quad P_{t}^{i j}\left(r_{i}, r_{j}\right) \equiv \int P_{t}(\boldsymbol{\Gamma}) d z_{\mathrm{c} . \mathrm{m} .} d p d \Delta p \prod_{l \neq i, j}^{N} d r_{l}, \\
P_{t}^{i j k}\left(r_{i}, r_{j}, r_{k}\right) & \equiv \int P_{t}(\boldsymbol{\Gamma}) d z_{\mathrm{c} . \mathrm{m} .} d p d \Delta p \prod_{l \neq i, j, k}^{N} d r_{l} .
\end{aligned}
$$

We then obtain the lowest-order hierarchal equation for the one-body distribution as

$$
\frac{\partial P_{t}^{i}\left(r_{i}\right)}{\partial t}=\mathcal{L}^{(i)} P_{t}^{i}\left(r_{i}\right)+\sum_{j \neq i} \mathcal{L}^{(i j)} P_{t}^{i j}\left(r_{i}, r_{j}\right)+\sum_{j, k \neq i} \mathcal{L}^{(i j k)} P_{t}^{i j k}\left(r_{i}, r_{j}, r_{k}\right)
$$

with one-body, two-body, and three-body Liouville operators $\mathcal{L}^{(i)}, \mathcal{L}^{(i j)}, \mathcal{L}^{(i j k)}$ defined by

$$
\begin{aligned}
\mathcal{L}^{(i)} P_{t}^{i} & \equiv \frac{\tilde{\sigma}^{2}}{2} \frac{\partial^{2} P_{t}^{i}\left(r_{i}\right)}{\partial r_{i}^{2}}, \\
\mathcal{L}^{(i j)} P_{t}^{i j} & \equiv \sum_{s= \pm 1} \frac{\sigma^{2}}{2}\left[\left.\left|\tilde{\partial}_{i j}\right| P_{t}^{i j}\left(r_{i}-\Delta r_{i j ; s}, r_{j}+\Delta r_{j i ; s}\right)\right|_{r_{i}=r_{j}}-\left.\left|\tilde{\partial}_{i j}\right| P_{t}^{i j}\left(r_{i}, r_{j}\right)\right|_{r_{i}-r_{j}=s\left(L_{i}+L_{j}\right) / 2}\right], \\
\mathcal{L}^{(i j k)} P_{t}^{i j} & \left.\equiv \sum_{s= \pm 1} \frac{\sigma^{2}}{2} \int d r_{j}\left[\left|\tilde{\partial}_{j k}\right| P_{t}^{i j k}\left(r_{i}-\Delta r_{j k ; s}^{(1)}, r_{j}, r_{k}\right)-\left|\tilde{\partial}_{j k}\right| P_{t}^{i j k}\left(r_{i}, r_{j}, r_{k}\right)\right]\right|_{r_{j}-r_{k}=s\left(L_{j}+L_{k}\right) / 2},
\end{aligned}
$$

\footnotetext{
${ }^{5}$ This implies that our financial Liouville equation (24) technically corresponds to the pseudo-Liouville equation [14,48-50] for hard-core interaction.
} 
effective variance $\tilde{\sigma}^{2} \equiv \sigma^{2}(1-1 / N)$, and jump size $\Delta r_{i j ; s} \equiv \Delta r_{i j ; s}^{(0)}+\Delta r_{i j ; s}^{(1)}$ with

$$
\Delta r_{i j ; s}^{(0)} \equiv-\frac{s L_{i}}{2}, \quad \Delta r_{i j ; s}^{(1)} \equiv \frac{s\left(L_{i}-L_{j}\right)}{2 N}
$$

Here $\Delta r_{i j ; s}^{(1)}$ indirectly originates from the movement of the c.m. during requotation. Equation (40) formally corresponds to the conventional BBGKY hierarchal equation (4a) for the mesoscopic description. On the basis of Eq. (40), the Boltzmann-type closed equation for the one-body distribution is derived in the next section.

We also derive the hierarchal equation for the macroscopic dynamics. For the macroscopic variables $Z \equiv\left(z_{\mathrm{c} . \mathrm{m}}, p, \Delta p\right)$, we define the reduced distributions:

$$
P_{t}(\boldsymbol{Z}) \equiv P_{t}\left(z_{\mathrm{c} . \mathrm{m} .}, p, \Delta p\right) \equiv \int P_{t}(\boldsymbol{\Gamma}) \prod_{k=1}^{N} d z_{k}, \quad P_{t}^{i j}\left(z_{i}, z_{j} ; \boldsymbol{Z}\right) \equiv P_{t}^{i j}\left(z_{i}, z_{j} ; z_{\mathrm{c} . \mathrm{m} .}, p, \Delta p\right) \equiv \int P_{t}(\boldsymbol{\Gamma}) \prod_{k \neq i, j}^{N} d z_{k}
$$

We then obtain the hierarchal equation for the macroscopic dynamics,

$$
\frac{\partial P_{t}(\boldsymbol{Z})}{\partial t}=\mathcal{L}_{\mathrm{c} . \mathrm{m} .}^{\mathrm{a}} P_{t}(\boldsymbol{Z})+\sum_{i, j} \mathcal{L}_{\mathrm{c} . \mathrm{m} .}^{\mathrm{c} i j} P_{t}^{i j}\left(z_{i}, z_{j} ; \boldsymbol{Z}\right)
$$

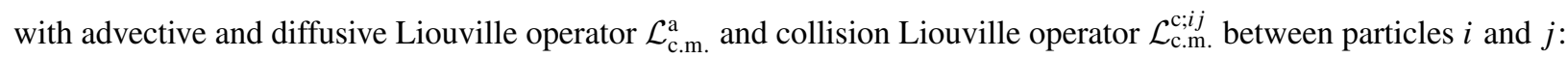

$$
\begin{aligned}
& \mathcal{L}_{\mathrm{c} . \mathrm{m} .}^{\mathrm{a}} P_{t}=\left[-c \tanh \frac{\Delta p}{\Delta p^{*}} \partial_{\mathrm{c} . \mathrm{m} .}+\frac{\sigma^{2}}{2 N} \partial_{\mathrm{c} . \mathrm{m} .}^{2}\right] P_{t}\left(z_{\mathrm{c} . \mathrm{m} .}, p, \Delta p\right), \\
& \mathcal{L}_{\mathrm{c} . \mathrm{m} .}^{\mathrm{c} i j} P_{t}=\frac{\sigma^{2}}{2}\left[\left.\int\left|\tilde{\partial}_{i j}\right| P_{t}^{i j}\left(z_{i}+\frac{L_{i}}{2}, z_{j}-\frac{L_{j}}{2} ; z_{\mathrm{c} . \mathrm{m} .}+\frac{L_{i}-L_{j}}{2 N}, p-\Delta p, \Delta p^{\prime}\right)\right|_{z_{i}=z_{j}=p} d \Delta p^{\prime}-\left.\int\left|\tilde{\partial}_{i j}\right| P_{t}^{i j}\right|_{z_{i}=z_{j}+\left(L_{i}+L_{j}\right) / 2} d z_{j}\right] .
\end{aligned}
$$

Equation (44) formally corresponds to the lowest-order conventional BBGKY hierarchal equation (8) for the macroscopic description. Using this hierarchal equation (44), a closed master Boltzmann equation is derived for the macroscopic variables in the next section.

The set of Eqs. (40) and (44) is the second main result in this paper. Equation (40) connects the microscopic description [Fig. 1(d)] to the mesoscopic description [Fig. 1(e)], and Eq. (44) connects the mesoscopic description [Fig. 1(e)] to the macroscopic description [Fig. 1(f)]. These equations are derived in a parallel calculation to the conventional BBGKY hierarchal equations (4), and are called the financial BBGKY hierarchal equations in this paper. Similarly to the conventional BBGKY hierarchal equations (4), our hierarchal equations (40) and (44) are exact but are not closed: the dynamics of low-order distributions are driven by those of higher-order distributions. Appropriate approximations are necessary to derive closed equations, such as molecular chaos, which will be studied in the next section.

\section{Derivation}

We here derive the lowest BBGKY hierarchal equation (40) for the reduced distribution function, starting from the financial Liouville equation (24). We first introduce the relative price from the c.m. as $r_{i} \equiv z_{i}-z_{\mathrm{c} . \mathrm{m} .}$. By making transformation $\Gamma=$ $\left(z_{1}, \ldots, z_{N} ; z_{\mathrm{c} . \mathrm{m} .}, p, \Delta p\right) \rightarrow \boldsymbol{\Gamma}_{r} \equiv\left(r_{1}, \ldots, r_{N} ; z_{\mathrm{c} . \mathrm{m} .}, p, \Delta p\right)$, the financial Liouville equation can be rewritten as

$$
\begin{aligned}
\frac{\partial P_{t}\left(\boldsymbol{\Gamma}_{r}\right)}{\partial t}= & {\left[-c \tanh \frac{\Delta p}{\Delta p^{*}} \partial_{\text {c.m. }}+\frac{\sigma^{2}}{2} \sum_{i=1}^{N}\left\{\partial_{i}+\frac{1}{N}\left(\partial_{\text {c.m. }}-\sum_{k=1}^{N} \partial_{k}\right)\right\}^{2}\right] P_{t}\left(\boldsymbol{\Gamma}_{r}\right)+\sum_{i, j} \frac{\sigma^{2}}{2}\left\{\delta\left(r_{i}-r_{j}\right) \delta\left(p-r_{i}-z_{\mathrm{c} . \mathrm{m} .}\right)\right.} \\
& \left.\times \int d \Delta p^{\prime}\left|\tilde{\partial}_{i j}\right| P_{t}\left(\boldsymbol{\Gamma}_{r}-\Delta \boldsymbol{\Gamma}_{i j ; r}^{\prime}\right)-\delta\left(r_{i}-r_{j}-\frac{L_{i}+L_{j}}{2}\right)\left|\tilde{\partial}_{i j}\right| P_{t}\left(\boldsymbol{\Gamma}_{r}\right)\right\}
\end{aligned}
$$

where we have used the chain rule for the variable transformation:

$$
\frac{\partial}{\partial z_{i}} \rightarrow \frac{\partial}{\partial r_{i}}, \quad \frac{\partial}{\partial z_{\mathrm{c} . \mathrm{m} .}} \rightarrow \frac{\partial}{\partial z_{\mathrm{c} . \mathrm{m} .}}-\sum_{k=1}^{N} \frac{\partial}{\partial r_{k}}
$$

We have also introduced $\Delta \Gamma_{i j ; r}^{\prime}=\Delta \Gamma_{i j ; r}^{\prime(0)}+\Delta \Gamma_{i j ; r}^{(1)}$ with

$$
\Delta \boldsymbol{\Gamma}_{i j ; r}^{(0)} \equiv\left(0, \ldots,-\frac{L_{i}}{2}, \ldots,+\frac{L_{j}}{2}, \ldots, 0 ; 0, \Delta p, \Delta p-\Delta p^{\prime}\right), \quad \Delta \boldsymbol{\Gamma}_{i j ; r}^{(1)} \equiv \frac{L_{i}-L_{j}}{2 N}(+1, \ldots,+1 ;-1,0,0) .
$$






(b)

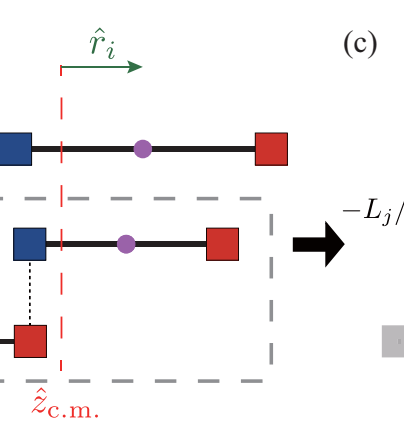

(c)



FIG. 8. Schematic of the three-body collision term $\mathcal{L}^{(i j k)}$. Let us assume that there is a collision between the traders $j$ and $k$. Because of the assumption of the binary interaction, the midprice $\hat{z}_{i}$ of the $i$ th trader does not move during this collision. On the other hand, the c.m. of this system $\hat{z}_{\text {c.m. }}$ moves through a short distance of $\Delta \hat{z}_{\text {c.m. }} \equiv \hat{z}_{\text {c.m. }}^{\text {pst }}-\hat{z}_{\text {c.m. }}=-\operatorname{sgn}\left(\hat{z}_{j}-\hat{z}_{k}\right)\left(L_{j}-L_{k}\right) / 2 N$ because of the requotation. The relative price $\hat{r}_{i}$ of the $i$ th trader indirectly moves through a short distance of $\Delta \hat{r}_{i} \equiv \hat{r}_{i}^{\mathrm{pst}}-\hat{r}_{i}=\Delta r_{i j ; s}^{(1)}$.

According to the definition of the one-body, two-body, and three-body reduced distributions (39), the lowest-order hierarchy is then derived as

$$
\begin{aligned}
\frac{\partial P_{t}^{i}\left(r_{i}\right)}{\partial t}= & \frac{\tilde{\sigma}^{2}}{2} \frac{\partial^{2} P_{t}^{i}\left(r_{i}\right)}{\partial r_{i}^{2}}+\sum_{s= \pm 1} \sum_{j \neq i} \frac{\sigma^{2}}{2}\left[\left.\left|\tilde{\partial}_{i j}\right| P_{t}^{i j}\left(r_{i}-\Delta r_{i j ; s}, r_{j}+\Delta r_{j i ; s}\right)\right|_{r_{i}=r_{j}}-\left.\left|\tilde{\partial}_{i j}\right| P_{t}^{i j}\left(r_{i}, r_{j}\right)\right|_{r_{i}-r_{j}=s\left(L_{i}+L_{j}\right) / 2}\right] \\
& +\left.\sum_{s= \pm 1} \sum_{j, k \neq i} \frac{\sigma^{2}}{2} \int d r_{j}\left[\left|\tilde{\partial}_{j k}\right| P_{t}^{i j k}\left(r_{i}-\Delta r_{j k ; s}^{(1)}, r_{j}, r_{k}\right)-\left|\tilde{\partial}_{j k}\right| P_{t}^{i j k}\left(r_{i}, r_{j}, r_{k}\right)\right]\right|_{r_{j}-r_{k}=s\left(L_{j}+L_{k}\right) / 2}
\end{aligned}
$$

with effective variance $\tilde{\sigma}^{2} \equiv \sigma^{2}(1-1 / N)$ and jump size $\quad \Delta r_{i j ; s} \equiv \Delta r_{i j ; s}^{(0)}+\Delta r_{i j ; s}^{(1)} \quad$ with $\quad \Delta r_{i j ; s}^{(0)} \equiv-s L_{i} / 2$, $\Delta r_{i j ; s}^{(1)} \equiv s\left(L_{i}-L_{j}\right) / 2 N$. Equation (40) is thus derived from Eq. (49) by introducing Liouville operators (41). Similarly, Eq. (44) can also be derived by integrating both sides of Eq. (24) with respect to $z_{1}, \ldots, z_{N}$.

\section{Remark on the three-body collision term}

We remark on the emergence of the three-body collision term $\mathcal{L}^{(i j k)}$ in the BBGKY hierarchy (40), which is slightly different from the conventional BBGKY hierarchy (4a). This term appears because our kinetic theory is formulated on the basis of the relative price $\hat{r}_{i}$. To understand this point, let us consider the movement of the relative price $\hat{r}_{i}$ of the $i$ th trader during collision between traders $j$ and $k$ (see Fig. 8 for a schematic of three-body collision). While the midprice $\hat{z}_{i}$ of the $i$ th trader does not move during the collision between

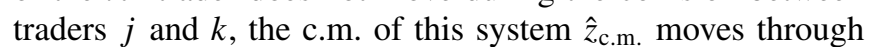
a distance of $\Delta \hat{z}_{\mathrm{c} . \mathrm{m} .} \equiv \hat{z}_{\mathrm{c} . \mathrm{m} .}^{\mathrm{pst}}-\hat{z}_{\mathrm{c} . \mathrm{m} .}=-\operatorname{sgn}\left(\hat{z}_{j}-\hat{z}_{k}\right)\left(L_{j}-\right.$ $\left.L_{k}\right) / 2 N$. The relative price $\hat{r}_{i}$ thus moves indirectly through a distance of $\Delta \hat{r}_{i} \equiv \hat{r}_{i}^{\mathrm{pst}}-\hat{r}_{i}=-\Delta \hat{z}_{\mathrm{c} . \mathrm{m} .}=\Delta r_{j k ; s}^{(1)}$, which appears in the three-body collision operator (41c). This effect is intuitively small for the large- $N$ limit and is finally shown to be irrelevant to leading-order (LO) and next-leading-order (NLO) approximations as discussed later.

\section{MAIN RESULT 2: MESOSCOPIC DESCRIPTION}

From microscopic dynamics, we have derived the BBGKY hierarchal equation (40) for the mesoscopic description of the
HFT model in a parallel manner to the conventional BBGKY hierarchal equations (4). Here we proceed to derive the closed mean-field model for the mesoscopic description, which will be finally shown useful in systematic understanding of the order-book profile.

\section{A. Financial Boltzmann equation}

We here derive a closed equation for the one-body distribution function by assuming a mean-field approximation. The one-body and two-body distribution functions $\phi_{t}^{L}(r)$ and $\phi_{t}^{L L^{\prime}}\left(r, r^{\prime}\right)$ are introduced conditional on the traders' spreads $L$ and $L^{\prime}$, such that $\phi_{t}^{L_{i}}(r)=P_{t}^{i}(r)$ and $\phi_{t}^{L_{i} L_{j}}\left(r, r^{\prime}\right)=$ $P_{t}^{i j}\left(r, r^{\prime}\right)$. Let us approximately truncate the two-body correlation as

$$
\phi_{t}^{L L^{\prime}}\left(r, r^{\prime}\right) \approx \phi_{t}^{L}(r) \phi_{t}^{L^{\prime}}\left(r^{\prime}\right),
$$

which corresponds to molecular chaos (5), the standard approximation in kinetic theory. The validity of this approximation will be numerically evaluated in Sec. V B. A closed mean-field equation for the one-body distribution $\phi_{t}^{L}(r)$ is thus obtained as

$$
\begin{aligned}
\frac{\partial \phi_{t}^{L}}{\partial t} \approx & \frac{\sigma^{2}}{2} \frac{\partial^{2} \phi_{t}^{L}}{\partial r^{2}}+N \sum_{s= \pm 1} \int_{L_{\min }}^{L_{\max }} d L^{\prime} \rho_{L^{\prime}} \\
& \times\left[J_{t ; s}^{L L^{\prime}}(r+s L / 2)-J_{t ; s}^{L L^{\prime}}(r)\right], \\
J_{t ; s}^{L L^{\prime}}(r)= & \left.\frac{\sigma^{2}}{2}\left|\tilde{\partial}_{r r^{\prime}}\right| \phi_{t}^{L}(r) \phi_{t}^{L^{\prime}}\left(r^{\prime}\right)\right|_{r-r^{\prime}=s\left(L+L^{\prime}\right) / 2}
\end{aligned}
$$

with mean-field probability flux $J_{t ; s}^{L L^{\prime}}(r)$ for $s= \pm 1$. The systematic derivation of this equation is the third main result 
in this paper (see the derivation below). Equation (51) is a closed equation for the one-body distribution function, and corresponds to the Boltzmann equation in molecular kinetic theory [see Fig. 1(b)]. Equation (51) is therefore called the financial Boltzmann equation in this paper. Here the dummy variable $s=+1(s=-1)$ implies the transactions as a bidder (an asker), and the integrals on the right-hand side (rhs) correspond to the collision integrals in the standard Boltzmann equation (6). Remarkably, Eq. (51) is derived from a systematic calculation from the Liouville equation (24), whereas it was originally introduced with a rather heuristic discussion in our previous paper [46].

\section{Derivation}

Here we derive the financial Boltzmann equation (51) from the financial BBGKY hierarchy (40). To simplify the hierarchal equation (40), we use the symmetry among the traders in terms of the spread: when the spreads are equal for $i$ th and $j$ th traders, their one-body distributions are also equal, namely,

$$
L_{i}=L_{j} \Longrightarrow P_{t}^{i}(r)=P_{t}^{j}(r)
$$

Furthermore, there are also symmetries for the two-body and three-body distributions such that

$$
L_{i}=L_{k}, \quad L_{j}=L_{l} \Longrightarrow P_{t}^{i j}\left(r, r^{\prime}, r^{\prime \prime}\right)=P_{t}^{k l}\left(r, r^{\prime}, r^{\prime \prime}\right)
$$

and

$$
\begin{aligned}
& L_{i}=L_{l}, \quad L_{j}=L_{m}, \\
& L_{k}=L_{n} \Longrightarrow P_{t}^{i j k}\left(r, r^{\prime}, r^{\prime \prime}\right)=P_{t}^{l m n}\left(r, r^{\prime}, r^{\prime \prime}\right) .
\end{aligned}
$$

On the basis of these symmetries, we introduce the conditional distributions on spreads. We assume that the number of traders is large enough such that we can approximately regard the spreads as continuously distributed. In other words, the spread distribution $\rho_{L} \equiv \sum_{i=1}^{N} \delta\left(L-L_{i}\right) / N$ is an approximately continuous function. We also assume that $\rho_{L}=0$ for $L \notin\left[L_{\min }, L_{\max }\right]$. The one-body and two-body distributions $\phi_{t}^{L}(r)$ and $\phi_{t}^{L L^{\prime}}\left(r, r^{\prime}\right)$ are defined conditional on spreads $L$ and $L^{\prime}$ by

$$
\phi_{t}^{L_{i}}(r) \equiv P_{t}^{i}(r), \quad \phi_{t}^{L_{i} L_{j}}\left(r, r^{\prime}\right) \equiv P_{t}^{i j}\left(r, r^{\prime}\right) .
$$

Here, we make the following approximations for $N \rightarrow \infty$ :

(1) The effective variance $\tilde{\sigma}^{2}=\sigma^{2}(1-1 / N)$ is approximated as

$$
\tilde{\sigma}^{2} \approx \sigma^{2}
$$

(2) The discrete sums are approximated as continuous integrals:

$$
\begin{aligned}
& \sum_{j}(\ldots) \approx N \int_{L_{\min }}^{L_{\max }} d L^{\prime} \rho_{L^{\prime}}(\ldots), \\
& \sum_{j, k}(\ldots) \approx N^{2} \int_{L_{\min }}^{L_{\max }} d L^{\prime} d L^{\prime \prime} \rho\left(L^{\prime}\right) \rho\left(L^{\prime \prime}\right)(\ldots) .
\end{aligned}
$$

(3) The relatively small displacement $\Delta r_{i j ; s}^{(1)}$ is negligible:

$$
\left|\Delta r_{i j ; s}^{(1)}\right| \ll\left|\Delta r_{i j ; s}^{(0)}\right| .
$$

On the basis of these approximations, the lowest hierarchal equation (40) can be rewritten as

$$
\begin{aligned}
\frac{\partial \phi_{t}^{L}(r)}{\partial t} \approx & \frac{\sigma^{2}}{2} \frac{\partial^{2} \phi_{t}^{L}(r)}{\partial r^{2}}+N \sum_{s= \pm 1} \int d L^{\prime} \rho_{L^{\prime}} \frac{\sigma^{2}}{2} \\
& \times\left[\left.\left|\tilde{\partial}_{r r^{\prime}}\right| \phi_{t}^{L L^{\prime}}\left(r-\frac{s L}{2}, r^{\prime}+\frac{s L^{\prime}}{2}\right)\right|_{r=r^{\prime}}\right. \\
& \left.-\left.\left|\tilde{\partial}_{r r^{\prime}}\right| \phi_{t}^{L L^{\prime}}\left(r, r^{\prime}\right)\right|_{r-r^{\prime}=s\left(L+L^{\prime}\right) / 2}\right],
\end{aligned}
$$

which was heuristically derived in our previous paper [46]. Here we have ignored the correction terms of $O\left(N^{-1}\right)$. The financial Boltzmann equation (51) is then derived by making the mean-field approximation (50) to Eq. (59). Note that the three-body correlation term in Eq. (40) is finally irrelevant under these assumptions. The consistency of the assumption (58) is examined using the NLO solution (61) of Eq. (51) in Appendix B.

\section{B. Solution}

Let us focus on the steady solution $\phi^{L}(r) \equiv \lim _{t \rightarrow \infty} \phi_{t}^{L}(r)$ to the financial Boltzmann Eq. (51). Equation (51) can be asymptotically solved for the liquid market $N \gg 1$ using the boundary-layer analysis. To understand our picture, let us introduce one-body PDFs $f_{B}^{i}(r)$ and $f_{A}^{i}(r)$ for the relative bid and ask prices from the c.m. for the trader $i$ (see Fig. 9(a) for the schematic profiles): ${ }^{6}$

$$
\begin{aligned}
& f_{B}^{i}(r) \equiv\left\langle\delta\left(\hat{b}_{i}-\hat{z}_{\mathrm{c} . \mathrm{m} .}-r\right)\right\rangle=\phi^{L_{i}}\left(r+L_{i} / 2\right), \\
& f_{A}^{i}(r) \equiv\left\langle\delta\left(\hat{a}_{i}-\hat{z}_{\mathrm{c} . \mathrm{m} .}-r\right)\right\rangle=\phi^{L_{i}}\left(r-L_{i} / 2\right) .
\end{aligned}
$$

The basic idea is that most of the transactions will occur near the c.m. for $N \gg 1$; the c.m. will be a strict transaction barrier at which any bid and ask order will be transacted instantaneously for infinite $N$ (i.e., the LO approximation), whereas the c.m. will be a stochastic transaction barrier near which a bid and ask order will be transacted with high probability for finite $N$ (i.e., the NLO approximation). In Fig. 9(a), we illustrate that a bid (ask) order can go beyond the c.m. with low probability for finite $N$, which is reflected as the tail near $r=0$. In physical mathematics, such finite but small probability leakage can be captured by boundary-layer analysis, which was historically developed for fluid dynamics and was generalized for a singular perturbation theory. In the following, we found an asymptotic solution in terms of $1 / N$ within the spirit of boundary-layer analysis.

This picture can be rephrased for the one-body $\operatorname{PDF} \phi^{L}(r)$ for the relative midprice as illustrated in Fig. 9(b). The transaction barrier at the c.m. in Fig. 9(a) corresponds to the barriers at $r= \pm L / 2$ with the boundary layer of thickness $\varepsilon$ in Fig. 9(b), characterizing the small probabilistic leak near

\footnotetext{
${ }^{6}$ Since Fig. 9(a) is a schematic of the average bid and ask orderbook profiles measured from the c.m., their slight overlap does not indicate immediate transactions. We note that there is no overlap in principle between the simultaneous snapshot of the bid and ask order books for this model.
} 
(a)



(b)



FIG. 9. Schematic of the NLO boundary layer solution (61). (a) Schematic of the bid and ask average order-book profiles $f_{B}^{i}(r)$ and $f_{A}^{i}(r)$ for the $i$ th trader. Most of transactions will occur at the c.m. $r=0$ for infinite $N$ since the market is sufficiently liquid. On the other hand, for a finite large $N \gg 1$, a small portion of bid and ask orders can go beyond the c.m. with low probability, which is reflected as the tail near $r \simeq 0$ with thickness of the boundary layer $\varepsilon=L_{\rho}^{*} / 2 \sqrt{N}$. (b) Schematic of the midprice average order-book profile $\phi^{L}(r)$. The tail near $r \simeq \pm L / 2$ rapidly decays with the thickness of the boundary layer $\varepsilon$. The peak is also rounded with the thickness of $\varepsilon$ because requoted orders will be submitted near the peak, after transactions near the tail.

the tails. ${ }^{7}$ We found the following global asymptotic steady solution satisfying Eq. (51) up to the NLO approximation:

$$
\phi^{L}(r) \simeq \frac{4 \varepsilon}{L^{2}}\left[\mathcal{F}\left(\frac{|r|-L / 2}{\varepsilon}\right)-2 \mathcal{F}\left(\frac{|r|}{\varepsilon}\right)\right],
$$

which we call the NLO boundary-layer solution. Here we introduced

$$
\begin{aligned}
\frac{1}{L_{\rho}^{* 2}} & \equiv \int_{L_{\min }}^{L_{\max }} \frac{d L \rho_{L}}{L^{2}}, \quad \varepsilon \equiv \frac{L_{\rho}^{*}}{2 \sqrt{N}}, \\
\mathcal{F}(r) & \equiv \frac{e^{-r^{2} / 2}}{\sqrt{2 \pi}}-\frac{r}{2} \operatorname{erfc}\left(\frac{r}{\sqrt{2}}\right)
\end{aligned}
$$

with complementary error function $\operatorname{erfc}(r) \equiv$ $(2 / \sqrt{\pi}) \int_{r}^{\infty} d t e^{-t^{2}}$. The validity of this solution can be checked by direct substitution into Eq. (51) (see Appendix A for the calculation). The LO solution is given by the tent function:

$$
\psi^{L}(r) \equiv \lim _{N \rightarrow 0} \phi^{L}(r)=\frac{4}{L^{2}} \max \left\{\frac{L}{2}-|r|, 0\right\},
$$

which is a reasonable solution since the tent function is the steady solution for the Brownian motion surrounded by strict hopping barriers (see the Supplemental Material of Ref. [46]). Although the LO solution (63) is sufficient to understand the average order-book profile as discussed in Sec. V C, the details of the NLO solution (61) are necessary to understand the dynamics of the financial Langevin equation as shown in Sec. VI.

\section{Technical issues}

Here we explain the boundary condition for our asymptotic analysis. We introduce cutoffs for the boundaries at $r=$ $\pm L_{\text {cut }} / 2$ and make the following four assumptions:

(1) Equation (51) is valid only for $r \in\left[-L_{\text {cut }} / 2, L_{\text {cut }} / 2\right]$.

(2) The cutoff is taken sufficiently large: $L_{\text {cut }}>L_{\max }$.

\footnotetext{
${ }^{7}$ The peak regime is also under the influence of the boundary layer since the trader's midprice will return near the peak after price matching.
}

(3) The probability is zero beyond the boundary: $\phi_{t}^{L}(r)=$ 0 for $r \notin\left[-L_{\text {cut }} / 2, L_{\text {cut }} / 2\right]$.

(4) The boundaries are the reflecting barriers, which ensure the conservation of the probability:

$$
\left.\frac{\partial \phi_{t}^{L}(r, t)}{\partial r}\right|_{r= \pm L_{\mathrm{cut}} / 2}=0 \Longrightarrow \frac{\partial}{\partial t} \int_{-L_{\mathrm{cut}} / 2}^{L_{\mathrm{cut}} / 2} d r \phi_{t}^{L}(r, t)=0 .
$$

The probability conservation (64) can be proved under this boundary condition as shown in Appendix $C$. The cutoff parameter is finally taken at infinity as $L_{\text {cut }} \rightarrow \infty$, and the main results in this paper do not depend on $L_{\text {cut }}$.

We also note another related technical issue for the meanfield solution (63). The large-number limit $N \rightarrow \infty$ is taken first; the $L_{\max } \rightarrow \infty$ limit is taken after the $L_{\text {cut }} \rightarrow \infty$ limit to conserve the second assumption $L_{\text {cut }}>L_{\max }$. Therefore, the steady solution (63) technically implies

$$
\psi^{L}(r) \equiv \lim _{t \rightarrow \infty} \lim _{L_{\max } \rightarrow \infty} \lim _{L_{\text {cut }} \rightarrow \infty} \lim _{N \rightarrow \infty} \phi_{t}^{L}(r) .
$$

\section{Average order-book profile}

Considering that the ask order-book snapshot is defined by $\hat{f}_{A}(r) \equiv \sum_{i=1}^{N} \delta\left(\hat{a}_{i}-\hat{z}_{\mathrm{c} . \mathrm{m} .}-r\right)$, the average ask orderbook profile $f_{A}(r) \equiv\left\langle\hat{f}_{A}(r)\right\rangle=\sum_{i=1}^{N} f_{A}^{i}(r)$ is given by the superposition of the tent function to leading order:

$$
f_{A}(r) \simeq \int_{L_{\min }}^{L_{\max }} d L \rho_{L} \psi^{L}(r-L / 2)
$$

(see Fig. 10). The average order-book profile has a symmetry, such that $f_{B}(r)=f_{A}(-r)$ for the average bid order-book $f_{B}(r)$.

We numerically examined the theoretical order-book profile (66) for two examples, the $\delta$-distributed spread

$$
\rho_{L}=\delta\left(L-L^{*}\right)
$$

and the $\gamma$-distributed spread

$$
\rho_{L}=\frac{L^{3} e^{-L / L^{*}}}{6 L^{* 4}}
$$

with the characteristic length parameter $L^{*}$. The numerical implementation of the trend-following HFT model is as 


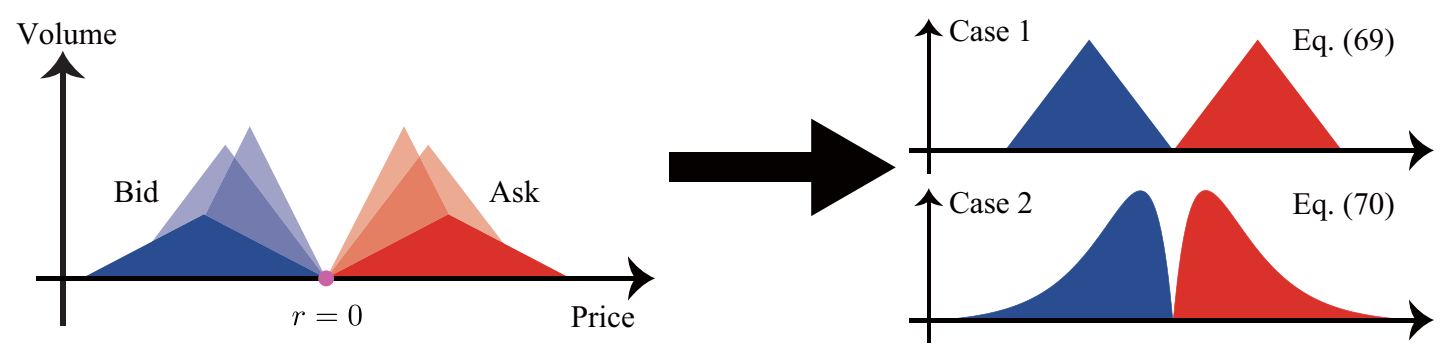

FIG. 10. Average order-book profile is given by the superposition (66) of the tent function (63). For the $\delta$-distributed spread (Case 1), the profile is the tent function (69). For the $\gamma$-distributed spread (Case 2), the profile obeys Eq. (70).

follows: The length and time units of this system are given by $L^{*}$ and $L^{* 2} /\left(\sigma^{2} N\right)$, respectively. We performed the Monte Carlo simulation for various numbers of traders $N$ under a fixed discretization time step $\Delta t=0.01 L^{* 2} /\left(\sigma^{2} N\right)$. For initialization, we first ran the simulation for the time interval of $10 L^{* 2} / \sigma^{2}$ and then ran the simulation again to take samples. The simulation time was set to be $10^{5}$ ticks.

\section{Case 1: $\delta$-distributed spread}

Let us first consider the case of the $\delta$-distributed spread (67). The corresponding average order-book profile is given by the tent function

$$
f_{A}(r)=\psi^{L^{*}}\left(r-L^{*} / 2\right)=\frac{4}{L^{2 *}} \max \left\{\frac{L^{*}}{2}-\left|r-\frac{L^{*}}{2}\right|, 0\right\} .
$$

We have numerically examined the validity of this formula in Fig. 11(a), which shows numerical agreement with our formula (69). The LO solution (69) works quite well for the description of the order-book profile, and the numerical convergence in Fig. 11(a) implies that Eq. (69) might be exactly valid for $N \rightarrow \infty$.

\section{Case 2: $\gamma$-distributed spread}

The formula (66) works well even for $L_{\text {min }} \rightarrow 0$ and $L_{\max } \rightarrow \infty$ when the integrals converge. For the $\gamma$-distributed spread (68), we obtain

$$
\begin{aligned}
f_{A}(r) & =\frac{1}{L^{*}} \psi\left(\frac{r}{L^{*}}\right), \\
\psi(r) & \equiv \frac{4}{3} e^{-\frac{3 r}{2}}\left[(2+r) \sinh \frac{r}{2}-\frac{r}{2} e^{-\frac{r}{2}}\right],
\end{aligned}
$$

which was empirically validated through single-trajectory analysis of individual traders in our previous work [46]. We have numerically examined the validity of this formula in Fig. 11(b), showing numerical agreement with our formula (70). The numerical convergence in Fig. 11(b) implies that the LO solution (70) might be also exact for $N \rightarrow \infty$.

\section{MAIN RESULT 3: MACROSCOPIC DESCRIPTION}

In this section, we derive the stochastic equations for the macroscopic dynamics of this system from the BBGKY hierarchal equation (44) in the parallel method to the master Boltzmann equation (8) for physical Brownian motion.

\section{A. Master Boltzmann equation for financial Brownian motion}

On the basis of the financial BBGKY hierarchy (44) for the macroscopic dynamics, we derive a closed dynamical equation for the macroscopic variables $\boldsymbol{Z} \equiv\left(z_{\mathrm{c} . \mathrm{m} .}, p, \Delta p\right)$. Here, we first make the assumption of molecular chaos,

$$
P_{t}^{i j}\left(z_{i}, z_{j} ; \boldsymbol{Z}\right) \approx \phi_{t}^{L_{i}}\left(z_{i}-z_{\mathrm{c} . \mathrm{m} .}\right) \phi_{t}^{L_{j}}\left(z_{j}-z_{\mathrm{c} . \mathrm{m} .}\right) P_{t}(\boldsymbol{Z}) .
$$

Using the NLO solution (61), we deduce a closed master Boltzmann equation for the macroscopic dynamics (see the detailed calculation below):

$$
\frac{\partial P_{t}(\boldsymbol{Z})}{\partial t} \approx\left(\mathcal{L}_{\mathrm{c} . \mathrm{m} .}^{\mathrm{a}}+\mathcal{L}_{\mathrm{c} . \mathrm{m} .}^{\mathrm{c} ; \mathrm{MF}}\right) P_{t}(\boldsymbol{Z})
$$

where the mean-field collision Liouville operator for the macroscopic variables $\mathcal{L}_{\mathrm{c} . \mathrm{m} \text {. }}^{\mathrm{c} ; \mathrm{MF}}$ is defined by

$$
\mathcal{L}_{\mathrm{c} . \mathrm{m} .}^{\mathrm{c} \text { MF }} P_{t} \equiv \int d \boldsymbol{X}\left\{W(\boldsymbol{Z} \mid \boldsymbol{X}) P_{t}(\boldsymbol{X})-W(\boldsymbol{X} \mid \boldsymbol{Z}) P_{t}(\boldsymbol{Z})\right\}
$$

with transition rate $W(\boldsymbol{Z} \mid \boldsymbol{X})$ conditional on the state variables before the jump $\boldsymbol{X} \equiv\left(z_{\mathrm{c} . \mathrm{m} .}^{\prime}, p^{\prime}, \Delta p^{\prime}\right)$ specified by

$$
\begin{aligned}
W(\boldsymbol{Z} \mid \boldsymbol{X}) \equiv & \frac{1}{\tau^{*}} \mathcal{N}\left(p-z_{\mathrm{c} . \mathrm{m} .} ; \frac{L_{\rho}^{* 2}}{4 N}\right) \\
& \times w_{N}\left(z_{\mathrm{c} . \mathrm{m} .}-z_{\mathrm{c} . \mathrm{m} .}^{\prime}\right) \delta\left(\Delta p-p+p^{\prime}\right)
\end{aligned}
$$

and volume element $d \boldsymbol{X} \equiv d z_{\text {c.m. }}^{\prime} d p^{\prime} d \Delta p^{\prime}$. Here, $\mathcal{N}\left(x ; \sigma^{2}\right)$ is the Gaussian distribution, $w_{N}(y)$ is jump size distribution, and $\tau^{*}$ is the mean transaction interval defined by

$$
\begin{aligned}
\mathcal{N}\left(x ; \sigma^{2}\right) & \equiv \frac{e^{-x^{2} / 2 \sigma^{2}}}{\sqrt{2 \pi \sigma^{2}}}, \\
w_{N}(y) & \equiv \int_{-\infty}^{\infty} \frac{2 N L_{\rho}^{* 4} d L}{L^{2}(L+2 N y)^{2}} \rho_{L} \rho_{(L+2 N y)}, \\
\tau^{*} & \equiv \frac{1}{\int d \boldsymbol{Z} W(\boldsymbol{Z} \mid \boldsymbol{X})} \simeq \frac{L_{\rho}^{* 2}}{2 N \sigma^{2}} .
\end{aligned}
$$

We note that $w_{N}(y)$ satisfies the scaling of the system size expansion [16]:

$$
\tilde{w}(y) \equiv \frac{1}{N} w_{N}\left(\frac{y}{N}\right)
$$

is an $N$-independent non-negative function. This character is useful for an asymptotic expansion in terms of $1 / N$ as will be shown later.

Because any master equation corresponds to a set of SDEs [60], the dynamics of the master Boltzmann equation (79) can 
(a) $f_{A}\left(r / L^{*}\right) / L^{*}$



(b) $f_{A}\left(r / L^{*}\right) / L^{*}$

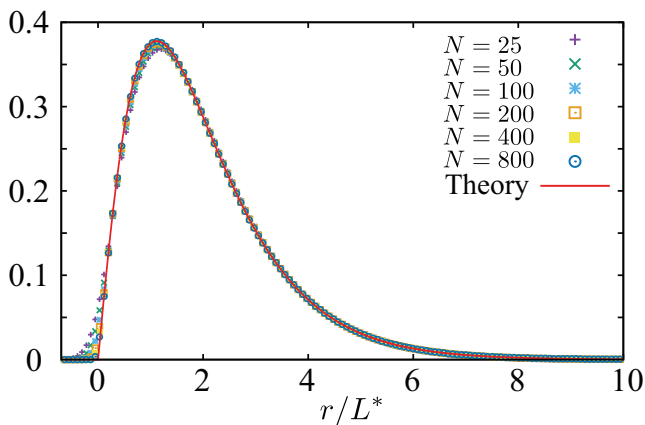

FIG. 11. Numerical validation of our formula (66) for the average order-book profile for two examples. (a) Numerical average ask orderbook profile for the $\delta$-distributed spread $\rho_{L}=\delta\left(L-L^{*}\right)$, showing the agreement with the theoretical formula (69) for $N \rightarrow \infty$. (b) Numerical average ask order-book profile for the $\gamma$-distributed spread $\rho_{L}=L^{3} e^{-L / L^{*}} / 6 L^{* 4}$, showing the agreement with the theoretical formula (70) for $N \rightarrow \infty$.

be mapped to those of a set of SDEs

$$
\begin{aligned}
\frac{d \hat{z}_{\text {c.m. }}}{d t} & =c \tanh \frac{\Delta \hat{p}}{\Delta p^{*}}+\frac{\sigma}{\sqrt{N}} \hat{\xi}^{\mathrm{G}}+\left(\hat{z}_{\mathrm{c} . \mathrm{m} .}^{\mathrm{pst}}-\hat{z}_{\mathrm{c.m} .}\right) \hat{\xi}_{\tau^{*}}^{\mathrm{P}}, \\
\frac{d \hat{p}}{d t} & =\left(\hat{p}^{\mathrm{pst}}-\hat{p}\right) \hat{\xi}_{\tau^{*}}^{\mathrm{P}}, \quad \frac{d \Delta \hat{p}}{d t}=\left(\Delta \hat{p}^{\mathrm{pst}}-\Delta \hat{p}\right) \hat{\xi}_{\tau^{*}}^{\mathrm{P}}
\end{aligned}
$$

with white Gaussian noise $\hat{\xi}^{\mathrm{G}}$ of unit variance and white Poisson noise $\hat{\xi}_{\tau^{*}}^{\mathrm{P}}$ of mean interval $\tau^{*}$. The postcollisional states are given by $\left(\hat{z}_{\mathrm{c} . \mathrm{m}}^{\mathrm{pst}}, \hat{p}^{\mathrm{pst}}, \Delta \hat{p}^{\mathrm{pst}}\right) \equiv$ $\left(\hat{z}_{\mathrm{c} . \mathrm{m} .}+\hat{v}[T] / N, \hat{z}_{\mathrm{c} . \mathrm{m} .}^{\mathrm{pst}}+\left(L_{\rho}^{*} / 2 \sqrt{N}\right) \hat{\xi}[T], \hat{p}^{\mathrm{pst}}-\hat{p}\right)$ at the tick time $T$. Here, $\hat{v}[T]$ is a discrete-time white noise term whose PDF is given by $\tilde{w}(v)$ and $\hat{\xi}[T]$ is a discrete-time white Gaussian noise with unit variance.

\section{Derivation}

The master Boltzmann equation (72) is derived from the BBGKY hierarchal equation (44) for macroscopic variables. By applying a mean-field approximation (71) to Eq. (44), we obtain

$$
\begin{aligned}
\frac{\partial P_{t}\left(z_{\text {c.m. } .}, p, \Delta p\right)}{\partial t}= & \left.-c \tanh \frac{\Delta p}{\Delta p^{*}} \partial_{\text {c.m. }}+\frac{\sigma^{2}}{2 N} \partial_{\text {c.m. }}^{2}\right] P_{t}\left(z_{\text {c.m. } .}, p, \Delta p\right)+\frac{N^{2} \sigma^{2}}{2} \int d L d L^{\prime} \rho_{L} \rho_{L^{\prime}} \\
& \times\left\{\left.\int d \Delta p^{\prime}\left|\tilde{\partial}_{r r^{\prime}}\right| \phi^{L}\left(r+\frac{L}{2}-z_{\text {c.m. }}\right) \phi^{L^{\prime}}\left(r^{\prime}-\frac{L^{\prime}}{2}-z_{\text {c.m. }}\right)\right|_{r=r^{\prime}=p} P_{t}\left(z_{\text {c.m. }}+\frac{L-L^{\prime}}{2 N}, p-\Delta p, \Delta p^{\prime}\right)\right. \\
& \left.-\left.P\left(z_{\text {c.m. }}, p, \Delta p\right) \int d r\left|\tilde{\partial}_{r r^{\prime}}\right| \phi^{L}(r) \phi^{L^{\prime}}\left(r^{\prime}\right)\right|_{r=r^{\prime}+\left(L+L^{\prime}\right) / 2}\right\} .
\end{aligned}
$$

Using the NLO solution (61), we deduce

$$
\frac{\partial P_{t}(\boldsymbol{Z})}{\partial t}=\mathcal{L}_{\mathrm{c} . \mathrm{m} .}^{\mathrm{a}} P_{t}(\boldsymbol{Z})+\frac{1}{\tau^{*}}\left[\mathcal{N}\left(p-z_{\mathrm{c} . \mathrm{m} .} ; \frac{L_{\rho}^{* 2}}{4 N}\right) \int d \Delta p^{\prime} d y w_{N}(y) P_{t}\left(z_{\mathrm{c} . \mathrm{m} .}-y, p-\Delta p, \Delta p^{\prime}\right)-P_{t}(\boldsymbol{Z})\right] .
$$

By introducing $\quad \boldsymbol{X} \equiv\left(z_{\mathrm{c} . \mathrm{m} .}^{\prime}, p^{\prime}, \Delta p^{\prime}\right) \equiv\left(z_{\mathrm{c} . \mathrm{m} .}-y, p-\right.$ $\left.\Delta p, \Delta p^{\prime}\right)$ and the transition rate $W(\boldsymbol{Z} \mid \boldsymbol{X})$, this equation can be rewritten into Eq. (72), which is a standard differential form of the Chapman-Kolmogorov equation [13].

\section{B. Financial Langevin equation}

We have derived the stochastic dynamics for the three macroscopic variables $\hat{Z}=\left(\hat{z}_{\text {c.m. }}, \hat{p}, \Delta \hat{p}\right)$ as the master equation (72) [or equivalently SDEs (77)] in the continuous time $t$. We next simplify the dynamics (72) of the three macroscopic variables into that of a single macroscopic variable $\Delta \hat{p}$ in the tick time $T$. In the tick time $T$, the dynamical equation for the price movement $\Delta \hat{p}$ is given by

$$
\Delta \hat{p}[T+1]=\underbrace{c \hat{\tau}[T] \tanh \frac{\Delta \hat{p}[T]}{\Delta p^{*}}}_{\text {Trend following }}+\underbrace{\Delta \hat{\xi}[T]}_{\text {Zigzag }}+\underbrace{\hat{\zeta}[T]}_{\text {Random }},
$$

where $\hat{\tau}[T] \equiv \hat{t}[T+1]-\hat{t}[T]$ is time interval between transaction, $\Delta \hat{\xi}[T]$ is the zigzag noise of order $N^{-1 / 2}$, and $\hat{\zeta}[T]$ is a random noise of order $N^{-1}$ (see the derivation below). The systematic derivation of Eq. (80) is the fourth main result of this paper. Equation (80) corresponds to the conventional Langevin equation (9), and is thus called the financial Langevin equation in this paper.

Within the mean-field approximation, we can specify all the statistics of the random noise terms from analytics. The time interval $\hat{\tau}[T]$ is given by the exponential random number 
with mean interval $\tau^{*}$,

$$
P(\tau)=\frac{1}{\tau^{*}} e^{-\tau / \tau^{*}}, \quad \tau^{*}=\frac{L_{\rho}^{* 2}}{2 N \sigma^{2}} .
$$

The zigzag noise $\Delta \hat{\xi}[T]$ is defined by the difference of two Gaussian random numbers as

$$
\Delta \hat{\xi}[T] \approx \frac{L_{\rho}^{*}}{2 \sqrt{N}}(\hat{\xi}[T]-\hat{\xi}[T-1])=O\left(N^{-1 / 2}\right),
$$

where $\hat{\xi}[T]$ is a discrete-time white Gaussian noise with unit variance. The random noise term $\hat{\zeta}[T]$ is specified as

$$
\hat{\zeta}[T] \approx \sigma \sqrt{\frac{\hat{\tau}(T)}{N}} \hat{\mu}[T]+\frac{1}{N} \hat{v}[T]=O\left(N^{-1}\right),
$$

where $\hat{\mu}[T]$ is a discrete-time white Gaussian noise with unit variance and $\hat{v}[T]$ is a discrete-time white noise term whose PDF is given by $\tilde{w}(v)$.

Here we describe the concrete statistics of the noise in Eq. (80) for two specific cases as follows:

Case 1: $\delta$-distributed spread. For the $\delta$-distributed spread (67), we obtain

$$
\tilde{w}(y)=\delta(y), \quad \tau^{*}=\frac{L^{* 2}}{2 N \sigma^{2}}, \quad L_{\rho}^{* 2}=L^{* 2},
$$

which implies the absence of $\hat{v}(T)$ for the $\delta$-distributed spread [i.e., $\hat{v}(T)=0$ ]. This is natural because the c.m. is conserved during transaction for this special case.

Case 2: $\gamma$-distributed spread. For the $\gamma$-distributed spread (68), we obtain

$$
\tilde{w}(y)=\frac{L^{*}+2|y|}{2 L^{* 2}} e^{-2|y| / L^{*}}, \quad \tau^{*}=\frac{3 L^{* 2}}{N \sigma^{2}}, \quad L_{\rho}^{* 2}=6 L^{* 2} .
$$

We next discuss the interpretation of each term on the rhs of Eq. (80). The trend-following term induces the collective motion of the order book and thus keeps the price movement in the same direction for a certain time interval similarly to inertia in physics. On the other hand, the zigzag noise term exhibits one-tick negative autocorrelation, such that

$$
C_{\Delta \hat{\xi}}[K] \equiv \frac{\langle\Delta \hat{\xi}[T+K] \Delta \hat{\xi}[T]\rangle}{\left\langle\Delta \hat{\xi}[T]^{2}\right\rangle} \approx \begin{cases}1 & (K=0), \\ -1 / 2 & (K=1) \\ 0 & (K \geqslant 2)\end{cases}
$$

and has the effect to change the price movement direction alternately. In this sense, the trend-following term and the zigzag noise have the opposite effect to each other; the balance between their strengths is crucial for the qualitative behavior of the market price dynamics. The random noise term $\hat{\zeta}[T]$ originates from the slow dynamics of the c.m.: $\sigma(\tau[T] / N)^{1 / 2} \hat{\mu}[T]$ is the diffusion term of the c.m. during a transaction time interval $\hat{\tau}[T]$ and $\hat{v}[T] / N$ is the movement term of the c.m. by requotation jumps of traders' midprices after transaction.

\section{Derivation}

Here we simplify the three SDEs (77) for $\left(\hat{z}_{\text {c.m. }}, \hat{p}, \Delta \hat{p}\right)$ in continuous time $t$ into a single SDE (80) for price movement $\Delta \hat{p}$ in discrete time. According to the set of SDEs (77), $\hat{z}_{\mathrm{c} . \mathrm{m}}$. exhibits random walks with a constant drift in the absence of transactions, without updates for $\hat{p}$ and $\Delta \hat{p}$. The movement of the c.m. between transactions (i.e., the time interval $[\hat{t}[T]+$ $\epsilon, \hat{t}[T+1]-\epsilon]$ with the infinitesimal positive number $\epsilon$ ) is then basically determined by the time interval $\hat{\tau}[T] \equiv \hat{t}[T+$ $1]-\hat{t}[T]$ as

$$
\begin{aligned}
& \hat{z}_{\text {c.m. }}(\hat{t}[T+1]-\epsilon)-\hat{z}_{\text {c.m. }}(\hat{t}[T]+\epsilon) \\
& \quad=c \hat{\tau}[T] \tanh \frac{\Delta \hat{p}}{\Delta p^{*}}+\sqrt{\frac{\sigma^{2} \hat{\tau}[T]}{N}} \hat{\mu}[T]+O(\epsilon)
\end{aligned}
$$

with Gaussian random noise $\hat{\mu}[T]$ with unit variance. Within the mean-field approximation, $\hat{\tau}[T]$ is an exponential random number with mean interval $\tau^{*}$. At the instance of the transaction at time $\hat{t}[T+1]$, there is a jump originating from the Poisson noise term $\left(\hat{z}_{\text {c.m. }}^{\mathrm{pst}}-\hat{z}_{\text {c.m. }}\right) \hat{\xi}_{\tau^{*}}^{\mathrm{P}}$,

$\hat{z}_{\mathrm{c} . \mathrm{m} .}(\hat{t}[T+1]+\epsilon)-\hat{z}_{\mathrm{c} . \mathrm{m} .}(\hat{t}[T+1]-\epsilon)=\frac{1}{N} \hat{v}[T]+O(\epsilon)$.

In summary, we obtain the following stochastic dynamics in tick time:

$$
\begin{aligned}
\hat{z}_{\text {c.m. }}[T+1]= & \hat{z}_{\text {c.m. }}[T]+c \hat{\tau}[T] \tanh \frac{\Delta \hat{p}[T]}{\Delta p^{*}} \\
& +\sqrt{\frac{\sigma^{2} \hat{\tau}[T]}{N}} \hat{\mu}[T]+\frac{1}{N} \hat{v}[T], \\
\hat{p}[T+1]= & \hat{z}_{\mathrm{c} . \mathrm{m} .}[T+1]+\frac{L_{\rho}^{*}}{2 \sqrt{N}} \hat{\xi}[T], \\
\Delta \hat{p}[T+1]= & \hat{p}[T+1]-\hat{p}[T],
\end{aligned}
$$

with Gaussian random number $\hat{\xi}[T]$ with unit variance. To be precise, $\hat{z}_{\text {c.m. }}[T] \equiv \lim _{\epsilon \rightarrow+0} \hat{z}_{\text {c.m. }}(\hat{t}[T]+\epsilon), \hat{p}[T] \equiv$ $\lim _{\epsilon \rightarrow+0} \hat{p}(\hat{t}[T]+\epsilon)$, and $\Delta \hat{p}[T] \equiv \lim _{\epsilon \rightarrow+0} \Delta \hat{p}(\hat{t}[T]+\epsilon)$. By eliminating the two variables $\left(\hat{z}_{\text {c.m. }}, \hat{p}\right)$ from Eq. (89), we obtain Eq. (80) as a single stochastic difference equation in the tick time.

\section{Solution}

The macroscopic dynamics of the price strongly depends on the balance between the strength of the trend-following effect and that of the zigzag noise. Here, we present the solutions of the financial Langevin equation depending on the strength of trend following using dimensional analysis. The price movement originating from trend-following behavior is estimated to be $c \tau^{*}$ (of price dimension). On the other hand, the amplitude of the zigzag noise is estimated to be $L_{\rho}^{*} / \sqrt{2 N}$ (of price dimension). Their balance is thus characterized by the dimensionless parameter $\tilde{c}$ defined by

$$
\tilde{c} \equiv \frac{c \tau^{*}}{L_{\rho}^{*} / \sqrt{2 N}}=\frac{c L_{\rho}^{*}}{\sigma^{2} \sqrt{2 N}} .
$$

Another dimensionless control parameter is the ratio $\Delta \tilde{p}^{*}$ between the average movement by the trend following $c \tau^{*}$ (of price dimension) and the saturation threshold against the market trend $\Delta p^{*}$ (of price dimension):

$$
\Delta \tilde{p}^{*} \equiv \frac{\Delta p^{*}}{c \tau^{*}} .
$$




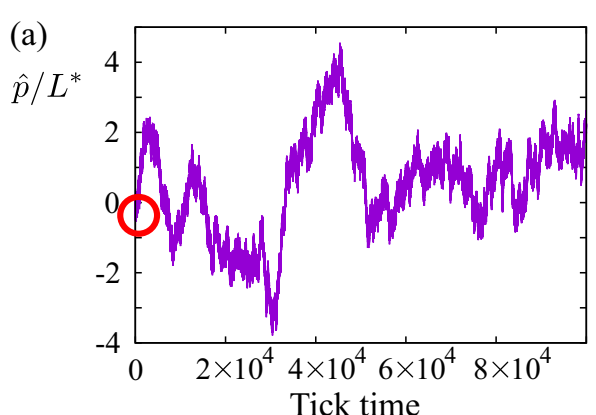

(Weak trend-following case)

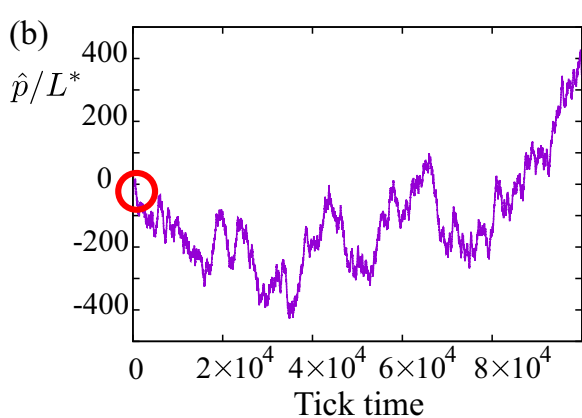

(Strong trend-following case)

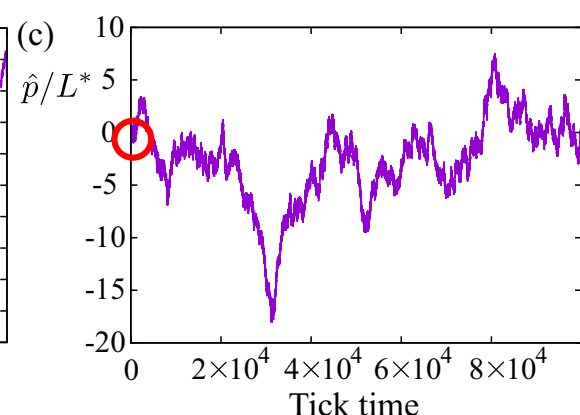

(Marginal trend-following case)

enlarge the circles


FIG. 12. Sample trajectories are plotted for (a) the weak trend-following case $\tilde{c}=0$, (b) the strong trend-following case $\left(\tilde{c}, \Delta \tilde{p}^{*}\right)=$ $(2.0,0.1)$, and (c) the marginal trend-following case $\left(\tilde{c}, \Delta \tilde{p}^{*}\right)=(0.5,2.5)$ for $N=100,10^{5}$ ticks, and the $\gamma$-distributed spread. All parameters are shared except for the trend-following parameters $\left(\tilde{c}, \Delta \tilde{p}^{*}\right)$. As can be seen from the figures, all trajectories seem to be the normal diffusion in the long timescale. The sample trajectories are enlarged 100 times [in the circles in panels (a)-(c)] as panels $\left(\mathrm{a}^{\prime}\right)-\left(\mathrm{c}^{\prime}\right)$, where the character of each trajectory can be seen. $\left(a^{\prime}\right)$ The price trajectory exhibits a zigzag behavior in the absence of trend following. ( $\left.b^{\prime}\right)$ The price keeps moving toward the same direction for a certain tick period because of the strong trend following. ( $\left.c^{\prime}\right)$ The price trajectory exhibits both zigzag behavior and trend following because both effects are in balance.

The set of dimensionless parameters $\left(\tilde{c}, \Delta \tilde{p}^{*}\right)$ governs the qualitative dynamics of the market price. For consistency with the empirical report [46], we focus on the case of $\Delta \tilde{p}^{*} \lesssim 1$ in this section, whereby the saturation of the hyperbolic function is valid (see Sec. VII I for the discussion on the case with $\left.\Delta \tilde{p}^{*} \gg 1\right)$. Here we introduce three classifications in terms of the strength of trend following:

(1) Weak trend-following case: $\tilde{c} \ll 1$.

(2) Strong trend-following case: $\tilde{c} \gg 1$.

(3) Marginal trend-following case: $\tilde{c} \sim 1$.

Sample trajectories are plotted in Fig. 12 to highlight the character of each case: For the weak trend-following case [Fig. 12(a)], the price tends to move upward and downward alternatively every tick because of the zigzag noise $\Delta \hat{\xi}$. For the strong trend-following case [Fig. 12(b)], the unidirectional movement of price is kept for a certain time period. For the marginal trend-following case [Fig. 12(c)], both zigzag and unidirectional movements randomly appear because both effects are in balance. As will be shown later in detail, the marginal case may be the most realistic, at least in our data set. We next study these qualitative characters through statistical analysis of price time series within the mean-field approximation.

\section{Weak trend-following case}

For the weak trend-following case $\tilde{c} \ll 1$, the trendfollowing effect is negligible compared with the zigzag noise: $\left|c \hat{\tau}[T] \tanh \left(\Delta \hat{p}[T] / \Delta p^{*}\right)\right| \ll|\Delta \hat{\xi}[T]|$. The master equation (72) can then be analytically solved in continuous time $t$. By ignoring the trend-following term, we integrate Eq. (72) with respect to $p$ and $\Delta p$ to obtain

$$
\begin{aligned}
\frac{\partial P_{t}\left(z_{\mathrm{c} . \mathrm{m} .}\right)}{\partial t}= & \frac{\sigma^{2}}{2 N} \frac{\partial^{2} P_{t}\left(z_{\mathrm{c} . \mathrm{m} .}\right)}{\partial z_{\mathrm{c} . \mathrm{m} .}^{2}}+\frac{1}{\tau^{*}} \int_{-\infty}^{\infty} d y w_{N}(y) \\
& \times\left[P_{t}\left(z_{\mathrm{c} . \mathrm{m} .}-y\right)-P_{t}\left(z_{\mathrm{c} . \mathrm{m} .}\right)\right]
\end{aligned}
$$

Given that $w_{N}(y)$ satisfies the scaling of the system size expansion (76), we apply the Kramers-Moyal expansion [13,16]

$$
\begin{aligned}
& \frac{1}{\tau^{*}} \int_{-\infty}^{\infty} d y w_{N}(y)\left[P_{t}\left(z_{\mathrm{c} . \mathrm{m} .}-y\right)-P_{t}\left(z_{\mathrm{c} . \mathrm{m} .}\right)\right] \\
& =\frac{2 \sigma^{2}}{L_{\rho}^{* 2}} \sum_{k=1}^{\infty} \frac{(-1)^{k}}{N^{k-1}} \frac{\alpha_{k}}{k !} \frac{\partial^{k} P_{t}\left(z_{\mathrm{c} . \mathrm{m} .}\right)}{\partial z_{\mathrm{c} . \mathrm{m} .}^{k}}
\end{aligned}
$$

with the $N$-independent Kramers-Moyal coefficient $\alpha_{k} \equiv$ $\int_{-\infty}^{\infty} d y y^{n} \tilde{w}(y)$. By taking the series up to the order of $N^{-1}$, we finally obtain the diffusion equation for the c.m. (94) as

$$
\frac{\partial P_{t}\left(z_{\mathrm{c} . \mathrm{m} .}\right)}{\partial t}=D(N) \frac{\partial^{2} P_{t}\left(z_{\mathrm{c} . \mathrm{m} .}\right)}{\partial z_{\mathrm{c} . \mathrm{m} .}^{2}}, \quad D(N) \equiv \frac{\sigma^{2}}{2 N}\left(1+\frac{2 \alpha_{2}}{L_{\rho}^{* 2}}\right)
$$


with the renormalized diffusion coefficient $D(N)$ up to the order of $N^{-1}{ }^{8}$ We note that $\alpha_{2}=L^{* 2}$ for the $\gamma$-distributed spread. The diffusion constant $D(N)$ decays for $N \rightarrow \infty$, which implies that the dynamics of the c.m. become slower as the number of traders increases. Given that the dynamics of price $\hat{p}$ coincide with those of the c.m. $\hat{z}_{\mathrm{c} . \mathrm{m}}$. for a long timescale, the diffusion of the price is also shown to be normal for a long timescale with the same diffusion coefficient $D(N)$ in real time $t$. The mean-squared displacement (MSD) based on real time $t$ is thus analytically obtained as

$$
\operatorname{MSD}(t) \equiv\left\langle[\hat{p}(t)-\hat{p}(0)]^{2}\right\rangle \sim 2 D(N) t,
$$

showing the normal diffusion for a long time.

We also study price movement at one-tick precision. For the weak trend-following case, the only relevant term in Eq. (80) is the zigzag noise $\Delta \hat{\xi}(T)$ for a short timescale. Price movement $\Delta \hat{p}$ then obeys the Gaussian distribution

$$
P(\Delta p) \approx \mathcal{N}\left(\Delta p ; \frac{L_{\rho}^{* 2}}{2 N}\right), \quad\left\langle\Delta \hat{p}^{2}\right\rangle \approx \frac{L_{\rho}^{* 2}}{2 N} .
$$

The autocorrelation function of the price movement $\Delta \hat{p}$ is also given by

$$
\begin{aligned}
C_{\Delta \hat{p}}[K] & \equiv \frac{\langle\Delta \hat{p}[T+K] \Delta \hat{p}[T]\rangle}{\left\langle\Delta \hat{p}[T]^{2}\right\rangle} \\
& \approx C_{\Delta \hat{\xi}}[K] \approx \begin{cases}1 & (K=0), \\
-1 / 2 & (K=1), \\
0 & (K \geqslant 2) .\end{cases}
\end{aligned}
$$

Interestingly, this property is consistent with an empirical fact that price movements typically exhibit zigzag behavior for a short timescale.

Here we discuss the origin of the strong negative correlation in terms of price movement. Remarkably, only the random noise $\hat{\zeta}[T]$ is dominant for a long time whereas only the zigzag noise $\Delta \hat{\xi}[T]$ is dominant for a short timescale. For $K \gg N$, indeed, we obtain

$$
\begin{aligned}
\hat{p}[T+K]-\hat{p}[T]= & \sum_{i=0}^{K-1}(\Delta \hat{\xi}[T+i]+\hat{\zeta}[T+i]) \\
= & \sqrt{\sqrt{\frac{L_{\rho}^{* 2}}{4 N}(\hat{\xi}[T+K-1]-\hat{\xi}[T-1])}} \\
& +\underbrace{\sum_{i=0}^{K-1} \hat{\zeta}[T+i]}_{O\left(N^{-1 / 2}\right)},
\end{aligned}
$$

which implies that the contribution by the zigzag noise $\hat{\xi}[T]$ is negligible compared with that of the random noise $\hat{\zeta}[T]$ [i.e., $\left.\sum_{i=0}^{K-1} \hat{\zeta}[T+i]=O\left(N^{-1} K^{1 / 2}\right) \gg O\left(N^{-1 / 2}\right)\right]$. Considering

\footnotetext{
${ }^{8}$ Introduction of a renormalized time $\tilde{t} \equiv t / N$ is also useful in clarifying the appropriate timescale at which the diffusive approximation holds, according to the original formulation of the system size expansion.
}

that the random noise $\hat{\zeta}[T]$ originates from the diffusion of the c.m., Eq. (98) implies that the macroscopic behavior of price is governed by the slow dynamics of the c.m. Even though the price movement at one-tick precision is much larger than that of the c.m., such movement is irrelevant to the macroscopic dynamics of the whole system. This is the origin of the strong negative correlation for price movement in this model for the weak trend-following case. To relieve such negative correlation, stronger trend following is necessary to induce the collective motion of the order book as discussed in Ref. [46]. We note that similar slow diffusion is observed in the conventional zero-intelligence order-book models [3840], with which the trend-following effect is not incorporated likewise.

We also note that the negative correlation (97) is also related to the slow diffusion of price for a short timescale. Indeed, the MSD for the tick time $K$ is given by

$$
\operatorname{MSD}[K]=\left\langle(\hat{p}[T+K]-\hat{p}[T])^{2}\right\rangle \approx \frac{L_{\rho}^{* 2}}{2 N}+2 D(N) \tau^{*} K
$$

within the mean-field approximation. This formula implies that the MSD is almost constant (i.e., no diffusion) for a short timescale $K \ll N$, while it is asymptotically linear (i.e., the normal diffusion) for a long timescale $K \gg N$.

Numerical comparison. Here, we examine the validity of our formulas through comparison with numerical results for the $\gamma$-distributed spread (see Sec. VC for the implementation).

(a) Transaction interval. We first check the statistics of the time interval between transactions $\hat{\tau}$. The mean transaction interval $\tau^{*} \equiv\langle\hat{\tau}\rangle$ is numerically plotted in Fig. 13(a), showing the quantitative agreement with the theoretical prediction (81) including the coefficient. In Fig. 13(b), we also numerically plot the probability distribution of $\hat{\tau}$ with scaling parameters for the horizontal and vertical axes, qualitatively showing the exponential tail for large $\hat{\tau}$. Here we have introduced a scaled transaction interval $\tilde{\tau} \equiv c_{\tau} \hat{\tau} / \tau^{*}$ and plotted the scaled probability distribution

$$
\tilde{P}(\tilde{\tau}) \equiv \frac{\tau^{*} P(\tau)}{Z_{\tau}} \sim e^{-\tilde{\tau}}(\tilde{\tau} \rightarrow \infty),
$$

with scaling parameters for the horizontal and vertical axes $c_{\tau}$ and $Z_{\tau}$, respectively. The coefficients $c_{\tau}$ and $Z_{\tau}$ were determined using the least-squares method to fit the exponential tail for each $N$. The numerical results imply the modification for the decay length $c_{\tau} \approx 1.6$, whereas the mean-field solution (81) predicts $c_{\tau}=1$. This implies that the mean-field solution (81) is not exact but is rather qualitatively correct for the probability distribution $P(\tau)$.

This factor modification $c_{\tau} \approx 1.6$ can be roughly understood from the viewpoint of the order statistics, as discussed in Ref. [46]. The mean-field approximation predicts the exponential interval distribution (81), which implies that the transaction obeys the exact Poisson process. As the numerics shows, however, the transaction obeys the Poisson process not exactly but only asymptotically. An explanation for this observation is that a transaction occurs as a pair of arrivals of both bid and ask quotes. Let us assume that the arrival of 
(a) $\tau^{*}$

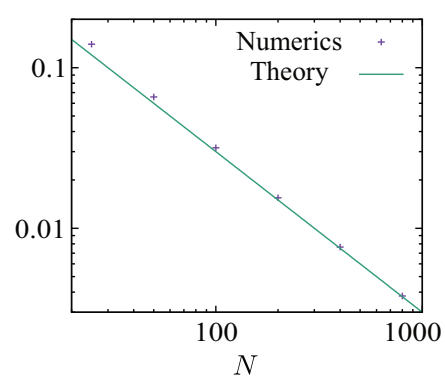

(e) $\left\langle\Delta \hat{p}^{2}\right\rangle / L^{* 2}$

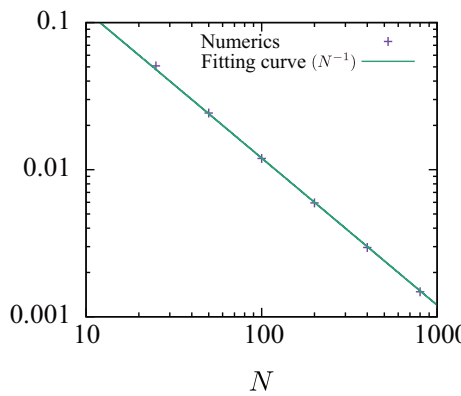

(b) $\tilde{P}(\tilde{\tau})$

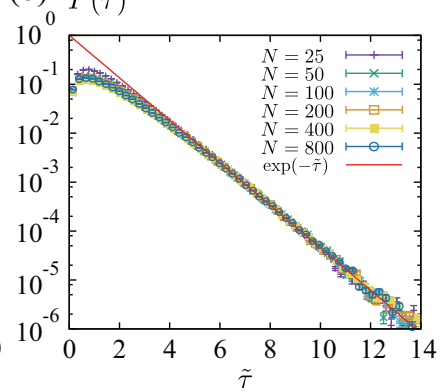

(f) $P(|\Delta \tilde{p}|)$

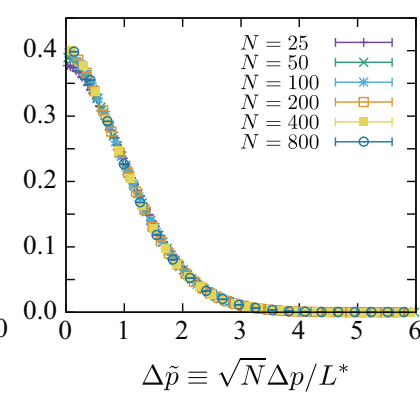

(c) $\operatorname{MSD}(t)$

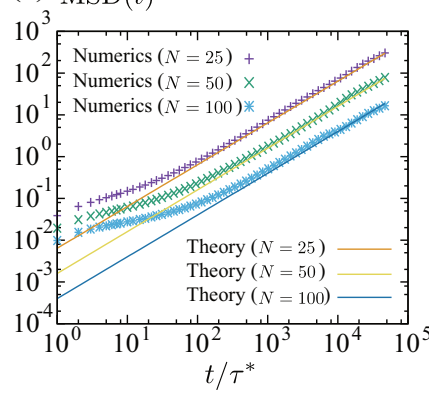

(g) $P(|\Delta \tilde{p}|)$

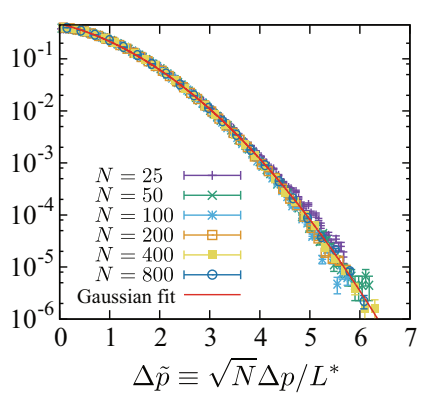

(d) $\operatorname{MSD}[K]$

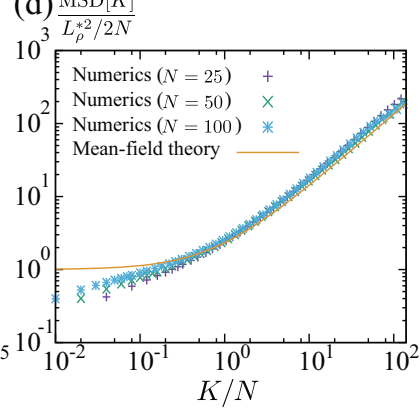

(h) $C_{\Delta \hat{p}}[K]$

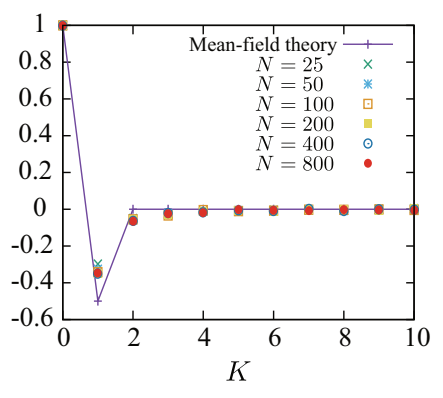

FIG. 13. Numerical study in the absence of trend following $c=0$. (a) Numerical mean transaction intervals $\tau^{*}$ and the theoretical line for various $N$. (b) Transaction interval distribution for various $N$ with an exponential guideline by scaling the horizontal and vertical axes. The scaled interval is given by $\tilde{\tau}=c_{\tau} \hat{\tau} / \tau^{*}$, where the fitting parameter $c_{\tau}$ for the decay time was estimated by the least-squares method for the tail as $c_{\tau}=1.34,1.49,1.56,1.58,1.62$, and 1.59 for $N=25,50,100,200,400$, and 800, respectively. (c) MSD plot based on real time $t$ for various $N$ with the theoretical lines, showing the normal diffusion for large $t$ but the slow diffusion $t \sim \tau^{*}$. (d) MSD plot based on tick time $K$ for various $N$ with the theoretical line, showing the normal diffusion for large $K$ but the slow diffusion for small $K$. (e) Variance of price difference $\Delta \hat{p}$ for various number of traders $N$ with a fitting curve of power-law exponent $N^{-1}$. (f) Plot of the peak of the PDF $P(\Delta \tilde{p})$ for the scaled price movement $\Delta \tilde{p} \equiv \sqrt{N} \Delta p / L^{*}$. (g) Log plot of the tail of the PDF $P(\Delta \tilde{p})$ with a Gaussian fitting curve $h(\Delta \tilde{p})$. (h) Autocorrelation function $C_{\Delta \hat{p}}[K]$ with tick time $K$, showing negative correlation at $K=1$. The simulation time was set to be $10^{5}$ ticks except for panels (a), (b), and (e)-(h), whereas the simulation time was set to be $10^{6}$ ticks for panels (c) and (d).

a bid (ask) quote at the transaction price obeys the Poisson statistics as $P\left(\tau_{B}\right)=e^{-\tau_{B} / \tau_{B}^{*}} / \tau_{B}^{*}\left[P\left(\tau_{A}\right)=e^{-\tau_{A} / \tau_{A}^{*}} / \tau_{A}^{*}\right]$. Any transaction is assumed to occur when both bid and ask quotes arrive at the transaction price. We then make an approximation that $\hat{\tau} \approx \max \left\{\hat{\tau}_{B}, \hat{\tau}_{A}\right\}$ and $\tau_{B}^{*}=\tau_{A}^{*}$. On the basis of the orders statistics [55], we obtain

$$
P(\tau) \approx 1-\left(1-e^{-3 \tau / 2 \tau^{*}}\right)^{2} \sim e^{-3 \tau / 2 \tau^{*}},
$$

where the fitting parameter was determined by the consistency condition for the average interval as $\langle\hat{\tau}\rangle=\tau^{*} \Longleftrightarrow \tau_{B}^{*}=$ $3 \tau^{*} / 2$. We thus obtain the modification factor $c_{\tau}=3 / 2$ as an approximation.

We note that the transaction interval is not under the influence of the trend-following effect. This statistical characteristic of the transaction interval is therefore shared for any parameter set of $\left(\tilde{c}, \Delta \tilde{p}^{*}\right)$.

(b) MSD. Our theoretical prediction on the MSD is numerically examined for analyses based on both real time $t$ and tick time $K$. We first numerically check the MSD (95) based on the real time $t$ in Fig. 13(c). This figure shows the quantitative agreement with our theoretical formula (95) without fitting parameters. We also check the MSD based on the tick time $K$ in Fig. 13(d), showing a quantitative agreement with the theoretical prediction (99) for $K \gg 1$. For small $K \sim 1$, the agreement is not perfect between the numerical data and the theoretical line, but the slowness of the diffusion is qualitatively observed as predicted in the mean-field solution (99).

(c) Price movement. The dependence of the variance of price movement is examined in Fig. 13(e) on the number of traders $N$. We numerically obtained $\left\langle\Delta \hat{p}^{2}\right\rangle \approx$ $C_{\left\langle\Delta \hat{p}^{2}\right\rangle}\left(L_{\rho}^{* 2} / 2 N\right)$ with modification factor $C_{\left\langle\Delta \hat{p}^{2}\right\rangle} \approx 0.4$. Although there is a discrepancy in terms of the factor $C_{\left\langle\Delta \hat{p}^{2}\right\rangle}$, the mean-field solution (96) qualitatively works well for the variance of price movement. We also checked the PDF $P(|\Delta \tilde{p}|)$ of the scaled price movement $\Delta \tilde{p} \equiv \sqrt{N} \Delta p / L^{*}$ [Figs. 13(f) and 13(g) for the peak and tail of the PDF, respectively]. In Fig. 13(g), we also show a Gaussian-type fitting curve $h(\Delta \tilde{p})=\exp \left(-h_{0}^{*}-h_{1}^{*} \Delta \tilde{p}-h_{2}^{*} \Delta \tilde{p}^{2}\right)$ for the tail with parameters $h_{0}^{*}=0.75 \pm 0.05, h_{1}^{*}=0.54 \pm 0.04$, and $h_{2}^{*}=0.238 \pm 0.006$. These figures suggest that the PDF of the price movement has a Gaussian tail, which is qualitatively consistent with the theoretical prediction (96) $\left(h_{1}^{*}=0\right.$ and $\left.h_{2}^{*}=1 / 6\right)$.

(d) Autocorrelation. The autocorrelation function $C_{\Delta \hat{p}}[K]$ is checked in Fig. 13(h), which supports the qualitative consistency between the theory (98) and the numerical results in terms of the negative correlation at $K=1$ tick. This negative correlation implies that the price time series exhibits the zigzag behavior in the absence of the trend-following effect. Indeed, the probability of $\Delta \hat{p}[T+1] \Delta \hat{p}[T]<0$ is theoretically $2 / 3=66.6 \ldots \%$ for the mean-field model, which is 
TABLE II. Table of the probabilities of the same successive sign and different sign for the price movement time series $\{\Delta \hat{p}[T]\}_{T}$. We numerically obtained the probability that the next price movement $\Delta \hat{p}[T+1]$ has the same (different) sign as (from) that of the previous price movement $\Delta \hat{p}[T]$ for $N=100$. (a) For the weak trend-following case $\tilde{c}=0$, the probability of taking a different sign is higher than that of taking the same sign, implying the zigzag motion of the price movement. (b) For the strong trend-following case $\left(\tilde{c}, \Delta \tilde{p}^{*}\right)=(2.0,0.1)$, the probability of taking the same successive sign is much higher than that of taking a different sign, implying the ballistic motion of the price movement. (c) For the marginal trend-following case $\left(\tilde{c}, \Delta \tilde{p}^{*}\right)=(0.5,2.5)$, the probability of taking a different sign is slightly higher than that of taking the same sign. (d) We also obtained the probabilities from the real price time series in our data set, showing that the probability of taking a different sign is slightly higher than that of taking the same sign. For simplicity, we omitted zero, such as $\Delta p[T]=0$, during the data analysis of the real price movement time series $\{\Delta \hat{p}[T]\}_{T}$. This table implies that the marginal trend-following case is consistent with the real price time series and is the most realistic at least for our data set.

\begin{tabular}{lcc}
\hline Case & Same sign & Different sign \\
\hline (a) Weak trend-following case & 0.389 & 0.611 \\
(b) Strong trend-following case & 0.949 & 0.051 \\
(c) Marginal trend-following case & 0.480 & 0.520 \\
(d) Real price time series & 0.479 & 0.521 \\
\hline \hline
\end{tabular}

considerably higher than $50 \%$ (i.e., the pure random walks). This result is also qualitatively consistent with the numerical result (approximately 61\%) as shown in Table II.

The theoretical prediction for the probability of $\Delta \hat{p}[T+$ 1] $\Delta \hat{p}[T]<0$ can be obtained as follows: let us introduce three Gaussian random variables $\hat{x}, \hat{y}, \hat{z}$ with unit variance and investigate $\hat{w} \equiv \hat{y}-\hat{x}$ and $\hat{u} \equiv \hat{z}-\hat{y}$. To analyze the probability of the successive same sign for the mean-field model (80), it is sufficient to study the probability of taking the same sign for $\hat{w}$ and $\hat{u}$ as

$$
\begin{aligned}
& P(\hat{w}>0 \cap \hat{u}>0)+P(\hat{w}<0 \cap \hat{u}<0) \\
& \quad=2 P(\hat{w}>0 \cap \hat{u}>0)=\int_{0}^{\infty} \operatorname{dwdu} P(w, u) .
\end{aligned}
$$

Here we obtain

$$
\begin{aligned}
P(\hat{w}>0 \cap \hat{u}>0) & \int_{0}^{\infty} d w d u \int_{-\infty}^{\infty} d x d y d z \delta(w-y+x) \\
& \times \delta(u-z+y) \frac{e^{-\left(x^{2}+y^{2}+z^{2}\right) / 2}}{(2 \pi)^{3 / 2}} \\
= & \int_{0}^{\infty} d w d u \int_{-\infty}^{\infty} \frac{d z}{(2 \pi)^{3 / 2}} \exp \left[-\frac{1}{2}(z-u-w)^{2}\right. \\
& \left.-\frac{1}{2}(z-u)^{2}-\frac{1}{2} z^{2}\right]=\frac{1}{6}
\end{aligned}
$$

The probability of successive same sign for the mean-field model (80) is thus given by $1 / 3$. The probability of $\Delta \hat{p}[T+$ $1] \Delta \hat{p}[T]<0$ is then given by $2 / 3$.

\section{Strong trend-following case}

The strong trend-following case $\tilde{c} \gg 1$ is also analytically tractable, whereby the trend-following term is dominant such that $\left|c \hat{\tau}[T] \tanh \left(\Delta \hat{p}[T] / \Delta p^{*}\right)\right| \gg|\max \{\Delta \hat{\xi}[T], \hat{\zeta}[T]\}|$. Here we assume that the saturation threshold is sufficiently small such that $\Delta \tilde{p}^{*} \ll 1$. This condition simplifies the following analysis because the hyperbolic function can be approximated as the signature function, such that $\tanh \left(\Delta \hat{p}[T] / \Delta p^{*}\right) \approx \operatorname{sgn}(\Delta \hat{p}[T])$. Price movement is then governed by the first term on the rhs of Eq. (80), which approximately leads the exponential distribution,

$$
P(|\Delta p|) \propto e^{-|\Delta p| / \kappa},
$$

with decay length $\kappa$ for $|\Delta p| \rightarrow \infty$. The decay length is given by the mean movement originating from the trend following as $\kappa=c \tau^{*}$ within the mean-field approximation (81). By applying the improved mean-field approximation (101), a more consistent coefficient $\kappa=2 c \tau^{*} / 3$ is obtained with the numerical result which follows. The trend-following effect plays similar roles to momentum inertia in physics, which are reflected in the autocorrelation function and the MSD plot as shown numerically in the next paragraph.

Numerical comparison. Numerical characters are studied here for the strong trend-following case under the parameter set $\left(\tilde{c}, \Delta \tilde{p}^{*}\right)=(2.0,0.1)$. We first study the price movement distribution $P(|\Delta p|)$. In Fig. 14(a), the price movement distribution is plotted by scaling the horizontal and vertical axes,

$$
\tilde{P}(|\Delta \tilde{p}|)=\frac{\kappa P(|\Delta p|)}{Z_{\Delta \tilde{p}}} \sim e^{-|\Delta \tilde{p}|},
$$

which qualitatively shows the exponential tail for the scaled price movement $\Delta \tilde{p} \equiv \Delta p / \kappa$. Here the scaling parameters $\kappa$ and $Z_{\Delta \tilde{p}}$ were determined by the least-squares method for the tail. The mean-field solution (81) and the improved mean-field solution (101) predicts $\kappa=c \tau^{*}$ and $\kappa=2 c \tau^{*} / 3$, respectively. These theoretical predictions are qualitatively consistent with the numerical estimation $\kappa \approx 0.64 c \tau^{*}$.

We next investigate the autocorrelation function $C_{\Delta \hat{p}}[K]$ of the price difference $\Delta \hat{p}$ based on the tick time $K$ in Fig. 14(b) by scaling the horizontal line. For our parameter sets, the numerical result implies that the autocorrelation function can be written as

$$
C_{\Delta \hat{p}}[K] \approx \begin{cases}1 & (K=1), \\ \frac{1}{Z_{\mathrm{AC}}} e^{-K / \tau_{\mathrm{AC}}} & (K \geqslant 2),\end{cases}
$$

with fitting parameters $\tau_{\mathrm{AC}}$ and $Z_{\mathrm{AC}}$. This autocorrelation suggests that the strong trend following keeps unidirectional price movements for a certain time interval. Indeed, the probability of $\Delta \hat{p}[T] \Delta \hat{p}[T+1]>0$ is much higher than $50 \%$ under this condition as shown in Table II. In addition, the numerical MSD plot in Fig. 14(c) shows the rapid diffusion (almost ballistic motion $K^{2}$ ) for a short time and the normal diffusion for a long time.

\section{Marginal case}

The most complex case is the marginal case $\tilde{c} \sim 1$, where both trend-following effect and zigzag noise contribute to the price movement as $\left|c \hat{\tau}[T] \tanh \left(\Delta \hat{p}[T] / \Delta p^{*}\right)\right| \sim|\Delta \hat{\xi}[T]|$. 

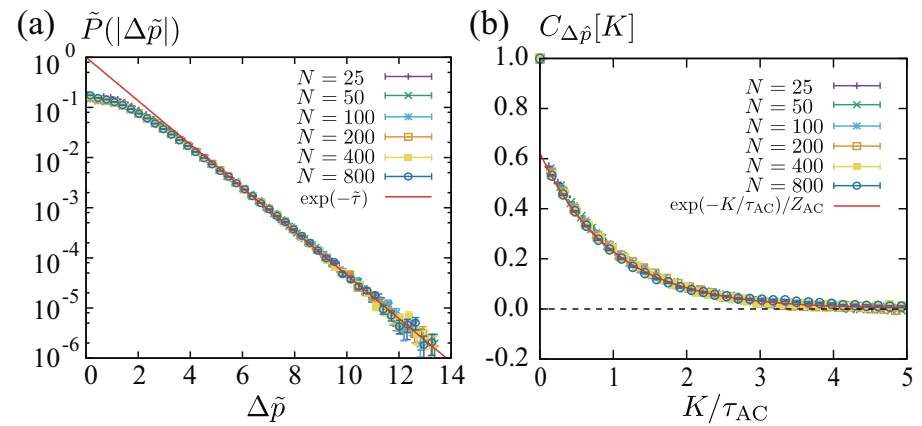

(c) $\operatorname{MSD}[K]$

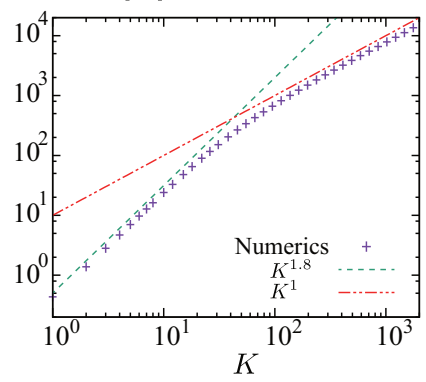

FIG. 14. Numerical study for the strong trend-following case $\tilde{c} \gg 1$. We adopted $\left(\tilde{c}^{*}, \Delta \tilde{p}^{*}\right)=(2.0,0.1)$ as the trend-following parameters. (a) Price movement distribution for various $N$ by scaling both horizontal and vertical axes, qualitatively showing the exponential law (104). The least-squares method numerically estimates the decay length as $\kappa / c \tau^{*}=0.74,0.68,0.65,0.64,0.64$, and 0.64 for $N=25,50,100,200,400$, and 800, respectively. (b) Autocorrelation function $C_{\Delta \hat{p}}[K]$ with tick time $K$, showing the positive correlation with exponential decay. The fitting parameters were estimated to be $Z_{\mathrm{AC}}=0.62$ and $\tau_{\mathrm{AC}}=16.4,14.2,13.1,12.8,12.2$, and 12.6 for $N=25,50,100,200,400$, and 800 , respectively. (c) Numerical MSD plot for $N=50$, showing a rapid diffusion of exponent $K^{1.8}$ (almost the ballistic motion of exponent $K^{2}$ ) for a short time and a normal diffusion of exponent $K^{1}$ for a long time. The simulation time was set to be $10^{5}$ ticks for panels (a) and (b), whereas the simulation time was set to be $10^{6}$ ticks for panel (c).

While both the trend-following term $c \hat{\tau} \tanh \left(\Delta \hat{p} / \Delta p^{*}\right)$ and the random noise term $\Delta \hat{\xi}$ are relevant under this condition, the main contribution to the price movement tail originates from the trend-following term because the former yields an exponential tail while the latter yields a Gaussian tail. We thus obtain the exponential tail (104) for the price movement for the marginal case. This theoretical conjecture is to be validated numerically below.

Numerical comparison. We studied the marginal case for the parameter set $\left(\tilde{c}, \Delta \tilde{p}^{*}\right)=(0.5,2.5)$. In Fig. 15(a), we plot the price movement distribution by scaling both horizontal and vertical axes as Eq. (105). We thus qualitatively obtain the exponential-law tail (105) for the price movement.

In Fig. 15(b), we also studied the autocorrelation function $C_{\Delta \hat{p}}[K]$ on tick time $K$ through both numerical simulation (points) and empirical data analysis (solid line) of the real time series. This figure shows the slight negative correlation around $K=1$, which is qualitatively consistent with the empirical result in our data set. This result also implies that the price time series exhibits a slight zigzag behavior for a certain tick period. This theoretical implication was validated by analyzing the probability of $\Delta \hat{p}[T] \Delta \hat{p}[T+1]<0$ as summarized in Table II. Table II shows the quantitative consistency between the marginal trend-following case and the real price time series.

We also discuss the behavior of the MSD in Figs. 15(c) and 15(d), which shows both slow and rapid diffusions dependently of the chosen parameters. For example, we set the parameters $\left(\tilde{c}, \Delta \tilde{p}^{*}\right)=(0.5,2.5)$ and $\left(\tilde{c}, \Delta \tilde{p}^{*}\right)=(0.86,1.43)$ for Figs. 15(c) and 15(d), respectively. In Fig. 15(c), the MSD plot exhibits a slightly slow diffusion for a short time and a normal diffusion for a long time. In Fig. 15(d), on the other hand, the MSD plot exhibits a slightly rapid diffusion with the Hurst exponent $H=0.65$ for a short time and the normal diffusion for a long time. We thus conclude that our HFT model can reproduce a variety of diffusions by adjusting the trend-following parameters.

\section{DISCUSSION}

We here discuss implications of our theory to understand various topics intensively.

\section{A. Comparison with real data set}

Here we provide a detailed comparison between the empirical facts and the aforementioned theoretical predictions as follows: As for the order-book profile $f_{A}(r)$, the validity of the formula (70) was examined by analyzing the daily average order book in Ref. [46]. The exponential tail for the time interval distribution $P(\tau) \sim e^{-\tau / \tau^{*}}$ was studied in Ref. [56] by removing the nonstationary property of the time series. The price movement was reported to obey the exponential law $P(|\Delta p|) \sim e^{-|\Delta p| / \kappa}$ in Ref. [46] by removing the nonstationary property of the time series. The price time series tended to exhibit zigzag behaviors, which were reflected in the negative autocorrelation function $C_{\Delta \hat{p}}[K]$ around $K=1$ [see Fig. $15(\mathrm{c})]$ and the probability of $\Delta \hat{p}[T] \Delta \hat{p}[T+1]<0$ (i.e., taking different signs) slightly over 50\% (see Table II). All these characters are consistent with our theoretical prediction for the marginal trend-following case (see Table III for a summary of the comparison). The HFT model presented here can show precise agreements with these empirical facts. Considering that the market was not volatile in our data set, we concluded that our HFT model can describe the FX market well, at least during the stable period. The description of unstable markets is out of the scope of this paper and is a next interesting problem for future studies.

\section{B. Validity of mean-field approximation}

We have numerically validated the mean-field theory. The LO solution (63) quantitatively describes the order-book profile (66) with high precision and the NLO solution (61) qualitatively describes the price movement (80). Here we discuss possible reasons for the excellent performance of the mean-field approximation for the trend-following HFT model considering a common sense in the field of statistical physics.

The mean-field approximation is expected to be invalid for low-dimensional physical systems because two-body correlations do not disappear between colliding pairs for a long time. Colliding particles are not allowed to be separated far from each other because of the continuity of paths and the low-dimensional space geometry. For one-dimensional 


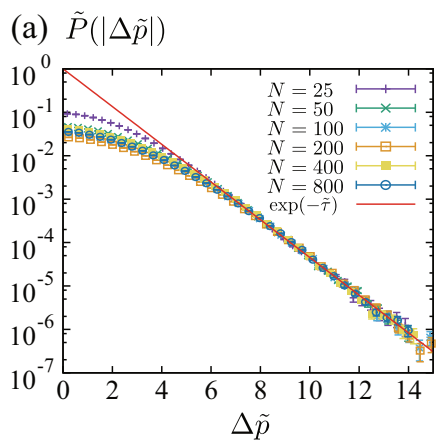

(b) $C_{\Delta \hat{p}}[K]$



(c) $\operatorname{MSD}[K]$

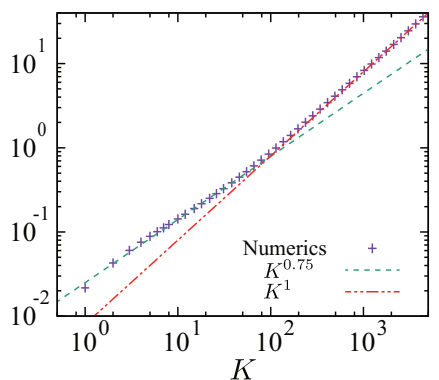

(d) $\operatorname{MSD}[K]$

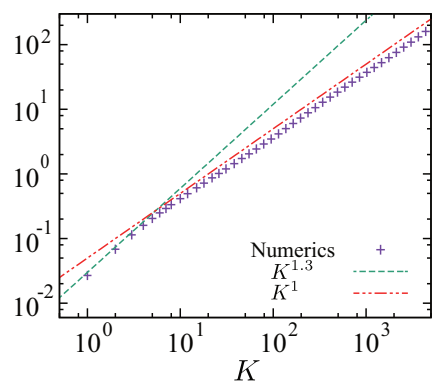

FIG. 15. Numerical study for the marginal case $\tilde{c} \sim 1$. (a) The price movement distribution $\Delta p$ by scaling the horizontal and vertical axes. (b) Autocorrelation function $C_{\Delta \hat{p}}[K]$ based on tick time $K$ (points), showing the negative correlation around $K=1$. This numerical result was consistent with the empirical result obtained from our data set (solid line). (c) MSD plot under the parameters $\left(\tilde{c}, \Delta \tilde{p}^{*}\right)=(0.5,2.5)$, showing a slightly slow diffusion. (d) MSD plot under the parameters $\left(\tilde{c}, \Delta \tilde{p}^{*}\right)=(0.86,1.43)$, showing a slightly rapid diffusion with the Hurst exponent $H=0.65$. The simulation time was set to be $10^{5}$ ticks for panels (a) and (b), whereas the simulation time was set to be $10^{6}$ ticks for panels (c) and (d).

Hamiltonian systems with hard-core interactions, for example, any particle successively collides with fixed neighboring particles and the two-body correlations remain forever. The mean-field approximation is therefore shown to be valid only for high-dimensional systems, at least for several concrete setups. From this viewpoint, the precise agreement is not trivial between the mean-field solution (66) and the numerical result.

In contrast, the continuity of the path is absent due to requotation jumps, although our model is a one-dimensional system. There is no restriction on the combination of possible transaction pairs because the transaction rule (17) compulsorily separates the transaction pairs after their collision. In the $N \rightarrow \infty$ limit, in addition, transactions between the same pair traders become rare (i.e., the probability of successive transactions between the same pair decays as the order of $N^{-2}$ ), which implies quick disappearance of the two-body correlation between transaction pairs for $N \rightarrow \infty$. This is our conjecture to validate the mean-field approximation for this model. If this conjecture is correct, kinetic-like descriptions might be valid for various agent-based systems, if agents are separated compulsorily to avoid successive interactions between the same pairs.

\section{Nonstationary property for price movements: Power-law behavior}

Financial markets are known to statistically exhibit strong nonstationary properties, such as intraday activity patterns. Here, we discuss the impact of such nonstationary properties on the price movements and its relation to the celebrated power-law behavior for a long time.
Our theoretical model implies that the exponential law (104) for the price movement is the basic statistical property. This property was shown to be consistent with the real price movement in Ref. [46] for a short time, by removing the nonstationary property in terms of the decay length $\kappa$. The decay length $\kappa$ is related to the number of traders $N$ and the strength of the trend following $c$, both of which are expected to have nonstationary properties. Indeed, the number of traders $N$ exhibits a trivial but strong nonstationary property with a correlation with the decay length (see also the Supplemental Material of Ref. [46]).

To illustrate this characteristic, let us analyze the statistical relation between the mean absolute price movement $\langle|\Delta \hat{p}|\rangle$ and the number of HFTs $N$ in our data set. We measured $\langle|\Delta \hat{p}|\rangle$ as a representative of the market volatility for a short time and studied its correlation with $N$ every two hours in Fig. 16(a). Spearman's rank correlation coefficient was 0.63 between $\langle|\Delta \hat{p}|\rangle$ and $1 / N$. This result implies that the market volatility is relatively small when $N$ is large, which is qualitatively consistent with our theoretical prediction of $\langle|\Delta \hat{p}|\rangle \approx$ $\kappa \sim 1 / N^{\beta}$ (e.g., $\beta=1$ if parameters are time constant other than $N)$. The regression analysis between $\log \langle|\Delta \hat{p}|\rangle$ and $\log N$ implies $\beta=0.86 \pm 0.1$ as shown in Fig. 16(b). We also note that both $\langle\Delta \hat{p}\rangle$ and $1 / N$ had a tendency to become large during inactive hours of the EBS market [Fig. 16(c)].

The nonstationary property of the market volatility is related to the power-law behavior of the price movement for a long time. In Ref. [46], the decay length $\kappa$ is shown to have a power-law distribution $P(\kappa) \propto \kappa^{-\alpha-1}$, which implies the power-law price movement for a long time as the

TABLE III. Comparison between the empirical facts of the EBS market and our theoretical prediction.

\begin{tabular}{lllll}
\hline \hline Case & $P(|\Delta p|)$ & $P(\tau)$ & $C_{\Delta p}[K]$ & Prob. of diff. sign \\
\hline (a) Weak trend-following case & Gaussian & Exponential & Strongly negative at $K=1$ & around 60\% \\
(b) Strong trend-following case & Exponential & Exponential & Strongly positive & less than 10\% \\
(c) Marginal trend-following case & Exponential & Exponential & Slightly negative around $K=1$ & around 52\% \\
(d) Empirical facts & Exponential & Exponential & Slightly negative around $K=1$ & around 52\% \\
\hline \hline
\end{tabular}


(a)

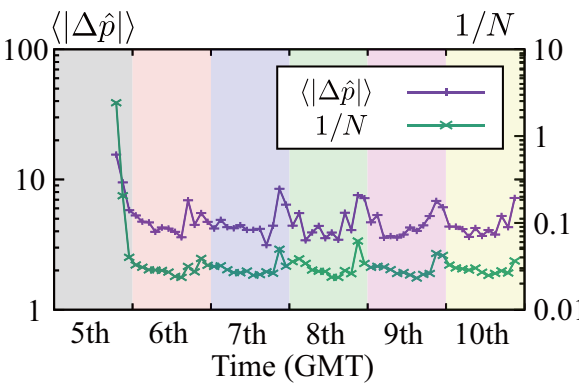

(b) $\langle|\Delta \hat{p}|\rangle$

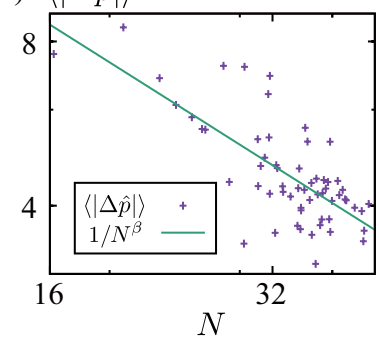

(c) $\langle|\Delta \hat{p}|\rangle$



FIG. 16. Empirical characteristics of the short-time market volatility $\langle|\Delta \hat{p}|\rangle$. (a) Time series of $\langle|\Delta \hat{p}|\rangle$ and the inverse number of HFTs $1 / N$. Both $\langle|\Delta \hat{p}|\rangle$ and $1 / N$ had a tendency to take large values during inactive hours of the EBS market, such as the time region just after the market opening (the 5th 18:00-22:00 GMT) and the end of the New York working hours (20:00-22:00 GMT). This figure exhibits the correlation between $\langle|\Delta \hat{p}|\rangle$ and $1 / N$ with Spearman's rank correlation coefficient of 0.63 . (b) Scattering plot between $\langle|\Delta \hat{p}|\rangle$ and $N$ in the log$\log$ scale. Regression analysis between $\log \langle|\Delta \hat{p}|\rangle$ and $\log N$ implies a power-law (almost linear) relation $\langle|\Delta \hat{p}|\rangle \propto 1 / N^{\beta}$ with $\beta=0.86 \pm 0.1$. In this analysis, we excluded the two samples after the market opening (the 5th 18:00-24:00 GMT) as outliers. (c) Intraday patterns are studied for $\langle|\Delta \hat{p}|\rangle$ and $N$. We took the averages of $\langle|\Delta \hat{p}|\rangle$ and $N$ conditional on two-hourly intraday time zones from Tuesday to Thursday, with the arrow-type legends showing the working hours for Tokyo (0:00-9:00 GMT), London (8:00-17:00 GMT), and New York (13:00-22:00 GMT). This figure shows that both $\langle|\Delta \hat{p}|\rangle$ and $1 / N$ tended to take large values during the end of the New York working hours (20:00-22:00 GMT).

superposition of the short-time exponential distribution,

$$
P_{\text {long }}(\geqslant|\Delta p|)=\int d \kappa P(\kappa) P_{\text {short }}(\geqslant|\Delta p|) \sim|\Delta p|^{-\alpha},
$$

with the complementary cumulative price movement distribution $P_{\text {long }}(\geqslant|\Delta p|)$ and $P_{\text {short }}(\geqslant|\Delta p|) \sim e^{-|\Delta p| / \kappa}$. This result is consistent with previous empirical research [23-26]. We thus concluded that both exponential law and power law can consistently coexist at least in our data set.

We here note that the FX market in our data set was rather stable without any external shocks. While the exponential law was essential for a short time in our data set, we do not deny the possibility that the power law may be essential even for a short time for unstable markets under external shocks. In our view, there would exist essentially different structures in unstable markets and it would be interesting to study the statistics of traders' behavior in these markets under financial crisis in a future investigation.

\section{Nonstationary property for transaction interval: Power-law behavior}

In terms of the transaction interval, our theory predicts that the exponential law (81) is essential rather than the power law. This result is consistent with the previous report in Ref. [56], showing that the exponential law is essential for a short time but its superposition leads to the power-law behavior of the transaction interval for a long time.

\section{E. Nonstationary property for order-book dynamics: Stability of the order-book profile}

We have discussed that both price movement and transaction interval are quite sensitive to nonstationary properties of the market. On the other hand, the average order-book profile $f_{A}(r)$ is relatively insensitive to such nonstationary properties, in contrast to the price movement and transaction interval. Indeed, the average order-book profile $f_{A}(r)$ is independent of the trend-following property $\tilde{c}$. In addition, the order-book profile shows a convergence for $N \rightarrow \infty$, such that $\lim _{N \rightarrow \infty} f_{A}(r)$ is an $L^{2}$ function, which implies that large variation of $N$ does not have an impact on the average order-book profile.

A similar insensitivity does not exist for the price movement and transaction interval. Indeed, they exhibit a strong divergence for $N \rightarrow \infty$ as $\lim _{N \rightarrow \infty} P(|\Delta p|)=\delta(|\Delta p|)$ and $\lim _{N \rightarrow \infty} P(\tau)=\delta(\tau)$, which implies that there is a significant impact of large variations of $N$ on their statistics.

In this sense, the average order-book profile is a stable quantity to measure under nonstationary processes, whereas the price movement and transaction interval are unstable quantities. Our theory provides insight on the sensitivity of measured quantities to the nonstationary nature of the market. We believe that developing systematic methods to remove such nonstationary characteristics is the key to understanding not only the origin of power laws in finance, but also the essence of market microstructure.

\section{F. More is different: $N=2$ vs $N \gg 1$}

One of the most interesting features in statistical physics lies in the fact that many-body systems can exhibit essentially different characteristics compared to few-body systems, such as critical phenomena and collective motion. Though the current HFT model does not exhibit critical phenomena, an essential difference can be shown between the cases of $N=2$ and $N \gg 1$. To illustrate this point, let us consider the case of $\tilde{c}=0$ without trend following. Our theory is applicable to solve the case of $N=2$ exactly, which leads to the same solution presented in Ref. [36]. The price movement is then predicted to obey the exponential law even without trend following, which is qualitatively different from the Gaussian law (96) for $N \rightarrow \infty$. This difference appears because the dynamics of the c.m. are not sufficiently slow for $N=2$. For $N=2$, indeed, one can show the absence of the zigzag noise term $\Delta \hat{\xi}[T]$ in the financial Langevin equation (80), which leads to the dominance of the random exponential noise $\hat{\zeta}[T]$. For $N \gg 1$, on the other hand, the random noise $\hat{\zeta}[T]$ is negligibly small due to the slow c.m. dynamics, and the trend-following effect becomes necessary to explain the exponential price movements statistics. The model presented 
here thus exhibits essentially different characteristics as the number of traders increases.

\section{G. Does the trend-following effect break the random walk hypothesis?}

Seemingly, the trend-following effect is strongly contradictory to the conventional assumption of the random walk hypothesis. Our analysis however implies that the situation is not so simple: In the absence of trend following, the market price exhibits the strong zigzag behavior, which is far from the pure random walks. By adjusting the strength of the trend following appropriately (i.e., the marginal trend-following case), on the other hand, the zigzag behavior is somewhat relieved and the market price time series approaches the random walks. In this sense, the trend-following strategy might originate from the rational behavior of HFTs to equilibrate the strategies among traders. It would be interesting to pursue the origin of trend-following behavior from economical viewpoints as future studies.

We also note that the real price time series exhibits slightly zigzag behaviors (i.e., the negative autocorrelation and the tendency for price movement to take different sign), which are consistent with our HFT model for the marginal trendfollowing case. These different characters from the pure random walks are well known in finance and are obviously applicable to predict the direction of price movement in the one-tick future. It is not easy however to make profits over the market spread (i.e., the difference between the market best bid and ask prices) by utilizing only these properties. While the real price time series slightly deviates from the pure random walks, it is not obvious whether these characteristics provide easy opportunities to statistically make profits. Making profits requires us to predict price movements beyond the market spread, which is beyond the scope of this paper, but is an interesting potential topic for a future study.

\section{H. Possible generalization 1: Multiple-tick trend-following random walks and the PUCK model}

In this paper, we have addressed the trend-following HFT model with one-tick memory. It is straightforward to generalize the one-tick memory model toward a multiple-tick memory model, such that

$$
\begin{aligned}
\left\langle\Delta \hat{z}_{i}[T]\right\rangle_{\Delta \hat{p}_{\mathrm{EMA}}[T-1]=\Delta p_{\mathrm{EMA}}} & =c \tanh \frac{\Delta p_{\mathrm{EMA}}}{\Delta p^{*}}, \\
\Delta \hat{p}_{\mathrm{EMA}}[T] & \equiv \sum_{K=0}^{\infty} \frac{e^{-K / \tau_{\mathrm{EMA}}}}{Z_{\mathrm{EMA}}} \Delta \hat{p}[T-K],
\end{aligned}
$$

where $\Delta \hat{p}_{\text {EMA }}[T-1]$ is the exponential moving average for the price movements $\{\Delta \hat{p}[T]\}_{T}$ with decay time $\tau_{\mathrm{EMA}}$ and renormalization constant $Z_{\mathrm{EMA}} \equiv 1 /\left(1-e^{-1 / \tau_{\mathrm{EMA}}}\right)$, and $\langle\ldots\rangle_{\Delta \hat{p}_{\mathrm{EMA}}[T-1]=\Delta p_{\mathrm{EMA}}}$ is the ensemble average conditional on $\Delta \hat{p}_{\text {EMA }}[T-1]=\Delta p_{\text {EMA }}$. In the authors' view, this model is more realistic because such an exponential moving average is a popular strategy among HFTs according to a detailed regression analysis for trend following [57]. We then obtain a generalization of the financial Langevin equation as

$$
\Delta \hat{p}[T+1]=c \hat{\tau}[T] \tanh \frac{\Delta \hat{p}_{\mathrm{EMA}}[T]}{\Delta p^{*}}+\Delta \hat{\xi}[T]+\hat{\zeta}[T] .
$$

The generalized financial Langevin equation (109) is equivalent to the potentials of unbalanced complex kinetics (PUCK) model [29], previously introduced on the basis of price time-series analyses. Here we use an identity

$$
\begin{aligned}
\Delta \hat{p}_{\mathrm{EMA}}[T] & =\frac{e^{1 / \tau_{\mathrm{EMA}}}}{Z_{\mathrm{EMA}}}\left\{\hat{p}[T+1]-\hat{p}_{\mathrm{EMA}}[T+1]\right\}, \\
\hat{p}_{\mathrm{EMA}}[T] & \equiv \sum_{K=0}^{\infty} \frac{e^{-K / \tau_{\mathrm{EMA}}}}{Z_{\mathrm{EMA}}} \hat{p}[T-K]
\end{aligned}
$$

for the exponential moving averages $\Delta \hat{p}_{\mathrm{EMA}}[T]$ and $\hat{p}_{\text {EMA }}[T]$, which leads to the PUCK model

$$
\begin{aligned}
\hat{p}[T+1]-\hat{p}[T]= & -\left.\frac{\partial U(p)}{\partial p}\right|_{p=\hat{p}[T]-\hat{p}_{\mathrm{EMA}}[T]} \\
& +\Delta \hat{\xi}[T-1]+\hat{\zeta}[T-1]
\end{aligned}
$$

under a random potential $U(p)=-c e^{-1 / \tau_{\mathrm{EMA}}} \Delta p^{*} Z_{\mathrm{EMA}} \hat{\tau}$ $[T-1] \log \left\{\cosh \left(e^{1 / \tau_{\mathrm{EMA}}} p / \Delta p^{*} Z_{\mathrm{EMA}}\right)\right\}$. In this sense, our theory is applicable in a straightforward manner to a derivation of the PUCK model.

\section{Possible generalization 2: Reduction to the random multiplicative processes}

In Sec. VIC, we assume $\Delta \tilde{p}^{*} \lesssim 1$ both for analytical simplicity and for consistency with the empirical report [46]. Here we discuss the case with $\Delta \tilde{p}^{*} \gg 1$, whereby the hyperbolic trend following reduces to the linear trend following as $c \tanh \left(\Delta \hat{p} / \Delta p^{*}\right) \approx c \Delta \hat{p} / \Delta p^{*}$. The financial Langevin equation (80) is thus replaced with a linear financial Langevin equation

$$
\Delta \hat{p}[T+1]=c \hat{\tau}[T] \frac{\Delta \hat{p}[T]}{\Delta p^{*}}+\Delta \hat{\xi}[T]+\hat{\zeta}[T] .
$$

By introducing the second-order difference $\Delta^{2} \hat{p}[T] \equiv$ $\Delta \hat{p}[T+1]-\Delta \hat{p}[T]$, we obtain a similar equation to the conventional Langevin equation as

$$
\Delta^{2} \hat{p}[T]=-\hat{\gamma}[T] \Delta \hat{p}[T]+\Delta \hat{\xi}[T]+\hat{\zeta}[T]
$$

with a random frictional coefficient $\hat{\gamma}[T] \equiv 1-c \hat{\tau}[T] / \Delta p^{*}$, consistently with the simplified discussion in the Supplemental Material of Ref. [46]. Since Eq. (113) belongs to the random multiplicative processes [58], the price movement obeys the power-law statistics, consistently with the previous exact solution [36] for the two-body case $N=2$.

\section{CONCLUSION}

In this paper, we have presented a systematic solution for the trend-following trader model, which was empirically introduced in our previous work [46]. Starting from the microscopic dynamics of individual traders, we have systematically reduced the multiagent dynamics by generalizing the mathematical method developed in molecular kinetic theory. We first introduce the phase space for our model and derive the dynamical equation for the phase space distribution function, which corresponds to the Liouville equation in conventional analytical mechanics. On the basis of the Liouville equation for the trend-following trader model, we derive a hierarchy 
of reduced distributions in a parallel method to the BBGKY hierarchy. By introducing the mean-filed approximation, corresponding to the assumption of molecular chaos, we derive the mean-field dynamical equation for the one-body distribution function, similarly to the Boltzmann equation. We then derive the analytic solution for the mean-field model, whose validity is numerically examined when the number of traders is sufficiently large. We also derive the financial Langevin equation, governing the macroscopic dynamics of the financial Brownian motion, and study the macroscopic properties of the market price movements. Our formulation clarifies the explicit mathematical correspondence between colloidal Brownian particle (water molecule) and market price (order book), as the physical picture for the financial Brownian motion heuristically proposed in Ref. [45].

Here we have clarified the power of the kinetic frameworks in describing financial markets from microscopic dynamics. In our conjecture, this success lies in the fact that the financial markets approximately satisfy the key assumptions of the binary interaction and molecular chaos; the one-to-one transaction (i.e., the binary interaction) is the most basic interaction and traders are less likely to transact with the same counterpart for large $N$. We believe that the financial market is one of the best areas to apply kinetic theory, besides traffic flow and wealth distribution [7-9,12]. We also believe that generalization of kinetic theories could be a key to clarifying various social systems from microscopic dynamics, since access to miscellaneous microscopic data is currently widely available.

In this paper, we studied the EBS interbank FX market, whose characters may be different from other financial markets (e.g., stock markets and retail FX markets). While most of the traders in our data set would be professional institutional investors, the presence of retail traders would not be negligible in general financial markets. Also, the strict two-way quote rule imposed on the key liquidity providers might be specific to the EBS market. It may be interesting to apply our analyses to other financial markets, to highlight their differences to the EBS market.

It would be also interesting to generalize our formulation for various traders employing different trading strategies. While we focused on the trend-following strategy, market makers apply various strategies, such as classical inventory management [i.e., they tend to buy (sell) the currency after selling (buying) to close their position]. The impact of introducing a variety of strategies would need to be pursued as a future topic.

\section{ACKNOWLEDGMENTS}

We acknowledge T. Ito, M. Katori, H. Hayakawa, M. Oshikawa, F. van Wijland, S. Ogawa, K. Yamada, T. Ooshida, D. Yanagisawa, S. Ichiki, K. Tamura, and J. Ozaki for fruitful discussions. We also appreciate NEX for providing us with the EBS data on their foreign exchange platform. This work was supported by the Japan Society for the Promotion of Science KAKENHI (Grants No. 16K16016 and No. 17J10781), the JST Strategic International Collaborative Research Program (SICORP) on the topic of "ICT for a Resilient Society" by Japan and Israel, the Joint Collaborative Research Laboratory for MUFG AI Financial Market Analysis, and a Challenging



FIG. 17. Qualitative picture of the NLO solution (61) depending on the regimes $\boldsymbol{R}_{0}, \boldsymbol{R}_{1}$, and $\boldsymbol{R}_{2} \equiv \boldsymbol{R}_{2}^{-} \cup \boldsymbol{R}_{2}^{+}$. The asymptotic behavior is described by Eq. (A2). The regimes $\boldsymbol{R}_{0}, \boldsymbol{R}_{1}$, and $\boldsymbol{R}_{2}$ represent the peak, transient, and tail regimes, respectively.

Research President's Honorary Award from the Tokyo Institute of Technology.

\section{APPENDIX A: NEXT-TO-LEADING-ORDER SOLUTION TO THE FINANCIAL BOLTZMANN EQUATION}

Here we check that the NLO solution (61) is the solution of the financial Boltzmann equation (51) by direct substitution. Before the detailed calculation, let us introduce several useful concepts and equations. We first introduce three regimes for the solution (61) (see Fig. 17 for the schematic):

(1) Around the peak: $\boldsymbol{R}_{0} \equiv(-\varepsilon / 2, \varepsilon / 2)$.

(2) Transient regime: $\boldsymbol{R}_{1} \equiv(-L / 2+\varepsilon / 2,-\varepsilon / 2) \cup$ $(\varepsilon / 2, L / 2-\varepsilon / 2)$.

(3) Around the tail: $\boldsymbol{R}_{2} \equiv \boldsymbol{R}_{2}^{-} \cup \boldsymbol{R}_{2}^{+} \quad$ with $\quad \boldsymbol{R}_{2}^{-} \equiv$ $(-\infty,-L / 2+\varepsilon / 2)$ and $\boldsymbol{R}_{2}^{+} \equiv(L / 2-\varepsilon / 2, \infty)$.

The tail regime $\boldsymbol{R}_{2}$ is rounded by the finite $N$ effect, whereby prices go beyond the c.m. with low but finite probability. Most of the transactions will occur in this regime and the newly submitted orders will return back near the peak $\boldsymbol{R}_{0}$. The peak regime $\boldsymbol{R}_{0}$ is also rounded because the newly submitted midprices return back from the tail regime $\boldsymbol{R}_{2}$. The boundary-layer thickness is given by $\varepsilon$ for both regimes. For the transient regime $\boldsymbol{R}_{\mathbf{1}}$, nothing will happen with high probability since it is far from the tails and peak.

We next summarize several useful relations. For $\mathcal{F}(r) \equiv$ $e^{-r^{2} / 2} / \sqrt{2 \pi}-(r / 2) \operatorname{erfc}(r / \sqrt{2})$, asymptotic relations hold:

$$
\begin{aligned}
& \mathcal{F}(r) \simeq \frac{1}{r^{2}} \frac{e^{-r^{2} / 2}}{\sqrt{2 \pi}} \quad(r \rightarrow+\infty), \\
& \mathcal{F}(r) \simeq-r \quad(r \rightarrow-\infty) .
\end{aligned}
$$

There is a symmetry such that $\phi^{L}(r)=\phi^{L}(-r)$. The solution (61) therefore implies

$$
\phi^{L}(r) \simeq \begin{cases}\psi^{L}(r)-\frac{8 \varepsilon}{L^{2}} \mathcal{F}\left(\frac{|r|}{\varepsilon}\right) & \left(r \in \boldsymbol{R}_{0}\right), \\ \psi^{L}(r) & \left(r \in \boldsymbol{R}_{1}\right), \\ \frac{4 \varepsilon}{L^{2}} \mathcal{F}\left(\frac{|r|-L / 2}{\varepsilon}\right) & \left(r \in \boldsymbol{R}_{2}\right),\end{cases}
$$

with the tent function $\psi^{L}(r) \equiv\left(4 / L^{2}\right) \max \{L / 2-|r|, 0\}$. In addition, we obtain an identity

$$
\left.\left|\tilde{\partial}_{r r^{\prime}}\right|\left[\mathcal{F}(r) \mathcal{F}\left(-r^{\prime}\right)\right]\right|_{r=r^{\prime}}=\frac{d^{2}}{d r^{2}} \mathcal{F}(r)=\frac{e^{-r^{2} / 2}}{\sqrt{2 \pi}} .
$$


The steady probability flux $\left.J_{s}^{L L^{\prime}}(r) \equiv\left(\sigma^{2} / 2\right)\left|\tilde{\partial}_{r r^{\prime}}\right| \phi^{L}(r) \phi^{L^{\prime}}\left(r^{\prime}\right)\right|_{r-r^{\prime}=s\left(L+L^{\prime}\right) / 2}$ is thus rewritten as

$$
\begin{aligned}
& J_{s=+1}^{L L^{\prime}}(r) \simeq \begin{cases}\frac{\sigma^{2}}{2} \frac{16 \varepsilon}{L^{2} L^{2}} \frac{1}{\sqrt{2 \pi}} e^{-(r-L / 2)^{2} / 2 \varepsilon^{2}} & \left(r \in \boldsymbol{R}_{2}^{+}\right), \\
0 & \left(r \notin \boldsymbol{R}_{2}^{+}\right),\end{cases} \\
& J_{s=-1}^{L L^{\prime}}(r) \simeq \begin{cases}\frac{\sigma^{2}}{2} \frac{16 \varepsilon}{L^{2} L^{2}} \frac{1}{\sqrt{2 \pi}} e^{-(r+L / 2)^{2} / 2 \varepsilon^{2}} & \left(r \in \boldsymbol{R}_{2}^{-}\right), \\
0 & \left(r \notin \boldsymbol{R}_{2}^{-}\right),\end{cases}
\end{aligned}
$$

where we have ignored exponentially small contributions. We note that the NLO solution (61) perturbatively satisfies the renormalization condition as

$$
\int_{-\infty}^{\infty} d r \phi^{L}(r) \simeq 1-\frac{1}{2}\left(1+\frac{4 \varepsilon^{2}}{L^{2}}\right) \operatorname{erfc}\left(\frac{L}{2 \sqrt{2} \varepsilon}\right)+\frac{2 \varepsilon}{L} \frac{e^{-L^{2} / 8 \varepsilon^{2}}}{\sqrt{2 \pi}}=1+o\left(\frac{\varepsilon}{L} \frac{e^{-L^{2} / 8 \varepsilon^{2}}}{\sqrt{2 \pi}}\right),
$$

which implies that Eq. (61) is the appropriate renormalized solution if it satisfies Eq. (51). ${ }^{9}$

Using the above relations, we next check that the NLO solution (61) satisfies Eq. (51) by direct substitution. For $r \in \boldsymbol{R}_{1}$, Eq. (61) obviously satisfies the financial Boltzmann equation (51), by considering the asymptotic relations (A2) and (A4). For $r \in \boldsymbol{R}_{2}^{+}$, we obtain

$$
\begin{aligned}
& \frac{\sigma^{2}}{2} \frac{\partial \phi^{L}}{\partial r^{2}}+N \sum_{s= \pm 1} \int_{L_{\min }}^{L_{\max }} d L^{\prime} \rho_{L^{\prime}}\left[J_{s}^{L L^{\prime}}(r+s L / 2)-J_{s}^{L L^{\prime}}(r)\right] \\
& \quad \simeq \frac{\sigma^{2}}{2} \frac{\partial \phi^{L}}{\partial r^{2}}-N \int_{L_{\min }}^{L_{\max }} d L^{\prime} \rho_{L^{\prime}} J_{s=+1}^{L L^{\prime}}(r)=\frac{\sigma^{2}}{2} \frac{4}{L^{2} \varepsilon} \frac{e^{-(r-L / 2)^{2} / 2 \varepsilon^{2}}}{\sqrt{2 \pi}}\left[1-4 \varepsilon^{2} N \int_{L_{\min }}^{L_{\max }} \frac{d L^{\prime} \rho_{L^{\prime}}}{L^{2}}\right]=0 .
\end{aligned}
$$

This implies that the NLO solution (61) asymptotically satisfies Eq. (51) for $r \in \boldsymbol{R}_{2}^{+}$. In a parallel calculation, we can also show that the NLO solution (61) satisfies Eq. (51) for $r \in \boldsymbol{R}_{2}^{-}$. For $r \in \boldsymbol{R}_{0}$, we obtain

$$
\begin{aligned}
& \frac{\sigma^{2}}{2} \frac{\partial \phi^{L}}{\partial r^{2}}+N \sum_{s= \pm 1} \int_{L_{\min }}^{L_{\max }} d L^{\prime} \rho_{L^{\prime}}\left[J_{s}^{L L^{\prime}}(r+s L / 2)-J_{s}^{L L^{\prime}}(r)\right] \\
& \quad \simeq \frac{\sigma^{2}}{2} \frac{\partial \phi^{L}}{\partial r^{2}}+N \sum_{s= \pm 1} \int_{L_{\min }}^{L_{\max }} d L^{\prime} \rho_{L^{\prime}} J_{s}^{L L^{\prime}}(r+s L / 2)=\frac{\sigma^{2}}{2}\left[-\frac{8}{\varepsilon L^{2}}+\frac{32 N \varepsilon}{L^{2}} \int_{L_{\min }}^{L_{\max }} \frac{d L^{\prime} \rho_{L^{\prime}}}{L^{\prime 2}}\right] \frac{e^{-r^{2} / 2 \varepsilon^{2}}}{\sqrt{2 \pi}}=0
\end{aligned}
$$

which implies that Eq. (61) satisfies Eq. (51) for $r \in \boldsymbol{R}_{0}$. In summary, the NLO asymptotic solution (61) is shown to satisfy the financial Boltzmann equation (51) globally.

\section{APPENDIX B: CONSISTENCY OF THE SOLUTION (61) WITH THE FINANCIAL BBGKY HIERARCHY (59)}

We have obtained the NLO solution (61) to the financial Boltzmann equation (51). The financial Boltzmann equation (51) is derived from the financial BBGKY hierarchal equation (40) with the three-body correlation terms assumed to be irrelevant in Sec. V A. Here we check that the consistency between the NLO solution (61) and this assumption. Let us introduce the three-body distribution function $\phi_{T}^{L L^{\prime} L^{\prime \prime}}\left(r, r^{\prime}, r^{\prime \prime}\right)$ for the relative prices as

$$
\phi_{t}^{L_{i} L_{j} L_{k}}\left(r, r^{\prime}, r^{\prime \prime}\right)=P_{t}^{i j k}\left(r, r^{\prime}, r^{\prime \prime}\right)
$$

Let us check that the following remaining term $R$ can be ignored for $N \rightarrow \infty$,

$$
\left.R \equiv N^{2} \int d r^{\prime} d L^{\prime} d L^{\prime \prime} \rho_{L^{\prime}} \rho_{L^{\prime \prime}}\left[\left|\tilde{\partial}_{r^{\prime} r^{\prime \prime}}\right| \phi_{t}^{L L^{\prime} L^{\prime \prime}}\left(r-\frac{s\left(L^{\prime}-L^{\prime \prime}\right)}{2 N}, r^{\prime}, r^{\prime \prime}\right)-\left|\tilde{\partial}_{r^{\prime} r^{\prime \prime}}\right| \phi_{t}^{L L^{\prime} L^{\prime \prime}}\left(r, r^{\prime}, r^{\prime \prime}\right)\right]\right|_{r^{\prime}-r^{\prime \prime}=s\left(L^{\prime}+L^{\prime \prime}\right) / 2},
$$

by assuming molecular chaos for the three-body distribution

$$
\phi_{t}^{L L^{\prime} L^{\prime \prime}}\left(r, r^{\prime}, r^{\prime \prime}\right) \approx \phi_{t}^{L}(r) \phi_{t}^{L^{\prime}}\left(r^{\prime}\right) \phi_{t}^{L^{\prime \prime}}\left(r^{\prime \prime}\right)
$$

Indeed, we obtain

$$
\left.R \approx N^{2} \sum_{s= \pm 1} \int d L^{\prime} d L^{\prime \prime} \rho_{L^{\prime}} \rho_{L^{\prime \prime}} \int d r^{\prime}\left|\tilde{\partial}_{r^{\prime} r^{\prime \prime}}\right| \phi_{t}^{L^{\prime}}\left(r^{\prime}\right) \phi_{t}^{L^{\prime \prime}}\left(r^{\prime \prime}\right)\right|_{r^{\prime}-r^{\prime \prime}=s\left(L^{\prime}+L^{\prime \prime}\right) / 2}\left[\phi_{t}^{L}\left(r-\frac{s\left(L^{\prime}-L^{\prime \prime}\right)}{2 N}\right)-\phi_{t}^{L}(r)\right] .
$$

\footnotetext{
${ }^{9}$ The uniqueness of the solution is technically assumed. The proofs of such mathematical details are out of the scope of this paper.
} 
By substituting the NLO solution (61), we obtain a relation

$$
\left.\int d r^{\prime}\left|\tilde{\partial}_{r^{\prime} r^{\prime \prime}}\right| \phi_{t}^{L^{\prime}}\left(r^{\prime}\right) \phi_{t}^{L^{\prime \prime}}\left(r^{\prime \prime}\right)\right|_{r^{\prime}-r^{\prime \prime}=s\left(L^{\prime}+L^{\prime \prime}\right) / 2} \approx \frac{4 L_{\rho}^{* 2}}{N L^{\prime 2} L^{\prime 2}} .
$$

$R$ is then shown to be of order $O\left(N^{-1}\right)$ as

$$
R \approx \sum_{k=1}^{\infty} \frac{4 L_{\rho}^{* 2}}{(2 k) !(2 N)^{2 k-1}} \int \frac{d L^{\prime} d L^{\prime \prime} \rho_{L^{\prime}} \rho_{L^{\prime \prime}}\left(L^{\prime}-L^{\prime \prime}\right)^{2 k}}{L^{\prime 2} L^{\prime 2}} \frac{\partial^{2 k} \phi_{t}^{L}(r)}{\partial r^{2 k}}=O\left(N^{-1}\right),
$$

where we have used the Taylor expansion

$$
\phi_{t}^{L}\left(r-\frac{s\left(L^{\prime}-L^{\prime \prime}\right)}{2 N}\right)-\phi_{t}^{L}(r)=\sum_{k=1}^{\infty} \frac{(-s)^{k}}{k !}\left(\frac{L^{\prime}-L^{\prime \prime}}{2 N}\right)^{k} \frac{\partial^{k} \phi_{t}^{L}(r)}{\partial r^{k}} .
$$

The neglect of the three-body correlation term $R$ is thus validated on the basis of the NLO solution (61).

\section{APPENDIX C: PROOF OF PROBABILITY CONSERVATION}

We here prove the conservation of the probability (64) under the reflecting boundary condition. The total probability where the order exists in the range $\left[-L_{\text {cut }} / 2, L_{\text {cut }} / 2\right]$ is given by $\int_{-L_{\text {cut }} / 2}^{L_{\text {cut }} / 2} d r \phi_{t}^{L}(r)$. The time derivative of the total probability obeys the following equation:

$$
\begin{aligned}
\frac{\partial}{\partial t} \int_{-L_{\text {cut }} / 2}^{L_{\text {cut }} / 2} d r \phi_{t}^{L}(r) & =\int_{-L_{\text {cut }} / 2}^{L_{\text {cut }} / 2} d r\left\{\frac{\sigma^{2}}{2} \frac{\partial^{2} \phi_{t}^{L}(r)}{\partial r^{2}}+N \sum_{s= \pm 1} \int_{L_{\min }}^{L_{\max }} d L^{\prime} \rho_{L^{\prime}}\left[J_{t ; s}^{L L^{\prime}}(r+s L / 2)-J_{t ; s}^{L L^{\prime}}(r)\right]\right\} \\
& =\frac{\sigma^{2}}{2}\left[\frac{\partial \phi_{t}^{L}(r)}{\partial r}\right]_{-L_{\text {cut }} / 2}^{L_{\text {cut }} / 2}+N \sum_{s= \pm 1} \int_{-L_{\text {cut }} / 2}^{L_{\text {cut }} / 2} d r \int_{L_{\min }}^{L_{\max }} d L^{\prime} \rho_{L^{\prime}}\left[J_{t ; s}^{L L^{\prime}}(r+s L / 2)-J_{t ; s}^{L L^{\prime}}(r)\right] .
\end{aligned}
$$

Considering the following identity for the integrals

$$
\begin{aligned}
\int_{-L_{\mathrm{cut}} / 2}^{L_{\mathrm{cut} / 2}} d r J_{t ; s=+1}^{L L^{\prime}}(r+s L / 2) & =\frac{\sigma^{2}}{2} \int_{-L_{\mathrm{cut}} / 2}^{L_{\mathrm{cut}} / 2} d r\left[\phi_{t}^{L^{\prime}}\left(r-L^{\prime} / 2\right)\left|\partial \phi_{t}^{L}(r+L / 2)\right|+\left|\partial \phi_{t}^{L^{\prime}}\left(r-L^{\prime} / 2\right)\right| \phi_{t}^{L}(r+L / 2)\right] \\
& =\frac{\sigma^{2}}{2} \int_{-\left(L_{\mathrm{cut}}-L^{\prime}\right) / 2}^{\left(L_{\mathrm{cut}}-L\right) / 2} d r\left[\phi_{t}^{L^{\prime}}\left(r-L^{\prime} / 2\right)\left|\partial \phi_{t}^{L}(r+L / 2)\right|+\left|\partial \phi_{t}^{L^{\prime}}\left(r-L^{\prime} / 2\right)\right| \phi_{t}^{L}(r+L / 2)\right]
\end{aligned}
$$

and

$$
\begin{aligned}
\int_{-L_{\mathrm{cut}} / 2}^{L_{\mathrm{cut}} / 2} d r J_{t ; s=+1}^{L L^{\prime}}(r) & =\frac{\sigma^{2}}{2} \int_{-L_{\mathrm{cut}} / 2}^{L_{\mathrm{cut} / 2}} d r\left[\phi_{t}^{L^{\prime}}\left(r-\left(L+L^{\prime}\right) / 2\right)\left|\partial \phi_{t}^{L}(r)\right|+\left|\partial \phi_{t}^{L^{\prime}}\left(r-\left(L+L^{\prime}\right) / 2\right)\right| \phi_{t}^{L}(r)\right] \\
& =\frac{\sigma^{2}}{2} \int_{\left(-L_{\mathrm{cut}}+L+L^{\prime}\right) / 2}^{L_{\mathrm{cut}} / 2} d r\left[\phi_{t}^{L^{\prime}}\left(r-\left(L+L^{\prime}\right) / 2\right)\left|\partial \phi_{t}^{L}(r)\right|+\left|\partial \phi_{t}^{L^{\prime}}\left(r-\left(L+L^{\prime}\right) / 2\right)\right| \phi_{t}^{L}(r)\right] \\
& =\frac{\sigma^{2}}{2} \int_{-\left(L_{\mathrm{cut}}-L^{\prime}\right) / 2}^{\left(L_{\mathrm{cut}}-L\right) / 2} d r\left[\phi_{t}^{L^{\prime}}\left(r-L^{\prime} / 2\right)\left|\partial \phi_{t}^{L}(r+L / 2)\right|+\left|\partial \phi_{t}^{L^{\prime}}\left(r-L^{\prime} / 2\right)\right| \phi_{t}^{L}(r+L / 2)\right],
\end{aligned}
$$

we obtain

$$
\int_{-L_{\mathrm{cut}} / 2}^{L_{\mathrm{cut}} / 2} d r\left[J_{t ; s=+1}^{L L^{\prime}}(r+L / 2)-J_{t ; s=+1}^{L L^{\prime}}(r)\right]=0 .
$$

Here, the assumption $L_{\text {cut }}>L_{\max }$ is used in changing the integral interval. In a parallel calculation, we obtain

$$
\int_{-L_{\text {cut }} / 2}^{L_{\text {cut }} / 2} d r\left[J_{t ; s=-1}^{L L^{\prime}}(r-L / 2)-J_{t ; s=-1}^{L L^{\prime}}(r)\right]=0 .
$$

These relations imply

$$
\frac{\partial}{\partial t} \int_{-L_{\mathrm{cut}} / 2}^{L_{\mathrm{cut}} / 2} d r \phi_{t}^{L}(r)=\frac{\sigma^{2}}{2}\left[\frac{\partial \phi_{t}^{L}(r)}{\partial r}\right]_{-L_{\mathrm{cut}} / 2}^{L_{\mathrm{cut}} / 2} .
$$

We then show the conservation of the probability (64) under the reflecting boundary condition. 
[1] R. Kubo, M. Toda, and N. Hashitsume, Statsitical Physics II (Springer-Verlag, Berlin, 1991), 2nd ed.

[2] S. Chapman and T. G. Cowling, The Mathematical Theory of Non-Uniform Gases (Cambridge University Press, Cambridge, 1970).

[3] C. Van den Broeck, R. Kawai, and P. Meurs, Phys. Rev. Lett. 93, 090601 (2004).

[4] C. Van den Broeck and R. Kawai, Phys. Rev. Lett. 96, 210601 (2006).

[5] N. V. Brilliantov and T. Pöschel, Kinetic Theory of Granular Gases (Oxford University Press, New York, 2004).

[6] E. Bertin, M. Droz, and G. Grégoire, Phys. Rev. E 74, 022101 (2006); J. Phys. A 42, 445001 (2009).

[7] D. Helbing, Rev. Mod. Phys. 73, 1067 (2001).

[8] A. Schadschneider, D. Chowdhury, and K. Nishinari, Stochastic Transport in Complex Systems: From Molecules to Vehicles (Elsevier, Amsterdam, 2010).

[9] I. Prigogine and R. Herman, Kinetic Theory of Vehicular Traffic (American Elsevier, New York, 1971).

[10] D. Cai, L. Tao, M. Shelley, and D. W. McLaughlin, Proc. Natl. Acad. Sci. USA 101, 7757 (2004).

[11] M. A. Buice and C. C. Chow, PLoS Comput. Biol. 9, e1002872 (2013).

[12] L. Pareschi and G. Toscani, Interacting Multiagent Systems (Oxford University Press, Oxford, 2014).

[13] C. W. Gardiner, Handbook of Stochastic Methods, 4th ed. (Springer, Berlin, 2009).

[14] P. Résibois and M. de Leener, Classical Kinetic Theory of Fluids (Wiley, New York, 1977).

[15] J.-P. Hansen and I. McDonald, Theory of Simple Liquids, 3rd ed. (Academic Press, Amsterdam, 2006).

[16] N. G. van Kampen, Stochastic Processes in Physics and Chemistry, 3rd ed. (Elsevier, Amsterdam, 2007); Can. J. Phys. 39, 551 (1961).

[17] H. Spohn, Rev. Mod. Phys. 52, 569 (1980).

[18] R. N. Mantegna and H. E. Stanley, Introduction to Econophysics: Correlations and Complexity in Finance (Cambridge University Press, Cambridge, 1999).

[19] F. Slanina, Essentials of Econophysics Modelling (Oxford University Press, Oxford, 2014).

[20] L. Bachelier, Annales Scientifiques de l'École Normale Supérieure 17, 21 (1900).

[21] A. Einstein, Ann. Phys. (Berlin) 322, 549 (1905).

[22] R. N. Mantegna and H. E. Stanley, Nature (London) 376, 46 (1995).

[23] T. Lux, Appl. Financ. Econ. 6, 463 (1996).

[24] V. Plerou, P. Gopikrishnan, L. A. Nunes Amaral, M. Meyer, and H. E. Stanley, Phys. Rev. E 60, 6519 (1999).

[25] D. M. Guillaume, M. M. Dacorogna, R. R. Davé, U. A. Müller, R. B. Olsen, and O. V. Pictet, Finance Stoch. 1, 95 (1997).

[26] F. M. Longin, J. Business 69, 383 (1996).

[27] J. D. Hamilton, Time Series Analysis (Princeton University Press, Princeton, 1994).

[28] R. F. Engle, Econometrica 50, 987 (1982).

[29] M. Takayasu, T. Mizuno, and H. Takayasu, Physica A 370, 91 (2006).

[30] A. S. Kyle, Econometrica 53, 1315 (1985).

[31] H. Takayasu, H. Miura, T. Hirabayashi, and K. Hamada, Physica A 184, 127 (1992).
[32] P. Bak, M. Paczuski, and M. Shubik, Physica A 246, 430 (1997).

[33] T. Lux and M. Marchesi, Nature (London) 397, 498 (1999).

[34] A.-H. Sato and H. Takayasu, Physica A 250, 231 (1998).

[35] K. Yamada, H. Takayasu, and M. Takayasua, Physica A 382, 340 (2007).

[36] K. Yamada, H. Takayasu, T. Ito, and M. Takayasu, Phys. Rev. E 79, 051120 (2009).

[37] K. Yamada, H. Takayasu, and M. Takayasu, J. Phys.: Conf. Ser. 221, 012015 (2010).

[38] S. Maslov, Physica A 278, 571 (2000).

[39] M. G. Daniels, J. D. Farmer, L. Gillemot, G. Iori, and E. Smith, Phys. Rev. Lett. 90, 108102 (2003).

[40] E. Smith, J. D. Farmer, L. Gillemot, and S. Krishnamurthy, Quantitative Finance 3, 481 (2003).

[41] J.-P. Bouchaud, M. Mézard, and M. Potters, Quantitative Finance 2, 251 (2002).

[42] J. D. Farmer, P. Patelli, and I. Zovko, Proc. Natl. Acad. Sci. USA 102, 2254 (2005).

[43] B. Tóth, Y. Lempérière, C. Deremble, J. de Lataillade, J. Kockelkoren, and J.-P. Bouchaud, Phys. Rev. X 1, 021006 (2011).

[44] J. Donier, J. Bonart, I. Mastromatteo, and J.-P. Bouchaud, Quantitative Finance 15, 1109 (2015).

[45] Y. Yura, H. Takayasu, D. Sornette, and M. Takayasu, Phys. Rev. Lett. 112, 098703 (2014); Phys. Rev. E 92, 042811 (2015).

[46] K. Kanazawa, T. Sueshige, H. Takayasu, and M. Takayasu, Phys. Rev. Lett. 120, 138301 (2018).

[47] D. J. Evans and G. Morriss, Statistical Mechanics of Nonequilibrium Liquids, 2nd ed. (Cambridge University Press, Cambridge, 2008).

[48] M. H. Ernst, J. R. Dorfmann, W. R. Hoegy, and J. M. J. van Leeuwen, Physica 45, 127 (1969).

[49] H. van Beijeren and M. H. Ernst, J. Stat. Phys. 21, 125 (1979).

[50] K. Kanazawa, Statistical Mechanics for Athermal Fluctuation: Non-Gaussian Noise in Physics (Springer, Berlin, 2017).

[51] C. Cercignani, R. Illner, and M. Pulvirenti, The Mathematical Theory of Dilute Gases (Springer, New York, 1994).

[52] A. B. Schmidt, Ecology of the Modern Institutional Spot FX: The EBS Market in 2011 (January 12, 2012). Available at SSRN: https://ssrn.com/abstract=1984070.

[53] EBS Dealing Rules, Appendix, EBS Market (at the time of June 2016).

[54] H. Haken, Synergetics: Introduction and Advanced Topics (Springer, Berlin, 2004).

[55] H. A. David and H. N. Nagaraja, Order Statistics, 3rd ed. (Wiley, New York, 2003).

[56] M. Takayasu, H. Takayasu, and M. P. Okazaki, Empirical Science of Financial Fluctuations (Springer, Tokyo, 2002), pp.18-25.

[57] T. Sueshige, K. Kanazawa, H. Takayasu, and M. Takayasu, PLOS ONE (to be published).

[58] H. Takayasu, A.-H. Sato, and M. Takayasu, Phys. Rev. Lett. 79, 966 (1997).

[59] E. A. Novikov, Sov. Phys. JETP 20, 1290 (1965); W. Horsthemke and R. Lefever, Noise-Induced Transitions: Theory and Applications in Physics, Chemistry, and Biology (SpringerVerlag, Berlin, 1984); R. F. Fox, Phys. Rev. A 33, 467 (1986).

[60] H. P. Breuer and F. Petruccione, The Theory of Open Quantum Systems (Oxford University Press, Oxford, 2002). 